\title{
Measurements of Direct CP Violation, CPT Symmetry, and Other Parameters in the Neutral Kaon System
}

\author{
A. Alavi-Harati, ${ }^{12}$ T. Alexopoulos,${ }^{12}$ M. Arenton, ${ }^{11}$ K. Arisaka, ${ }^{2}$ S. Averitte, ${ }^{10}$ R.F. Barbosa,, , A.R. Barker, ${ }^{5}$ \\ M. Barrio, ${ }^{4}$ L. Bellantoni, ${ }^{7}$ A. Bellavance, ${ }^{9}$ J. Belz,${ }^{10}$ D.R. Bergman,${ }^{10}$ E. Blucher, ${ }^{4}$ G.J. Bock,${ }^{7}$ \\ C. Bown, ${ }^{4}$ S. Bright,${ }^{4}$ E. Cheu,${ }^{1}$ S. Childress,${ }^{7}$ R. Coleman, ${ }^{7}$ M.D. Corcoran,${ }^{9}$ G. Corti, ${ }^{11}$ B. Cox,${ }^{11}$ \\ A.R. Erwin, ${ }^{12}$ R. Ford,${ }^{7}$ A. Glazov,${ }^{4}$ A. Golossanov, ${ }^{11}$ G. Graham,${ }^{4}$ J. Graham ${ }^{4}$ E. Halkiadakis,${ }^{10}$ \\ J. Hamm, ${ }^{12}$ K. Hanagaki, ${ }^{8}$ S. Hidaka, ${ }^{8}$ Y.B. Hsiung, ${ }^{7}$ V. Jejer, ${ }^{11}$ D.A. Jensen,${ }^{7}$ R. Kessler, ${ }^{4}$ \\ H.G.E. Kobrak ${ }^{3}$ J. LaDue, ${ }^{5}$ A. Lath,${ }^{10}$ A. Ledovskoy, ${ }^{11}$ P.L. McBride, ${ }^{7}$ P. Mikelsons,${ }^{5}$ E. Monnier ${ }^{4}$, f \\ T. Nakaya, ${ }^{7}$ K.S. Nelson, ${ }^{11}$ H. Nguyen, ${ }^{7}$ V. O'Dell, ${ }^{7}$ R. Pordes,${ }^{7}$ V. Prasad,${ }^{4}$ X.R. Qi,${ }^{7}$ B. Quinn,${ }^{4}$ \\ E.J. Ramberg, ${ }^{7}$ R.E. Ray, ${ }^{7}$ A. Roodman, ${ }^{4}$ S. Schnetzer, ${ }^{10}$ K. Senyo, ${ }^{8}$ P. Shanahan, ${ }^{7}$ P.S. Shawhan, ${ }^{4}$ \\ J. Shields, ${ }^{11}$ W. Slater, ${ }^{2}$ N. Solomey,${ }^{4}$ S.V. Somalwar, ${ }^{10}$ R.L. Stone, ${ }^{10}$ E.C. Swallow, ${ }^{4,6}$ S.A. Taegar, ${ }^{1}$ \\ R.J. Tesarek, ${ }^{10}$ G.B. Thomson, ${ }^{10}$ P.A. Toale,${ }^{5}$ A. Tripathi, ${ }^{2}$ R. Tschirhart,${ }^{7}$ S.E. Turner,${ }^{2,4}$ Y.W. Wah, ${ }^{4}$ \\ J. Wang, ${ }^{1}$ H.B. White, ${ }^{7}$ J. Whitmore ${ }^{7}$ B. Winstein, ${ }^{4}$ R. Winston, ${ }^{4}$ T. Yamanaka, ${ }^{8}$ and E.D. Zimmerman ${ }^{4}$
}

\author{
(The $\mathrm{KTeV}$ Collaboration) \\ ${ }^{1}$ University of Arizona, Tucson, Arizona 85721 \\ ${ }^{2}$ University of California at Los Angeles, Los Angeles, California 90095 \\ ${ }^{3}$ University of California at San Diego, La Jolla, California 92093 \\ ${ }^{4}$ The Enrico Fermi Institute, The University of Chicago, Chicago, Illinois 60637 \\ ${ }^{5}$ University of Colorado, Boulder, Colorado 80309 \\ ${ }^{6}$ Elmhurst College, Elmhurst, Illinois 60126 \\ ${ }^{7}$ Fermi National Accelerator Laboratory, Batavia, Illinois 60510 \\ ${ }^{8}$ Osaka University, Toyonaka, Osaka 560-0043 Japan \\ ${ }^{9}$ Rice University, Houston, Texas 77005 \\ ${ }^{10}$ Rutgers University, Piscataway, New Jersey 08854 \\ ${ }^{11}$ The Department of Physics and Institute of Nuclear and Particle \\ Physics, University of Virginia, Charlottesville, Virginia 22901 \\ ${ }^{12}$ University of Wisconsin, Madison, Wisconsin 53706
}

(Dated: August 6, 2002)

\begin{abstract}
We present a series of measurements based on $K_{L, S} \rightarrow \pi^{+} \pi^{-}$and $K_{L, S} \rightarrow \pi^{0} \pi^{0}$ decays collected in 1996-1997 by the KTeV experiment (E832) at Fermilab. We compare these four $K \rightarrow \pi \pi$ decay rates to measure the direct $\mathrm{CP}$ violation parameter $R e\left(\epsilon^{\prime} / \epsilon\right)=(20.7 \pm 2.8) \times 10^{-4}$. We also test CPT symmetry by measuring the relative phase between the $\mathrm{CP}$ violating and $\mathrm{CP}$ conserving decay amplitudes for $K \rightarrow \pi^{+} \pi^{-}\left(\phi_{+-}\right)$and for $K \rightarrow \pi^{0} \pi^{0}\left(\phi_{00}\right)$. We find the difference between the relative phases to be $\Delta \phi \equiv \phi_{00}-\phi_{+-}=(+0.39 \pm 0.50)^{\circ}$, and the deviation of $\phi_{+-}$from the superweak phase to be $\phi_{+-}-\phi_{S W}=(+0.61 \pm 1.19)^{\circ}$; both results are consistent with CPT symmetry. In addition, we present new measurements of the $K_{L}-K_{S}$ mass difference and $K_{S}$ lifetime: $\Delta m=(5261 \pm 15) \times 10^{6} \hbar \mathrm{s}^{-1}$ and $\tau_{S}=(89.65 \pm 0.07) \times 10^{-12} \mathrm{~s}$.

PACS numbers: 11.30.Er, 13.25.Es, 14.40.Aq
\end{abstract}

\section{INTRODUCTION}

The discovery of the $K_{L} \rightarrow \pi^{+} \pi^{-}$decay in 1964 [1] demonstrated that CP symmetry is violated in weak interactions. Subsequent experiments showed that the effect is mostly due to a small asymmetry between the $K^{0} \rightarrow \overline{K^{0}}$ and $\overline{K^{0}} \longrightarrow K^{0}$ transition rates, which is referred to as indirect $\mathrm{CP}$ violation. Over the last three decades, significant effort has been devoted to searching for direct $\mathrm{CP}$ violation in a decay amplitude.

Direct $\mathrm{CP}$ violation can be detected by comparing the

\footnotetext{
*Permanent addressUniversity of São Paulo, São Paulo, Brazil

${ }^{\dagger}$ Permanent address C.P.P. Marseille/C.N.R.S., France
}

level of CP violation for different decay modes. The parameters $\epsilon$ and $\epsilon^{\prime}$ are related to the ratio of $\mathrm{CP}$ violating to $\mathrm{CP}$ conserving decay amplitudes for $K \rightarrow \pi^{+} \pi^{-}$and $K \rightarrow \pi^{0} \pi^{0}$ :

$$
\begin{aligned}
& \eta_{+-} \equiv \frac{A\left(K_{L} \rightarrow \pi^{+} \pi^{-}\right)}{A\left(K_{S} \rightarrow \pi^{+} \pi^{-}\right)}=\epsilon+\epsilon^{\prime}, \\
& \eta_{00} \equiv \frac{A\left(K_{L} \rightarrow \pi^{0} \pi^{0}\right)}{A\left(K_{S} \rightarrow \pi^{0} \pi^{0}\right)}=\epsilon-2 \epsilon^{\prime} .
\end{aligned}
$$

$\epsilon$ is a measure of indirect $\mathrm{CP}$ violation, which is common to all decay modes. If CPT symmetry holds, the phase of $\epsilon$ is equal to the "superweak" phase:

$$
\phi_{S W} \equiv \tan ^{-1}(2 \Delta m / \Delta \Gamma),
$$

where $\Delta m \equiv m_{L}-m_{S}$ is the $K_{L^{-}} K_{S}$ mass difference and $\Delta \Gamma=\Gamma_{S}-\Gamma_{L}$ is the difference in the decay widths. 
The quantity $\epsilon^{\prime}$ is a measure of direct $\mathrm{CP}$ violation, which contributes differently to the $\pi^{+} \pi^{-}$and $\pi^{0} \pi^{0}$ decay modes, and is proportional to the difference between the decay amplitudes for $K^{0} \rightarrow \pi^{+} \pi^{-}\left(\pi^{0} \pi^{0}\right)$ and $\overline{K^{0}} \rightarrow \pi^{+} \pi^{-}\left(\pi^{0} \pi^{0}\right)$. Measurements of $\pi \pi$ phase shifts [2] show that, in the absence of CPT violation, the phase of $\epsilon^{\prime}$ is approximately equal to that of $\epsilon$. Therefore, $\operatorname{Re}\left(\epsilon^{\prime} / \epsilon\right)$ is a measure of direct $\mathrm{CP}$ violation and $\operatorname{Im}\left(\epsilon^{\prime} / \epsilon\right)$ is a measure of CPT violation.

Experimentally, $\operatorname{Re}\left(\epsilon^{\prime} / \epsilon\right)$ is determined from the double ratio of the two pion decay rates of $K_{L}$ and $K_{S}$ :

$$
\begin{aligned}
\frac{\Gamma\left(K_{L} \rightarrow \pi^{+} \pi^{-}\right) / \Gamma\left(K_{S} \rightarrow \pi^{+} \pi^{-}\right)}{\Gamma\left(K_{L}\right.} & \left.\rightarrow \pi^{0} \pi^{0}\right) / \Gamma\left(K_{S} \rightarrow \pi^{0} \pi^{0}\right) \\
& =\left|\frac{\eta_{+-}}{\eta_{00}}\right|^{2} \approx 1+6 \operatorname{Re}\left(\epsilon^{\prime} / \epsilon\right) .
\end{aligned}
$$

For small $\left|\epsilon^{\prime} / \epsilon\right|, \operatorname{Im}\left(\epsilon^{\prime} / \epsilon\right)$ is related to the phases of $\eta_{+-}$ and $\eta_{00}$ by

$$
\Delta \phi \equiv \phi_{00}-\phi_{+-} \approx-3 \operatorname{Im}\left(\epsilon^{\prime} / \epsilon\right) .
$$

The Standard Model accommodates both direct and indirect $\mathrm{CP}$ violation 3 , 田, 5. Unfortunately, there are large hadronic uncertainties in the calculation of $\operatorname{Re}\left(\epsilon^{\prime} / \epsilon\right)$. Most recent Standard Model predictions for $\operatorname{Re}\left(\epsilon^{\prime} / \epsilon\right)$ are less than $30 \times 10^{-4}[6,7,8,9,10,11,12,13,14,15,16$. The superweak model [17], proposed shortly after the discovery of $K_{L} \rightarrow \pi^{+} \pi^{-}$, also accommodates indirect $\mathrm{CP}$ violation, but not direct $\mathrm{CP}$ violation.

Previous measurements have established that $\operatorname{Re}\left(\epsilon^{\prime} / \epsilon\right)$ is non-zero 18, 19, 20, 21. This paper reports an improved measurement of $R e\left(\epsilon^{\prime} / \epsilon\right)$ by the $\mathrm{KTeV}$ Experiment (E832) at Fermilab. This measurement is based on 40 million reconstructed $K \rightarrow \pi \pi$ decays collected in 1996 and 1997, and represents half of the total KTeV data sample. The $1996+1997$ sample is four times larger than, and contains, our previously published sample [20]. We also present measurements of the kaon parameters $\Delta m$ and $\tau_{S}$, and tests of CPT symmetry based on measurements of $\Delta \phi$ and $\phi_{+-}-\phi_{S W}$.

The outline of the paper is as follows. Section II describes the $\mathrm{KTeV}$ measurement technique, including details about the neutral hadron beams (Sec. IIB) and the detector used to identify $K \rightarrow \pi \pi$ decays (Sec. II d). The detector description also includes the calibration procedures and the performance characteristics relevant to the $\operatorname{Re}\left(\epsilon^{\prime} / \epsilon\right)$ measurement. The Monte Carlo simulation of the kaon beams and detector is described in Section IIE. Section III explains the reconstruction and event selection for the $K \rightarrow \pi^{+} \pi^{-}$and $K \rightarrow \pi^{0} \pi^{0}$ decay modes, and also the background subtraction for each mode. Section IV describes the acceptance correction, and the fit used to extract $\operatorname{Re}\left(\epsilon^{\prime} / \epsilon\right)$ and other physics parameters. Each of these sections is followed by a discussion of systematic uncertainties related to that part of the $\operatorname{Re}\left(\epsilon^{\prime} / \epsilon\right)$ measurement. Section $\mathrm{V}$ presents the $\operatorname{Re}\left(\epsilon^{\prime} / \epsilon\right)$ result along with several crosschecks. Finally,
Section VI presents our measurements of the kaon parameters $\Delta m, \tau_{S}, \Delta \phi$, and $\phi_{+-}$. Additional details of the work presented here are given in [22].

\section{MEASUREMENT TECHNIQUE AND APPARATUS}

\section{A. Overview}

The measurement of $\operatorname{Re}\left(\epsilon^{\prime} / \epsilon\right)$ requires a source of $K_{L}$ and $K_{S}$ decays, and a detector to reconstruct the charged $\left(\pi^{+} \pi^{-}\right)$and neutral $\left(\pi^{0} \pi^{0}\right)$ final states. The strategy of the $\mathrm{KTeV}$ experiment is to produce two identical $K_{L}$ beams, and then to pass one of the beams through a thick "regenerator." The beam that passes through the regenerator is called the regenerator beam, and the other beam is called the vacuum beam. The regenerator creates a coherent $\left|K_{L}\right\rangle+\rho\left|K_{S}\right\rangle$ state, where $\rho$ is the regeneration amplitude chosen such that most of the $K \rightarrow \pi \pi$ decay rate downstream of the regenerator is from the $K_{S}$ component. The measured quantities are the numbers of $K \rightarrow \pi^{+} \pi^{-}$and $K \rightarrow \pi^{0} \pi^{0}$ decays in the vacuum and regenerator beams. The vacuum-to-regenerator "single ratios" for $K \rightarrow \pi^{+} \pi^{-}$and $K \rightarrow \pi^{0} \pi^{0}$ decays are proportional to $\left|\eta_{+-} / \rho\right|^{2}$ and $\left|\eta_{00} / \rho\right|^{2}$, and the ratio of these two quantities gives $\operatorname{Re}\left(\epsilon^{\prime} / \epsilon\right)$ via Eq. 3 (also see Appendix A). The effect of $K_{S}-K_{L}$ interference in the regenerator beam is accounted for in a fitting program used to extract $\operatorname{Re}\left(\epsilon^{\prime} / \epsilon\right)$.

To reduce systematic uncertainties related to left-right asymmetries in the detector and beamline, the regenerator position alternates between the two beams once per minute. Decays in both beams are collected simultaneously to reduce sensitivity to time-dependent variations in the beam intensity and in detector efficiencies. The fixed geometry of the beamline elements, combined with alternating the regenerator position, ensures a constant vacuum-to-regenerator kaon flux ratio.

$K \rightarrow \pi^{+} \pi^{-}$decays are detected in a spectrometer consisting of four drift chambers and a dipole magnet; the well-known kaon mass is used to determine the momentum scale. The four photons from $K \rightarrow \pi^{0} \pi^{0}$ decays are detected in a pure Cesium Iodide (CsI) calorimeter; electrons from $K_{L} \rightarrow \pi^{ \pm} e^{\mp} \nu$ decays $\left(K_{e 3}\right)$ are used to calibrate the CsI energy scale. An extensive veto system is used to reject events coming from interactions in the regenerator, and to reduce backgrounds from kaon decays into non- $\pi \pi$ final states such as $K_{L} \rightarrow \pi^{ \pm} \mu^{\mp} \nu$ and $K_{L} \rightarrow \pi^{0} \pi^{0} \pi^{0}$.

A Monte Carlo simulation is used to correct for the acceptance difference between $K \rightarrow \pi \pi$ decays in the two beams, which results from the very different $K_{L}$ and $K_{S}$ lifetimes. The simulation includes details of detector geometry and efficiency, as well as the effects of "accidental" activity from the high flux of particles hitting the detector. The decay-vertex distributions provide a critical check of the simulation. To study the detector per- 


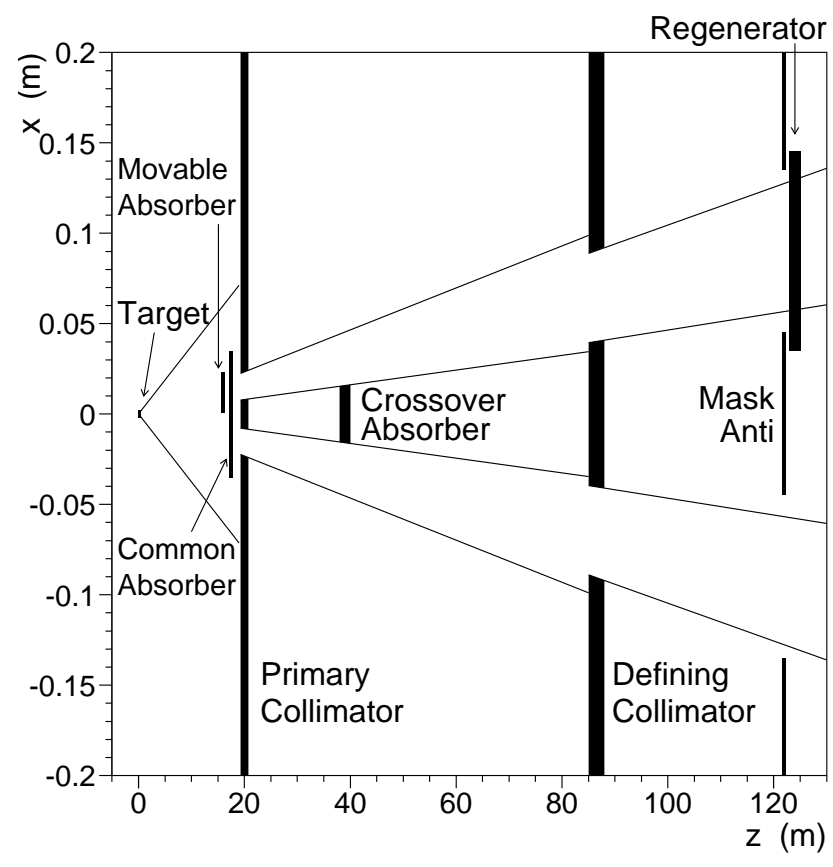

FIG. 1: Top view of the $\mathrm{KTeV}$ beamline, showing the $\mathrm{BeO}$ target, absorbers, collimating system, Mask Anti, and regenerator. The common absorber is a $7.62 \mathrm{~cm}$ thick $\mathrm{Pb}$ slab followed by a $52.3 \mathrm{~cm}$ slab of beryllium. The movable absorber is a $45.7 \mathrm{~cm}$ slab of beryllium that alternates beam positions once every minute along with the regenerator. The crossover absorber is a $2 \mathrm{~m}$ thick iron slab. Note the different horizontal and vertical scales.

formance, and to verify the accuracy of the Monte Carlo simulation, we also collect samples of $K_{L} \rightarrow \pi^{ \pm} e^{\mp} \nu$ and $K_{L} \rightarrow \pi^{0} \pi^{0} \pi^{0}$ decays with much higher statistics than the $K \rightarrow \pi \pi$ signal samples.

\section{B. The Kaon Beams}

Neutral kaons are produced by a proton beam hitting a fixed target (Fig. 11). The Fermilab Tevatron provides $3 \times 10^{12} 800 \mathrm{GeV} / c$ protons in a $20 \mathrm{~s}$ extraction cycle ("spill") once every minute. The proton beam has a $53 \mathrm{MHz}$ RF micro-structure such that protons arrive in $\sim 1$ ns wide "buckets" and at 19 ns intervals. The bucketto-bucket variations in beam intensity are typically $10 \%$.

The target is a narrow beryllium oxide $(\mathrm{BeO})$ rod, $3 \times$ $3 \mathrm{~mm}^{2}$ in the dimensions transverse to the beam, and $30 \mathrm{~cm}$ long corresponding to about one proton interaction length. The proton beam is incident on the target at a downward angle of $4.8 \mathrm{mrad}$ with respect to the line joining the target and the center of the detector. This targeting angle is chosen as a compromise between higher kaon flux at small angles, and a smaller neutron-to-kaon ratio $(n / K)$ at large angles.

The center of the $\mathrm{BeO}$ target defines the origin of the $\mathrm{KTeV}$ right-handed coordinate system. The positive $z$ axis is directed from the target to the center of the de- tector. The positive $y$-axis is directed up.

The particles produced in the $\mathrm{BeO}$ target include very few kaons compared to other hadrons and photons. A $\sim 100 \mathrm{~m}$ long beamline is used to remove unwanted particles and to collimate two well-defined kaon beams. In this beamline, charged particles are removed with sweeping magnets, photons are absorbed by a $7.62 \mathrm{~cm} \mathrm{~Pb} \mathrm{slab}$ located at $z=19 \mathrm{~m}$, and most of the hyperons decay near the target. To reduce the neutron-to-kaon ratio, neutrons (kaons) are attenuated by a factor of 4.6 (2.6) in a beryllium absorber that is common to both beams. An extra "movable" absorber, synchronized with the regenerator position, provides an additional attenuation factor of 3.8 (2.3) for neutrons (kaons) in the regenerator beam.

Each neutral kaon beam is defined by two collimators: a $1.5 \mathrm{~m}$ long primary collimator $(z=20 \mathrm{~m})$, and a $3 \mathrm{~m}$ long "defining" collimator $(z=85-88 \mathrm{~m})$ that defines the size and solid angle of each beam. Each collimator has two square holes, which are tapered to reduce scattering. A "crossover" absorber at $z=40 \mathrm{~m}$ prevents kaons that scatter in the upstream absorbers from crossing over into the other beam. At the defining collimator, the two beams have the same size $\left(4.4 \times 4.4 \mathrm{~cm}^{2}\right)$ and solid angle $(0.24 \mu \mathrm{str})$. The beam centers are separated by $14.2 \mathrm{~cm}$, and the horizontal angle between the two beams is $1.6 \mathrm{mrad}$.

The two beams pass through an evacuated volume, held at $10^{-6}$ Torr, which extends from $28 \mathrm{~m}$ to $159 \mathrm{~m}$ from the target. This evacuated region includes the $110 \mathrm{~m}$ to $158 \mathrm{~m}$ range used in the analysis. The downstream end of the evacuated volume is sealed with a $0.14 \%$ radiation length $\left(X_{0}\right)$ vacuum-window made of kevlar and mylar.

Most of the $K_{S}$ component decays near the $\mathrm{BeO}$ target. Downstream of the defining collimator, the small "target$K_{S}$ " component that remains in the vacuum beam increases the $K \rightarrow \pi \pi$ decay rate by $0.4 \%$ compared with a pure $K_{L}$ beam of equal intensity. This contribution from target- $K_{S}$ is essentially zero for kaon momenta below $100 \mathrm{GeV} / c$ and rises to $15 \%$ at $160 \mathrm{GeV} / c$.

In the regenerator beam, most of the $K \rightarrow \pi \pi$ decays are from the regenerated $K_{S}$ component. Decays from the $K_{L}$ component and from $K_{S}-K_{L}$ interference account for about $20 \%$ of the $K \rightarrow \pi \pi$ decay rate, ranging from $5 \%$ near the regenerator to $90 \%$ at the vacuum-window.

The composition of the neutral hadron beams is as follows. At $90 \mathrm{~m}$ from the $\mathrm{BeO}$ target, the vacuum beam has a $2.0 \mathrm{MHz}$ flux of kaons with $n / K=1.3$, and an average kaon momentum of about $70 \mathrm{GeV} / c$. Upstream of the regenerator, the kaon flux is $0.9 \mathrm{MHz}$ with $n / K=0.8$; downstream of the regenerator the flux of unscattered kaons is $0.15 \mathrm{Mhz}$, or 13 times smaller than in the vacuum beam. The flux of hyperons at $90 \mathrm{~m}$ from the target is about $1 \mathrm{kHz}$ in the vacuum beam. In addition to hadrons, there are muons that come from the $\mathrm{BeO}$ target, the proton beam dump, and kaon decays; the total muon flux hitting the $\mathrm{KTeV}$ detector is about $200 \mathrm{kHz}$. 


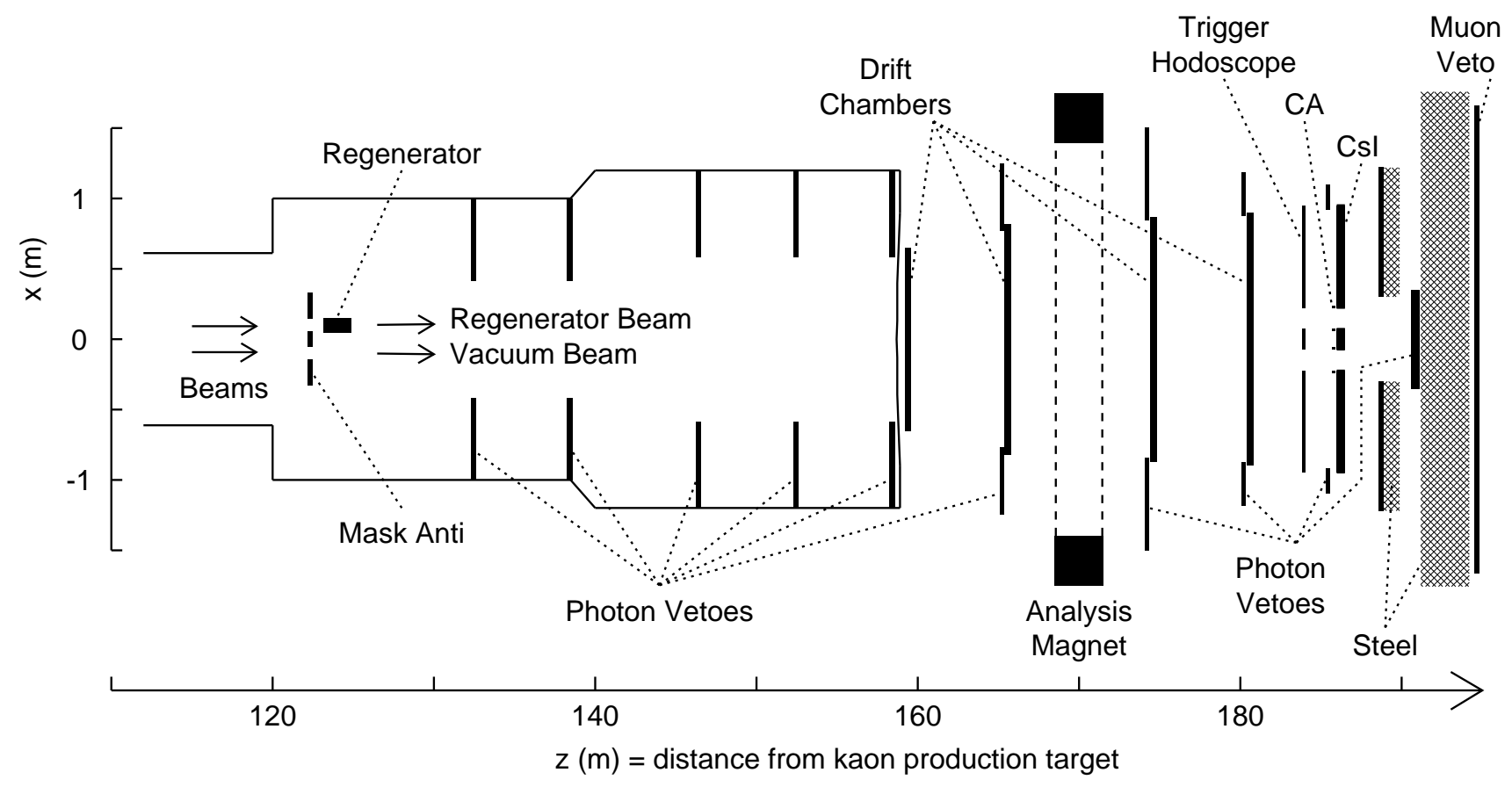

FIG. 2: Top view of the $\mathrm{KTeV}$ (E832) detector. The evacuated decay volume extends to $z=159 \mathrm{~m}$.

\section{The Detector}

Kaon decays downstream of the defining collimator $(z=85 \mathrm{~m}$, Fig. 1) are reconstructed by the KTeV detector (Fig. 2), which includes a magnetic spectrometer, CsI calorimeter, and veto system. Downstream of the vacuum-window, the space between detector components is filled with helium to reduce interactions from the neutral beam, and to reduce multiple scattering and photon conversions of decay products. The total amount of material upstream of the CsI calorimeter corresponds to $4 \%$ of a radiation length; about $60 \%$ of the material is in the trigger hodoscope just upstream of the CsI calorimeter, and $10 \%$ of the material is upstream of the first drift chamber. Each of the two neutral beams passes through holes in the Mask Anti veto, trigger hodoscope, and CsI calorimeter. The beams finally strike a beam-hole photon veto 5 meters downstream of the CsI. The following sections describe the detector components in more detail.

\section{Spectrometer}

The KTeV spectrometer includes four drift chambers (DCs) that measure charged particle positions. The two downstream chambers are separated from the upstream chambers by a $3 \times 2 \mathrm{~m}^{2}$ aperture dipole magnet. The magnet produces a field that is uniform to better than $1 \%$ and imparts a $0.41 \mathrm{GeV} / c$ kick in the horizontal plane. During data taking, the magnet polarity was reversed every 1-2 days.

The DC planes have a hexagonal cell geometry formed by six field-shaping wires surrounding each sense wire (Fig. 3). The cells are $6.35 \mathrm{~mm}$ wide, and the drift velocity is about $50 \mu \mathrm{m} / \mathrm{ns}$ in the $50-50$ argon-ethane gas mixture. The maximum drift time across a cell is $150 \mathrm{~ns}$, and defines the width of the "in-time" window. A chamber consists of two planes of horizontal wires to measure $y$ hit positions, and two planes of vertical wires to measure $x$ hit positions; the two $x$-planes, as well as the two $y$ planes, are offset by one half-cell to resolve the left-right ambiguity. The $x$ and $y$ hits cannot be associated to each other using only drift chamber information, but can be matched using CsI clusters as explained in Section III B.

There are a total of 16 planes and 1972 sense wires in the four DCs. The transverse chamber size increases with distance from the target. The smallest chamber (DC1) is $1.26 \times 1.26 \mathrm{~m}^{2}$ and has 101 sense wires per plane; the largest chamber (DC4) is $1.77 \times 1.77 \mathrm{~m}^{2}$ and has 140 sense wires per plane.

Lecroy 3373 multi-hit Time-to-Digital Converters (TDCs) are used to measure the drift times relative to the RF-synchronized Level 1 trigger. The TDC resolution is $0.25 \mathrm{~ns}$, which contributes $13 \mu \mathrm{m}$ to the position resolution. The total TDC time window is 2.5 times longer than the in-time window, and is centered on the in-time window. The track reconstruction software uses only the earliest in-time hit on each wire. Hits prior to the in-time window are recorded to study their influence on in-time hits.

Each measured drift time, $t$, is converted into a drift distance, $x$, with a non-linear $x(t)$ map. The maps are measured separately for each of the sixteen planes using the uniform hit-illumination across each cell. The $x(t)$ 


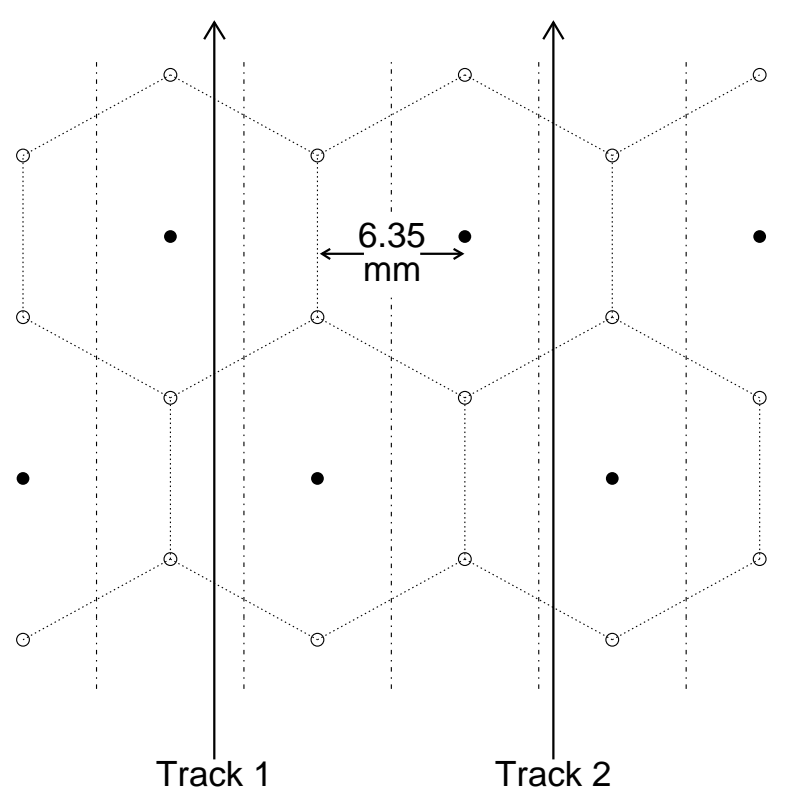

FIG. 3: Drift chamber hexagonal cell geometry showing six field wires (open circles) around each sense wire (solid dots). The vertical dashed lines denote the boundaries of the "offsetcells" used in the track-separation cut (see Section III B). For example, two offset-cells are between tracks 1 and 2 .

calibrations are performed in roughly 1-day time periods.

A charged-particle track produces a hit in each of the two $x$ and $y$ planes. The two $x$ hits (or two $y$ hits) in each plane are referred to as a "hit-pair." For a track that is perpendicular to a drift chamber with perfect resolution, the sum of drift distances (SOD) from each hit-pair would equal the cell width of $6.35 \mathrm{~mm}$. The measured SOD distribution is shown in Fig. 田. For inclined tracks, an angular correction is applied to the SOD. The trackfinding software requires the SOD to be within $1 \mathrm{~mm}$ of the cell width. The mean SOD is stable to within $10 \mu \mathrm{m}$ during the run. The single-hit position resolution is typically $110 \mu \mathrm{m}$, corresponding to a SOD resolution of $150 \mu \mathrm{m}$ and a hit-pair resolution of $80 \mu \mathrm{m}$. Using the tracking algorithm described in Section III B, the momentum resolution is $\sigma_{p} / p \simeq[1.7 \oplus(p / 14)] \times 10^{-3}$, where $p$ is the track momentum in $\mathrm{GeV} / c$.

The average inefficiency for reconstructing a hit-pair is $3.7 \%$. Delta rays and accidental hits contribute to the low-side tail of the SOD distribution (Fig. \), and result in a hit-pair loss of $0.5 \%$ and $0.7 \%$, respectively. The remaining $2.5 \%$ loss is from missing hits and from SOD values more than $1 \mathrm{~mm}$ larger than the cell size

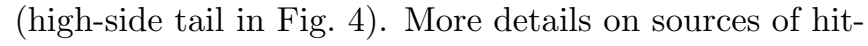
pair inefficiency are given in the description of the Monte Carlo simulation (Sec. II E 2).

To reconstruct the trajectories of charged particles accurately, the alignment of the drift chambers relative to each other, to the target, and to the CsI calorimeter must

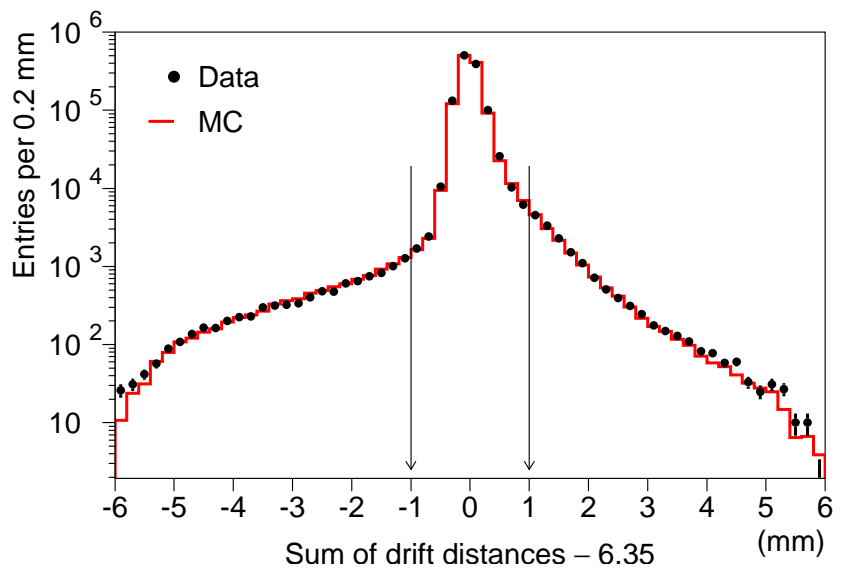

FIG. 4: Deviation of the sum of drift distances (SOD) from the nominal cell size of $6.35 \mathrm{~mm}$, for the $y$-views using a sample of $K \rightarrow \pi^{+} \pi^{-}$decays. The arrows indicate the $\pm 1 \mathrm{~mm}$ requirement for a good hit-pair. The data are shown as dots. The histogram is the Monte Carlo (MC) prediction (Sec. IIE 2).

be known. This alignment is determined in roughly 1-day periods using data samples described below.

The transverse alignment of the drift chambers is based on muons from dedicated runs with the analysis magnet turned off. The muon intensity is raised to $\sim 1 \mathrm{MHz}$ by reducing the field in the upstream sweeping magnets, and the neutral hadron beam is absorbed by a $2 \mathrm{~m}$ long steel block placed in the beam at $z=90 \mathrm{~m}$. These muon runs were performed every 1-2 days when the magnet polarity was reversed. Software calibration results in a transverse alignment of each $x$ and $y$ plane to within $10 \mu \mathrm{m}$, and relative rotations known to within $20 \mu \mathrm{rad}$.

The transverse target position is determined with a precision of $35 \mu \mathrm{m}$ using $K \rightarrow \pi^{+} \pi^{-}$decays in the vacuum beam and projecting the reconstructed kaon trajectory back to the target. The CsI offset relative to the drift chambers is measured using electrons from $K_{L} \rightarrow \pi^{ \pm} e^{\mp} \nu$ decays.

\section{The CsI Calorimeter}

The $\mathrm{KTeV}$ electromagnetic calorimeter consists of 3100 pure CsI crystals as shown in Fig. 5. There are $22322.5 \times$ $2.5 \mathrm{~cm}^{2}$ crystals in the central region, each viewed by a $1.9 \mathrm{~cm}$ Hamamatsu R5364 photomultiplier tube (PMT). There are also $8685 \times 5 \mathrm{~cm}^{2}$ crystals, each viewed by a $3.8 \mathrm{~cm}$ Hamamatsu R5330 PMT. The transverse size of the calorimeter is $1.9 \times 1.9 \mathrm{~m}^{2}$, and the length of each crystal is $50 \mathrm{~cm}\left(27 X_{0}\right)$. Two $15 \times 15 \mathrm{~cm}^{2}$ carbon fiber beam pipes allow the few $\mathrm{MHz}$ of beam particles to pass through the calorimeter without striking any material.

The crystals were individually wrapped and tested with a ${ }^{137} \mathrm{Cs}$ source to ensure that the response over the length of each crystal is uniform to within $\sim 5 \%$ [23]. This longitudinal uniformity requirement is necessary to 


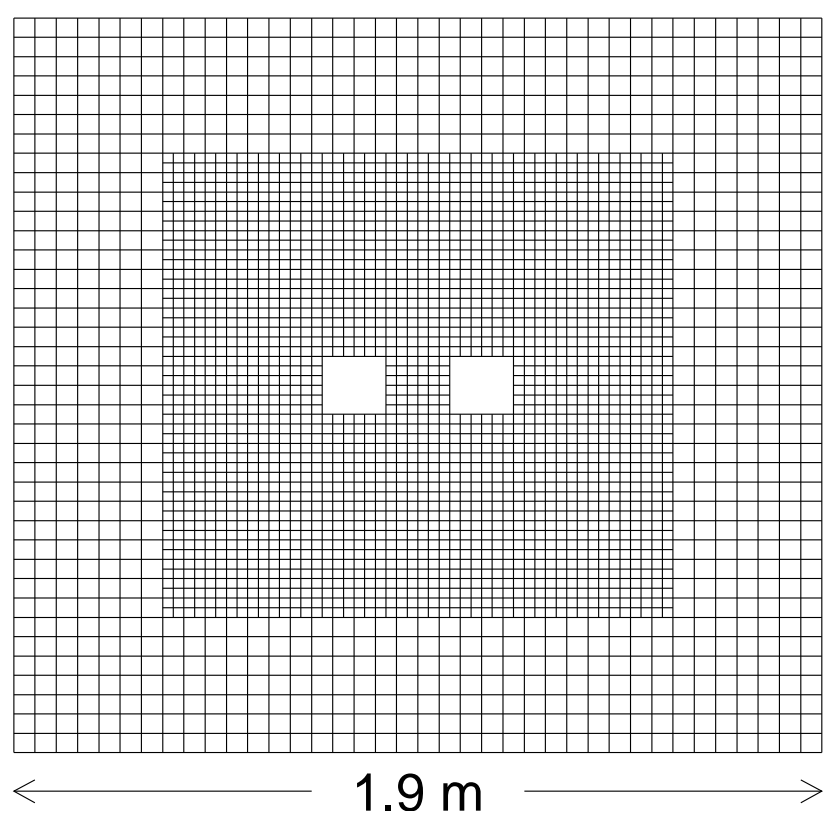

FIG. 5: Beamline view of the KTeV CsI calorimeter, showing the 868 larger outer crystals and the 2232 smaller inner crystals. Each beam hole size is $15 \times 15 \mathrm{~cm}^{2}$ and the two beam hole centers are separated by $0.3 \mathrm{~m}$.

obtain sub-percent energy resolution for electrons and photons.

The CsI scintillation light has two components: (i) a fast component with decay times of $10 \mathrm{~ns}$ and $36 \mathrm{~ns}$, and maximum light output at a wavelength of $315 \mathrm{~nm}$; (ii) a slow component with a decay time of $\sim 1 \mu \mathrm{s}$ and maximum light output at $480 \mathrm{~nm}$. To reduce accidental pile-up effects from the slow component, a Schott UV filter is placed between the crystal and the PMT. The filter reduces the total light output by $\sim 20 \%$, but increases the fast component fraction from about $80 \%$ to $90 \%$. The average light yield with the filter gives 20 photo-electrons per $\mathrm{MeV}$ of energy deposit. The CsI signal components discussed above include the effect of the PMT spectral response.

Digitizing electronics are placed directly behind the PMTs to minimize electronic noise $(<1 \mathrm{MeV})$. Each PMT is equipped with a custom 8-range digitizer to integrate the charge delivered by the PMT. This device, which is referred to as a "digital PMT" (DPMT) [24, has 16 bits of dynamic range with 8-bit resolution, and allows the measurement of energies from a few $\mathrm{MeV}$ to $100 \mathrm{GeV}$. In 1997, the digitization and readout operated at the Tevatron RF frequency of $53 \mathrm{MHz}$, and the PMT signal integration time was 114 ns (6 RF "buckets"), which permitted collection of approximately $96 \%$ of the fast scintillation component. In 1996, the readout frequency was $18 \mathrm{MHz}(\mathrm{RF} / 3)$ and the integration time was a factor of two longer than in 1997.

To convert measurements of integrated charge to en- ergy, a laser system is first used to calibrate the response from each DPMT. Then momentum-analyzed electrons from $K_{L} \rightarrow \pi^{ \pm} e^{\mp} \nu$ decays are used to calibrate the energy scale of each channel.

The laser system consists of a pulsed Nd:YAG laser, a fluorescent dye, and a variable filter to span the full dynamic range of the readout system. The laser light $(360 \mathrm{~nm})$ is distributed via 3100 quartz fibers to each individual crystal; the light level for each quadrant of the calorimeter is monitored with a PIN diode read out by a 20-bit ADC. Throughout the data taking, special hourlong laser scans to calibrate the readout system were performed roughly once per week during periods when beam was not available. Using these laser scan calibrations, deviations from a linear fit of the combined DPMT plus PMT response versus light level are less than $0.1 \%$ (rms) for each channel. During nominal data-taking, the laser operated at a fixed intensity with $1 \mathrm{~Hz}$ repetition rate to correct for short-term gain drifts that were typically $<0.2 \%$ per day.

To determine the energy scale in the calorimeter, we collected 600 million $K_{L} \rightarrow \pi^{ \pm} e^{\mp} \nu$ decays during the experiment; this number of events allows us to determine the energy scale for each channel with $\sim 0.03 \%$ precision every 1-2 days. The electron energy is determined by summing energies from a $3 \times 3$ "cluster" of large crystals or a $7 \times 7$ cluster of small crystals centered on the crystal with maximum energy. The cluster energy is corrected for shower leakage outside the cluster region, leakage at the beam-holes and calorimeter edges, and for channels with energies below the $\sim 4 \mathrm{MeV}$ readout threshold.

Figure 6a shows the $E / p$ distribution for electrons from $K_{e 3}$ decays, where $E / p$ is the ratio of cluster energy measured in the CsI calorimeter to momentum measured in the spectrometer. To avoid pion shower leakage into the electron shower, the $\pi^{ \pm}$is required to be at least $50 \mathrm{~cm}$ away from the $e^{\mp}$ at the CsI. The $E / p$ resolution has comparable contributions from both $E$ and $p$. The CsI energy resolution is obtained by subtracting the momentum resolution from the $E / p$ resolution, and is shown as a function of momentum in Fig. 6b. The energy resolution can be parameterized as $\sigma_{E} / E \simeq 2 \% / \sqrt{E} \oplus 0.4 \%$, where $E$ is in $\mathrm{GeV}$; the resolution is $1.3 \%$ at $3 \mathrm{GeV}$, the minimum cluster energy used in the $K \rightarrow \pi^{0} \pi^{0}$ analysis, and is better than $0.6 \%$ for energies above $20 \mathrm{GeV}$. The momentum dependence of the mean $E / p$ (Fig. Dec) shows that the average energy nonlinearity is $0.5 \%$ between 3 and $75 \mathrm{GeV}$. This energy nonlinearity is measured for each CsI channel and used as a correction.

Electromagnetic cluster positions are determined from the fraction of energy in neighboring columns and rows. The conversion from energy fraction to position is done using a map based on the uniform photon illumination across each crystal for $K \rightarrow \pi^{0} \pi^{0}$ data. The average position resolution for electrons is $1.2 \mathrm{~mm}$ for clusters in small crystals, and $2.4 \mathrm{~mm}$ for large crystals. 

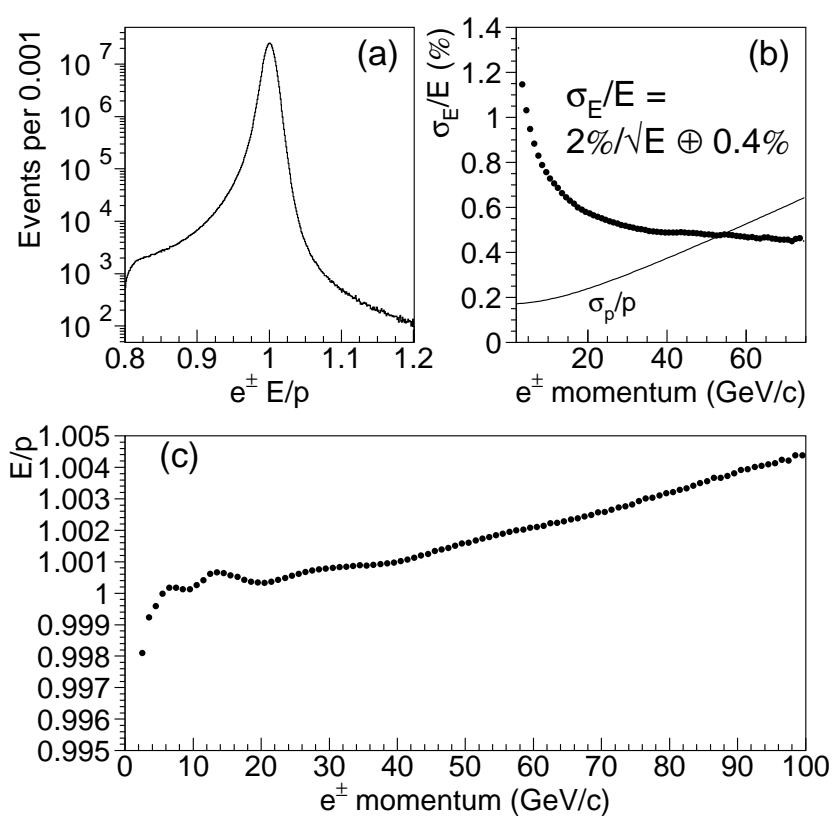

FIG. 6: (a) $E / p$ distribution based on electrons from $K_{L} \rightarrow \pi^{ \pm} e^{\mp} \nu$ decays, where $E$ is the energy measured in the CsI calorimeter and $p$ is the momentum measured in the spectrometer; (b) electron energy resolution vs. momentum (dots) obtained by subtracting the tracking resolution, shown by the smooth curve, from the $E / p$-resolution; (c) $E / p$-mean vs. momentum before the linearity correction is applied.

\section{The Regenerator}

$\mathrm{KTeV}$ uses an active regenerator as a source of $K_{S}$ decays. It consists mainly of $8410 \times 10 \times 2 \mathrm{~cm}^{3}$ scintillator modules (Fig. 7a). Each module is viewed by two photomultiplier tubes (PMTs), one from above and one from below. The downstream end of the regenerator (Fig. $7 \mathrm{~b}$ ) is a lead-scintillator sandwich, which is also viewed by two PMTs. This last module of the regenerator is used to define a sharp upstream edge for the kaon decay region in the regenerator beam.

At the average kaon momentum of $70 \mathrm{GeV} / c$, the magnitude of the regeneration amplitude is $|\rho| \sim 0.03$. The isoscalar carbon in the regenerator accounts for about $95 \%$ of the regeneration amplitude, which simplifies the model used to describe $\rho$ when extracting physics parameters (Sec. IVQ).

One can distinguish three main processes that contribute to $K_{S}$ regeneration. These are (i) "coherent" regeneration, which occurs in the forward direction, (ii) "diffractive" regeneration, in which target nuclei do not disintegrate but kaons scatter at finite angle, and (iii) "inelastic" regeneration, characterized by nuclear breakup and often by production of secondary particles. Only the decays of coherently regenerated kaons are used in the $\operatorname{Re}\left(\epsilon^{\prime} / \epsilon\right)$ analysis. The other processes are treated as background. (a)
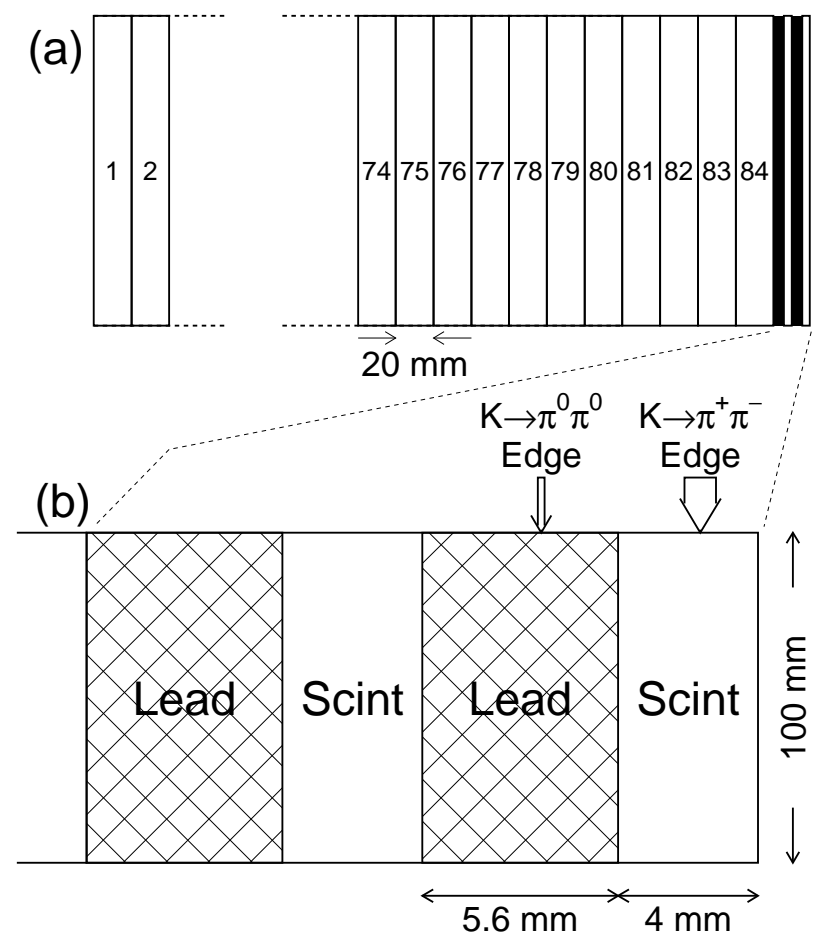

FIG. 7: (a) Diagram of the regenerator and (b) magnified view of the downstream end of the regenerator. PMTs are not shown. The dimension of each element is given in the figure. The kaon beam enters from the left. Vertical arrows above the lead and scintillator show the effective edge locations for $K \rightarrow \pi^{0} \pi^{0}$ and $K \rightarrow \pi^{+} \pi^{-}$decays; the width of each arrow indicates the uncertainty.

The $170 \mathrm{~cm}$ length of the regenerator corresponds to about two hadronic interaction lengths. This length maximizes coherent regeneration and suppresses diffractive regeneration [25]. The diffractive-to-coherent ratio is 0.09 for $K \rightarrow \pi \pi$ decays downstream of the regenerator, and is reduced by kinematic cuts in the analysis. The corresponding inelastic-to-coherent ratio is about 100. Since inelastic regenerator interactions typically leave energy deposits of a few $\mathrm{MeV}$ to $100 \mathrm{MeV}$ from the recoil nuclear fragments, this source of background is reduced using the regenerator PMT signals; events with more than $8 \mathrm{MeV}$ in any scintillator element are rejected. Inelastic interactions with production of secondary particles are suppressed further by other elements of the veto system. After all veto requirements, the level of inelastic scattering is reduced by a factor of a few thousand, making its contribution smaller than that of diffractive scattering.

The downstream edge of the regenerator defines the beginning of the regenerator-beam decay region for both the $K \rightarrow \pi^{+} \pi^{-}$and $K \rightarrow \pi^{0} \pi^{0}$ modes. A small fraction of decays inside the regenerator enters the signal sample because photons can pass through scintillator and lead, and because charged pions can exit the last regenerator module without depositing enough energy to be vetoed. In the fit to extract $\operatorname{Re}\left(\epsilon^{\prime} / \epsilon\right)$ (Sec. IVC), the median of 
the distribution of $K \rightarrow \pi \pi$ decays inside the regenerator is used to define a perfectly sharp "effective edge," $z_{\text {eff }}$. For the neutral decay mode, $z_{\text {eff }}$ is calculated using the known geometry and regeneration properties, and is $(-6.2 \pm 0.1) \mathrm{mm}$ from the downstream end of the regenerator; it is shown by the arrow above the lead in Fig. $7 \mathrm{~b}$. For the charged decay mode, $z_{\text {eff }}$ is determined by the veto threshold in the last regenerator module. The threshold is measured using muons collected with a separate trigger, and results in a $K \rightarrow \pi^{+} \pi^{-}$edge that is $(-1.65 \pm 0.45) \mathrm{mm}$ from the downstream edge of the regenerator; it is shown by the arrow above the scintillator in Fig. 7 $\mathrm{b}$. The uncertainty comes from the geometry of the PMT-scintillator assembly and the threshold measurement from muons.

\section{The Veto System}

The KTeV detector uses veto elements to reduce trigger rates, to reduce backgrounds, and to define sharp apertures and edges that limit the detector acceptance. The regenerator veto was discussed in the previous section.

Nine lead-scintillator $\left(16 X_{0}\right)$ photon veto counters are positioned along the beam-line, with five located upstream of the vacuum-window (Fig. 8a) and four located downstream of the vacuum-window. These nine veto counters detect escaping particles that would miss any of the drift chambers or the CsI calorimeter. Another $10 X_{0}$ photon veto is placed behind the CsI to detect photons that go through the beam-holes; this "beam-hole veto" mainly suppresses background from $K_{L} \rightarrow \pi^{0} \pi^{0} \pi^{0}$ decays. A scintillator bank behind $4 \mathrm{~m}$ of steel ( $z=192 \mathrm{~m}$ in Fig. 2 $)$ is used to veto muons, primarily from $K_{L} \rightarrow \pi^{ \pm} \mu^{\mp} \nu$ decays.

The upstream distribution of reconstructed kaon decays is determined mainly by the "Mask Anti" (MA, Fig $8 \mathrm{~b}$ ), which is a $16 X_{0}$ lead-scintillator sandwich located at $z=123 \mathrm{~m}$. The MA has two $9 \times 9 \mathrm{~cm}^{2}$ holes through which the neutral beams pass. At the downstream end of the detector, the CsI crystals around the beam-holes are partially covered by an $8.7 X_{0}$ tungstenscintillator "Collar Anti" (CA, Fig 9). In addition to defining a sharp edge, the $\mathrm{CA}$ veto rejects events in which more than $10 \%$ of a photon's energy is lost in a beam hole.

\section{Trigger and Data Acquisition}

$\mathrm{KTeV}$ uses a three-level trigger system to reduce the total rate of stored events to approximately $2 \mathrm{kHz}$, while efficiently collecting $K \rightarrow \pi \pi$ decays. The Level 1 trigger (L1) has no deadtime and makes a decision every 19 ns (corresponding to the beam RF-structure) using fast signals from the detector. The Level 2 trigger (L2) is based on more sophisticated processors and introduces a deadtime of $2-3 \mu \mathrm{s}$. When an event passes the Level 2 trigger, (a) Photon Veto

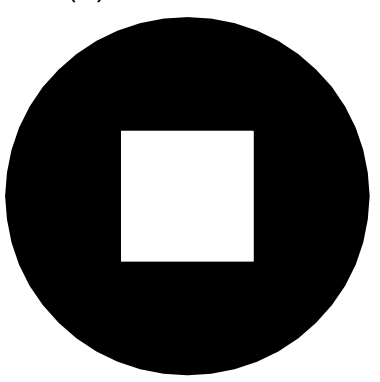

(b) Mask Anti (MA)

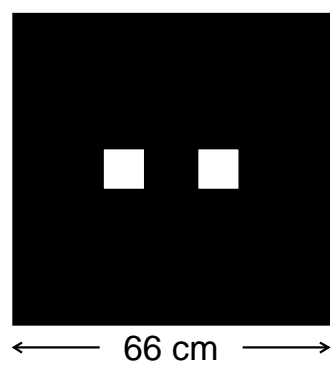

FIG. 8: (a) Transverse layout for one of five photon veto detectors between the regenerator and the vacuum-window (Fig. 1). The square aperture size varies, but is roughly 1 meter. (b) MA photon veto detector $(z=123 \mathrm{~m})$. The two MA beam hole sizes are $9 \times 9 \mathrm{~cm}^{2}$ and their centers are separated by $20 \mathrm{~cm}$. For both detectors the shaded region indicates the active veto area. The PMTs are at the outer edges of each detector.

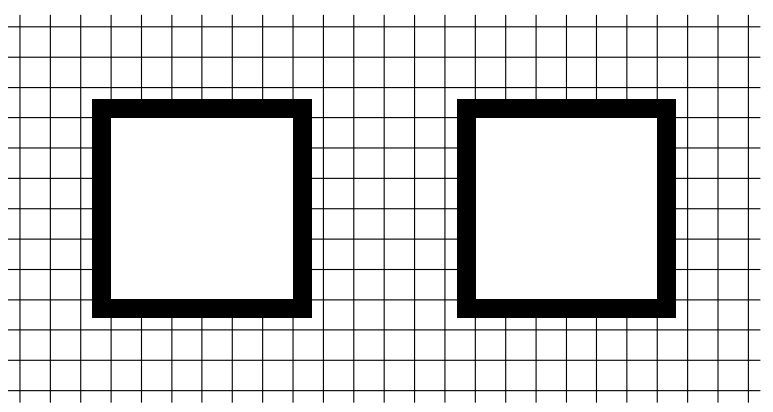

FIG. 9: The Collar Anti (CA) is shown as the two shaded "picture-frame" regions that cover the inner $60 \%(1.50 \mathrm{~cm})$ of the CsI crystals surrounding the beam holes. The neutral beams go into the page, and the two beam-hole centers are separated by $30 \mathrm{~cm}$. Wavelength shifting fibers transmit the scintillation light to PMTs at the edges of the calorimeter.

the entire detector is read out with an average deadtime of $15 \mu \mathrm{s}$. Each event is then sent to one of twenty-four 200-MHz SGI processors running a Level 3 (L3) software filter. An event passing Level 3 selection is written to a Digital Linear Tape for permanent storage. An independent set of ten $150-\mathrm{MHz}$ processors is used for online monitoring and calibration.

For rate reduction, the most important trigger element is the regenerator veto, which uses the signal from the downstream lead-scintillator sandwich plus signals from 3 of the 84 scintillator modules. This veto is applied in Level 1 triggers to reject events from the $2 \mathrm{MHz}$ of hadrons that interact in the regenerator. After applying the regenerator veto, there is still a $100 \mathrm{kHz}$ rate of kaon decays and another $100 \mathrm{kHz}$ rate of hadron interactions in the vacuum-window and drift chambers. Additional trigger requirements are used to reduce this $200 \mathrm{kHz}$ rate by about a factor of 100 to match the bandwidth of the data acquisition system. 
Separate triggers are defined for the signal $\pi^{+} \pi^{-}$and $\pi^{0} \pi^{0}$ modes. Each trigger is identical for the two beams and for both regenerator positions. The charged, neutral, and special purpose triggers are described below. Each section includes a brief summary of the trigger inefficiency, which is defined as the fraction of events that pass all analysis cuts, but fail the trigger. The inefficiencies are measured using decays collected in separate minimum-bias triggers.

Charged Decay Trigger: The Level 1 trigger for charged decays requires hits in the two drift chambers upstream of the magnet, and requires hits in the "trigger hodoscope" located $2 \mathrm{~m}$ upstream of the CsI. Each drift chamber is required to have at least one hit in both the $x$ and $y$ views. The trigger hodoscope consists of two $5 \mathrm{~mm}$ thick scintillation planes, each with 31 individually wrapped counters. There are small gaps between the counters, representing $1.1 \%$ of the area of each scintillation plane. The hodoscope counters are arranged to minimize the impact of these gaps; a particle traversing a gap in one plane cannot pass through a gap in the other plane. Each plane has two $14 \times 14 \mathrm{~cm}^{2}$ holes to allow the neutral beams to pass through without interacting. The trigger requires 1 or more hits in both planes, and 2 or more hits in at least one plane; this requirement allows for one of the charged particles to pass through a gap between the counters. The two hodoscope hits must also include both the upper and lower "regions," as well as the left and right regions. The defined hodoscope regions have sufficient overlap to prevent losses for $K \rightarrow \pi^{+} \pi^{-}$events, except for decays in which a pion passes through a scintillator gap in the central region defined by $|y|<7 \mathrm{~cm}$. To reduce the trigger rate from non- $\pi^{+} \pi^{-}$kaon decays, signals from the veto system are used to reject events at the first trigger level. The muon veto is used to suppress $K_{L} \rightarrow \pi^{ \pm} \mu^{\mp} \nu$ decays, and the photon vetos downstream of the vacuum-window are used to reject decays with a photon in the final state.

The Level 2 trigger requires that the drift chamber hits in the $y$-view be consistent with two tracks from a common vertex; to reduce signal loss from inefficient wires, a missing hit is allowed. The L3 filter selects $K \rightarrow \pi^{+} \pi^{-}$ candidates by reconstructing two charged tracks in the spectrometer; to reject $K_{L} \rightarrow \pi^{ \pm} e^{\mp} \nu$ and $K_{L} \rightarrow \pi^{+} \pi^{-} \pi^{0}$ decays, the $\pi^{+} \pi^{-}$mass is required to be greater than $450 \mathrm{MeV} / c^{2}$ and $E / p$ is required to be less than 0.9 for both tracks. For CsI calibration and detector acceptance studies, $1 / 7$ of the $K_{e 3}$ candidates are kept by requiring $E / p>0.9$ for one of the tracks.

The total charged trigger inefficiency is $0.5 \%$, and is mainly from the $0.3 \%$ loss due to gaps between the scintillator counters. The drift chamber requirements result in a $0.1 \%$ inefficiency. Accidental effects result in a $0.06 \%$ loss, and there is a $0.04 \%$ loss from the trigger hardware. The Level 3 inefficiency is $0.09 \%$.

Neutral Decay Trigger: The Level 1 trigger for neutral decays is based on the total energy deposited in the
CsI calorimeter. Using a 3100 channel analog sum, the threshold is $24 \mathrm{GeV}$ and the $10-90 \%$ turn-on width is $7 \mathrm{GeV}$. The photon vetos downstream of the vacuumwindow are used in the Level 1 trigger to reduce the rate from $K_{L} \rightarrow \pi^{0} \pi^{0} \pi^{0}$ decays. The Level 2 trigger uses a Hardware Cluster Counter [27] to count clusters of energy above $1 \mathrm{GeV}$ in the CsI. Four clusters are required for $K \rightarrow \pi^{0} \pi^{0}$ decays, and a separate six-cluster trigger is pre-scaled by five to collect $K_{L} \rightarrow \pi^{0} \pi^{0} \pi^{0}$ decays. The Level 3 filter requires that the invariant mass be greater than $450 \mathrm{MeV} / c^{2}$ for both the $\pi^{0} \pi^{0}$ and $\pi^{0} \pi^{0} \pi^{0}$ final states.

The neutral energy-sum trigger inefficiency has two components: a $0.6 \%$ inefficiency from early accidental effects and an inefficiency of $4 \times 10^{-5}$ from the trigger hardware. The Level 2 trigger inefficiency of $0.4 \%$ comes from the Hardware Cluster Counter. The Level 3 inefficiency is $0.01 \%$.

Other Triggers: In addition to the triggers used to select specific decay modes, several special-purpose triggers are used for monitoring. These include (i) a "pedestal" trigger, which reads out the entire CsI calorimeter a few times per $20 \mathrm{~s}$ spill, (ii) a laser trigger to monitor the CsI (Sec. II C 2), (iii) a muon trigger that requires a muon hodoscope signal instead of using it in veto, (iv) a charged mode trigger that does not use the regenerator veto, (v) a trigger using only the Level 1 CsI analog sum with no Level 2 requirement, and (vi) an "accidental" trigger to record random activity in the detector that is proportional to the instantaneous intensity of the proton beam; triggers iii-vi are heavily pre-scaled. For the accidental trigger, we use a telescope consisting of three scintillation counters, each viewed by a photomultiplier tube. It is located $1.8 \mathrm{~m}$ from the $\mathrm{BeO}$ target and is oriented at an angle of $90^{\circ}$ with respect to the beam axis. The target is viewed by the counters through a $6.4 \times 6.4 \mathrm{~mm}^{2}$ hole in the stainless steel shielding around the target. A coincidence of signals in all three counters generates an accidental trigger.

Trigger Rates: Under nominal conditions, the total rates passing $\mathrm{L} 1, \mathrm{~L} 2$, and $\mathrm{L} 3$ are 40,10 , and $2 \mathrm{kHz}$, respectively; this L3 rate corresponds to approximately 40, 000 events written to tape each minute. The deadtime, which is common to all triggers, is about $33 \%$ with roughly equal contributions from Level 2 and readout.

\section{Data Collection}

The data used in this analysis were collected in two distinct "E832" periods: October-December in 1996 and April-July in 1997. In these periods, there were about 200 billion kaon decays between the defining collimator and the CsI calorimeter, of which 5 billion events were written to $300015-\mathrm{Gb}$ tapes. About $5 \%$ of the events are used to select the $K \rightarrow \pi \pi$ sample, and the remaining 
$95 \%$ of the events are used to understand the detector. The neutral mode data from 1996 and 1997 are used. For the charged mode, the 1996 sample is not used because the Level 3 rejection of delayed hits in the drift chambers led to a $20 \%$ signal loss that is difficult to simulate. In 1997, the Level 3 tracking was modified to avoid this loss. Excluding the 1996 charged mode data has a negligible effect on the overall statistical and systematic uncertainty in $\operatorname{Re}\left(\epsilon^{\prime} / \epsilon\right)$.

To ensure that the final data sample is of high quality, periods in which there are known detector problems are excluded. The CsI calorimeter readout suffered DPMT failures at a rate of about one per day, and was the most significant detector problem. Each DPMT failure was identified immediately by the online monitoring, and then repaired. Calibrations were frequent enough so that every repaired DPMT can be calibrated offline. The final data sample does not include periods in which there were dead CsI channels or dead cells in the drift chambers. Approximately $12 \%$ of the data on tape are rejected because of detector problems.

\section{E. Monte Carlo Simulation}

The Monte Carlo (MC) simulation consists of three main steps. The first step is kaon generation at the $\mathrm{BeO}$ target and propagation along the beamline to the decay point. The second step is kaon decay into an appropriate final state, and tracing of the decay products through the detector. The last step is to simulate the detector response including digitization of the detector signals. The simulated event format and analysis are the same as for the data.

The detector geometry used in the simulation comes from survey measurements and data. The survey measurements are used for the transverse dimensions and $z$ locations of beamline and detector elements. The transverse offsets and rotations of the drift chambers, relative to the CsI calorimeter, are determined using various data samples as discussed in Section II C 1. The Mask-Anti and Collar-Anti (Sec. II C 4) aperture sizes and locations are determined using electrons from $K_{L} \rightarrow \pi^{ \pm} e^{\mp} \nu$ decays.

As a result of the high flux of kaons and neutrons in the $\mathrm{KTeV}$ apparatus, there can be underlying accidental activity in the detector that is unrelated to the kaon decay. After applying veto cuts, the average accidental energy under each CsI cluster is a few $\mathrm{MeV}$, and there are roughly 20 extra in-time drift chamber hits. To simulate these effects, we use data events from the accidental trigger (Sec. II C 5) to add the underlying accidental activity to each generated MC event. In the CsI calorimeter and veto system, activity from an accidental event is added to the MC energy deposits in a straightforward manner. The procedure for including accidental activity in the drift chamber simulation, however, is more complicated because an empirical model is needed to describe how an accidental hit can obscure a signal hit that arrives later on the same wire.

\section{Kaon Propagation and Decay}

The kaon energy spectrum and the relative flux of $K^{0}$ and $\overline{K^{0}}$ states produced at the target are based on a parameterization [28] that is tuned to match $\mathrm{KTeV}$ $K \rightarrow \pi^{+} \pi^{-}$data. The $z$ position of each kaon decay is chosen based on the calculated $z$ distribution for the initial $K^{0}$ or $\overline{K^{0}}$ state, and accounts for interference between $K_{L}$ and $K_{S}$.

The simulation propagates the $K^{0}$ and $\overline{K^{0}}$ amplitudes along the beamline, accounting for regeneration and scattering in the absorbers and the regenerator. Some smallangle kaon scatters in the absorbers $(z \sim 19 \mathrm{~m}$, Fig. 11) can pass through the collimator system and satisfy the $K \rightarrow \pi \pi$ analysis requirements. The upstream collimators are modeled as perfectly absorbing, while scattering in the defining collimators and the regenerator are treated using models that are tuned to data (Sections III F 2. III F 3).

For $K \rightarrow \pi^{+} \pi^{-}$decays, radiative corrections due to inner Bremsstrahlung are included [29]. We do not simulate the direct emission part of the radiative spectrum since the invariant mass cut used in the analysis essentially eliminates this component. For the $K \rightarrow \pi^{0} \pi^{0}$ mode, only the four photon final state is considered.

GEANT [30 is used to parameterize scattering of final state charged particles in the vacuum-window, helium bags, drift chambers, trigger hodoscope, and the steel. Electrons can undergo Bremsstrahlung, photons can convert to $e^{+} e^{-}$pairs, and charged pions can decay into a muon and a neutrino; these secondary particles are traced through the detector.

\section{Simulation of the Drift Chambers}

The Monte Carlo traces each charged particle through the drift chambers, and the hit position at each drift chamber plane is converted into a TDC value. The position resolutions measured in data are used to smear the hit positions, and the inverse of the $x(t)$ map is used to convert the smeared hit position into a drift time.

The simulation includes four effects that cause drift chamber signals to be corrupted or lost.

1. "Wire inefficiency" results in no in-time TDC hit. The inefficiency is measured in $1 \mathrm{~cm}$ steps along each wire of each chamber. The average single-hit inefficiency is less than $1 \%$. Since the inefficiency increases with distance from the wire, the measured inefficiency profile within the cell is used in the simulation.

2. A "delayed hit" results in a hit-pair with a sumof-distance (SOD) that is more than $1 \mathrm{~mm}$ greater 
than the nominal cell size, and therefore does not satisfy the hit-pair requirement (Sec. II C 1). The delayed hit probability is a few percent in the regions where the neutral beams pass through the drift chambers, and about $1 \%$ over the rest of the chamber area. The effect is modeled by distributing primary drift electrons along the track using a Poisson distribution with an average interval of $340 \mu \mathrm{m}$, and then generating a composite signal at the sense wire. A delayed hit occurs when the signal from the nearest ionization cluster is below threshold, but the composite pulse from all ionization clusters is above threshold. The MC threshold is determined empirically by matching the MC delayed hit probability to data; the delayed hit probability in data is measured in $1 \mathrm{~cm}$ steps along each wire.

3. When an "in-time accidental" hit arrives before a signal hit on the same wire, the accidental hit is used instead of the signal hit because the tracking program considers only the first in-time hit. For roughly $0.7 \%$ of the hit-pairs, this effect causes a "low-SOD" that is more than $1 \mathrm{~mm}$ below the nominal cell size, and therefore does not pass the hitpair requirement. An "early accidental" hit prior to the in-time window can also obscure a signal hit on the same wire for two reasons. First, the discriminator has a deadtime of 42 ns during which the wire is $100 \%$ inefficient. Second, large analog pulses can stay above the discriminator threshold longer than $42 \mathrm{~ns}$; the variation of the pulse-length is modeled and tuned to data.

4. Delta rays also cause a low-SOD for $0.5 \%$ of the hitpairs. In the Monte Carlo, delta rays are generated in the same cell as the track, and the rate is tuned to match the low-SOD distribution in data.

The quality of the drift chamber simulation is illustrated by the SOD distribution in Fig. 4. Both the low and high-side tails in the data are well simulated.

\section{Simulation of the CsI Calorimeter}

The Monte Carlo simulation is used to predict the energy deposit in each crystal when kaon decay products hit the calorimeter. In particular, the MC is needed to model energy leakage in the beam-holes and at the outer edges of the calorimeter, and to model nearby showers that share energy. A library of GEANT-based 30 electron and photon showers is used to simulate electromagnetic showers in the calorimeter. In the shower generation, each electron or photon is incident on the central crystal of a $13 \times 13$ array of small crystals $\left(32.5 \times 32.5 \mathrm{~cm}^{2}\right)$. The showers are generated in 6 energy bins from 2 to 64 $\mathrm{GeV}$, and in $x, y$ position bins. The position bin spacing varies from $7 \mathrm{~mm}$ at the crystal center to $2 \mathrm{~mm}$ at the edge; this binning matches the variation in reconstructed position resolution, which is better for particles incident near the edge of a crystal. Outside the $13 \times 13$ array, a GEANT-based parameterization is used instead of a library of individual showers; this parameterization models energy deposits in a $27 \times 27$ array. Energy leakage across the beam-holes is modeled based on electron data from $K_{L} \rightarrow \pi^{ \pm} e^{\mp} \nu$ decays.

To simulate the DPMT response, the energy deposit in each crystal is distributed among six consecutive RF buckets according to the measured time profile of the scintillation light output. In each 19 ns wide RF bucket, the energy is smeared to account for photo-statistics, and random activity from an accidental trigger is added. Each channel is digitized using the inverse of the calibrations obtained from data. A channel is processed if the digitized signal exceeds the $4 \mathrm{MeV}$ readout threshold that was applied during data-taking.

In addition to simulating electromagnetic showers, we also simulate the calorimeter response to charged pions and muons. The energy deposits from charged pions are based on a library of GEANT-based showers using a $50 \times 50$ array of small crystals. A continuous energy distribution is generated in $x, y$ position bins with $4 \mathrm{~mm}$ separation. For muons, the average CsI energy deposit is $320 \mathrm{MeV}$, and is simulated using the Bethe-Bloch energy loss formula.

\section{Simulation of the Trigger}

The $\mathrm{KTeV}$ Monte Carlo includes a simulation of the Level 1 and Level 2 triggers, and the Level 3 software filter. For the $K \rightarrow \pi^{+} \pi^{-}$trigger, the most important effect to simulate is the $0.3 \%$ inefficiency due to scintillator gaps in the hodoscope just upstream of the CsI calorimeter. The gap sizes and positions are measured in data using the $K_{e 3}$ sample. The simulation also includes the drift chamber signals at both Level 1 and Level 2. For the $K \rightarrow \pi^{0} \pi^{0}$ trigger, we use $K_{L} \rightarrow \pi^{ \pm} e^{\mp} \nu$ decays to determine the calorimeter energy-sum threshold and turn-on width, and to measure the Hardware Cluster Counter threshold for each CsI channel.

\section{DATA ANALYSIS}

The analysis is designed to identify $K \rightarrow \pi \pi$ decays while removing poorly reconstructed events that are difficult to simulate, and to reject background. The following sections describe the analysis and the associated systematic uncertainties in $\operatorname{Re}\left(\epsilon^{\prime} / \epsilon\right)$. When discussing systematic uncertainties, we typically estimate a potential shift $s \pm \sigma_{s}$, where $s$ is the shift in $\operatorname{Re}\left(\epsilon^{\prime} / \epsilon\right)$ and $\sigma_{s}$ is the accompanying statistical uncertainty. We convert the shift to a symmetric systematic error, $\Delta_{s}$, such that the range $\left[-\Delta_{s},+\Delta_{s}\right]$ includes $68.3 \%$ of the area of a 
Gaussian with mean $s$ and width $\sigma_{s}$ :

$$
\frac{1}{\sigma_{s} \sqrt{2 \pi}} \int_{-\Delta_{s}}^{+\Delta_{s}} d x \exp \left[-\frac{(x-s)^{2}}{2 \sigma_{s}^{2}}\right]=0.683 .
$$

Note that $\Delta_{s}=\sigma_{s}$ when $s=0$; when $s>\sigma_{s}, \Delta_{s} \approx$ $s+\sigma_{s} / 2$.

\section{A. Common Features of $\pi^{+} \pi^{-}$and $\pi^{0} \pi^{0}$ Analyses}

Although many details of the charged and neutral decay mode analyses are different, several features are common to reduce systematic uncertainties. For each decay mode, the same cuts are applied to decays in the vacuum and regenerator beams, so that most systematic uncertainties cancel in the single ratios used to measure $\left|\eta_{+-} / \rho\right|^{2}$ and $\left|\eta_{00} / \rho\right|^{2}$.

Since the regeneration amplitude $\rho$ depends on the kaon momentum, we select an identical 40-160 GeV/c kaon momentum range for both the charged and neutral decay modes. The $40 \mathrm{GeV} / c$ cut is chosen because of the rapidly falling detector acceptance at lower kaon momenta; the higher momentum cut is a compromise between slightly higher statistics and target- $K_{S}$ contamination. We also use the same $z$-vertex range of $110-158 \mathrm{~m}$ for each decay mode. The $110 \mathrm{~m}$ cut is chosen to be well upstream of the Mask Anti and therefore removes very few decays in the vacuum beam; this cut removes no events in the regenerator beam. The downstream $z$ vertex requirement avoids background from beam interactions in the vacuum-window.

To simplify the treatment of background from kaons that scatter in the regenerator, the veto requirements for the charged and neutral mode analyses are as similar as possible. In both analyses, the main reduction in background from regenerator scattering comes from the requirement that there be less than $8 \mathrm{MeV}$ in every regenerator module (Sec. II C 3). Interactions in the regenerator that are close in time to a $K \rightarrow \pi \pi$ decay can add significant activity in the detector, resulting in events that are difficult to reconstruct. To avoid this problem, the trigger signal from the regenerator is used to reject events in a $57 \mathrm{~ns}$ wide window ( $3 \mathrm{RF}$ buckets), which removes events immediately following or just prior to an interaction in the regenerator. In addition to the regenerator veto, we require that there be less than 150 (300) $\mathrm{MeV}$ in the photon vetos upstream (downstream) of the vacuum-window, and less than $300 \mathrm{MeV}$ in the Mask Anti; note that the photon veto thresholds refer to equivalent photon energy.

\section{B. $K \rightarrow \pi^{+} \pi^{-}$Reconstruction and Selection}

The strategy to identify $K \rightarrow \pi^{+} \pi^{-}$decays is to reconstruct two well-measured tracks in the spectrometer, and to reduce backgrounds with particle identification and kinematic requirements.
The spectrometer reconstruction begins by finding $y$ tracks using all four drift chambers. In the $x$-view, separate segments are found upstream of the magnet using DC1 and DC2, and downstream of the magnet using DC3 and DC4. The extrapolated upstream and downstream $x$-track segments typically match to within $0.5 \mathrm{~mm}$ at the center of the magnet. To reduce sensitivity to multiplescattering and magnetic fringe fields between the drift chambers, only a loose match of $6 \mathrm{~mm}$ is required at the magnet. If two $x$-tracks and two $y$-tracks are found, the reconstruction continues by extrapolating both sets of tracks upstream to define an $x-z$ and $y-z$ vertex. The difference between these two vertices, $\Delta z_{v t x}$, is used to define a vertex- $\chi^{2}$,

$$
\chi_{v t x}^{2} \equiv\left(\Delta z_{v t x} / \sigma_{\Delta z}\right)^{2},
$$

where $\sigma_{\Delta z}$ is the resolution of $\Delta z_{v t x}$. This resolution depends on momentum and opening angle, and accounts for multiple scattering effects. The two $x$-tracks and two $y$-tracks are assumed to originate from a common vertex if $\chi_{v t x}^{2}<100$; this $\chi_{v t x}^{2}$ requirement is sufficiently loose to remain insensitive to the tails in the multiple scattering distribution. At this stage, the $x$ and $y$ tracks are independent. To determine the full particle trajectory, the $x$ and $y$ tracks are matched to each other based on their projections to CsI clusters; the track projections to clusters must match within $7 \mathrm{~cm}$.

After combining the $x$ and $y$ tracks, the reconstructed $z$-vertex resolution is about $30 \mathrm{~cm}$ near the regenerator and $5 \mathrm{~cm}$ near the vacuum-window. Each particle momentum is determined from the track bend-angle in the magnet and a precise B-field map.

An ideal event with two charged tracks results in 32 hits in the four chambers. About $40 \%$ of the events have at least one missing hit or a hit-pair that does not reconstruct a proper sum-of-distance (SOD) value. The tracking program can reconstruct events with many of these defects along the tracks. The overall single-track reconstruction inefficiency is measured to be $1 \%$ using $K_{L} \rightarrow \pi^{+} \pi^{-} \pi^{0}$ decays.

An event is assigned to the regenerator beam if the regenerator $x$-position has the same sign as the $x$ coordinate of the kaon trajectory at the downstream face of the regenerator; the event is assigned to the vacuum beam if the signs are different. For $K_{L} \rightarrow \pi^{ \pm} e^{\mp} \nu$ decays, this beam assignment definition cannot be used because of the missing neutrino; instead we compare the $x$-coordinate of the decay vertex to that of the regenerator.

The reconstruction described above is used for kaon decays with two charged tracks in the final state. The following selection criteria are specific to the $K \rightarrow \pi^{+} \pi^{-}$ channel, and are designed mainly to reduce background from semileptonic decays and kaon scattering.

Requiring $E / p<0.85$ for each track reduces the $K_{L} \rightarrow \pi^{ \pm} e^{\mp} \nu$ background by a factor of 1000 . The $K_{L} \rightarrow \pi^{ \pm} \mu^{\mp} \nu$ background is rejected largely by the muon veto in the Level 1 trigger. Since low momentum 
muons may range out in the $4 \mathrm{~m}$ of steel before depositing energy in the muon veto, we also require each track to have a momentum $p>8 \mathrm{GeV} / c$. To reject radiative $K \rightarrow \pi^{+} \pi^{-} \gamma$ decays, events with an isolated electromagnetic cluster above $1.0 \mathrm{GeV}$ are removed if the cluster is at least $20 \mathrm{~cm}$ away from both extrapolated pion track positions at the CsI; the pion-photon separation requirement avoids removing events that have satellite clusters from hadronic interactions. To remove background from $\Lambda \rightarrow p \pi^{-}$and $\bar{\Lambda} \rightarrow \bar{p} \pi^{+}$decays, the higher momentum track is assumed to be from a proton (or antiproton) and the event is rejected if the proton-pion invariant mass is within $3.5 \mathrm{MeV}$ of the known $\Lambda$ mass.

To provide additional background rejection of semileptonic and $K \rightarrow \pi^{+} \pi^{-} \gamma$ decays, the $\pi^{+} \pi^{-}$invariant mass is required to be in the range $488-508 \mathrm{MeV} / \mathrm{c}^{2}$. Figures $10 \mathrm{a}, \mathrm{b}$ show the invariant mass distributions for the vacuum and regenerator beams after all other selection cuts. The shapes of the vacuum and regenerator beam distributions are nearly the same, and have an RMS resolution of $\sim 1.6 \mathrm{MeV} / \mathrm{c}^{2}$. The low-mass tail is mainly due to the presence of $K \rightarrow \pi^{+} \pi^{-} \gamma$ events in both beams. The tails in the vacuum beam distribution also include background from semileptonic decays.
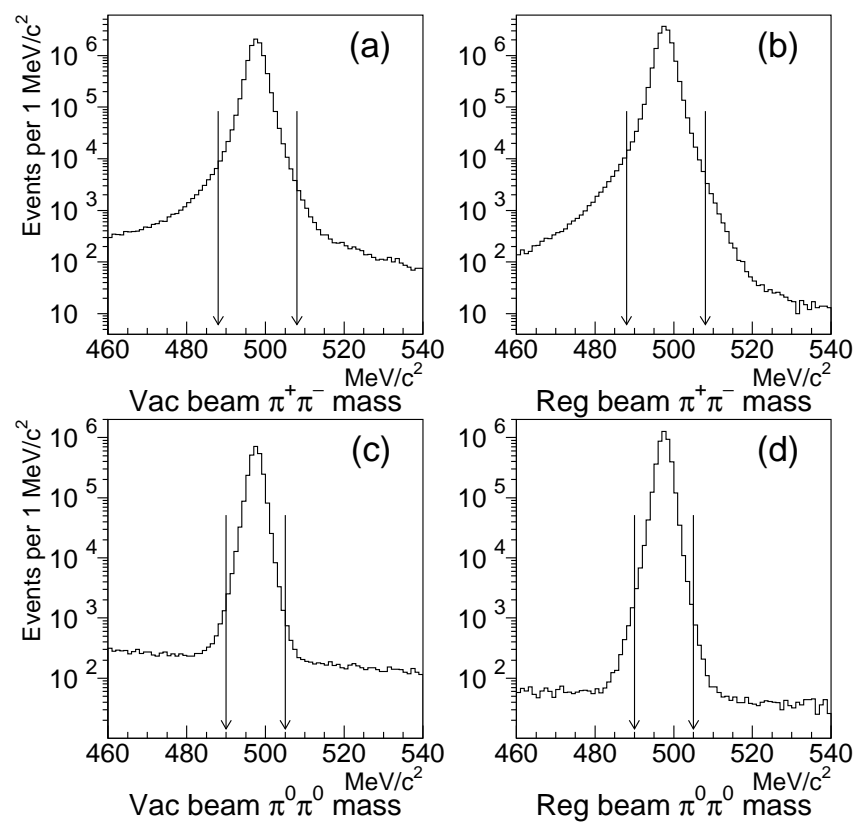

FIG. 10: Invariant mass distributions for (a) vacuum-beam $\pi^{+} \pi^{-}$, (b) regenerator-beam $\pi^{+} \pi^{-}$, (c) vacuum-beam $\pi^{0} \pi^{0}$, (d) regenerator-beam $\pi^{0} \pi^{0}$. All other analysis cuts are applied. The vertical arrows indicate the accepted mass range.

Background from kaon scattering in the regenerator is suppressed mainly by the regenerator veto requirement. To reduce this background further, a cut is made on the $\pi^{+} \pi^{-}$transverse momentum, which also rejects events from kaons that scatter in the defining collimator. As illustrated in Fig. 11, the total momentum of the $\pi^{+} \pi^{-}$ system $(\vec{p})$ is projected back to a point $\left(\mathrm{R}_{\text {reg }}\right)$ in the plane containing the downstream face of the regenerator, and $\vec{p}_{T}$ is the momentum component that is transverse to the line connecting $\mathrm{R}_{\text {reg }}$ to the target. This definition of $\vec{p}_{T}$ is used for both beams, and is optimized to distinguish between scattered and unscattered kaons in the regenerator beam. The data and $\mathrm{MC} p_{T}^{2}$ distributions in both beams are shown in Fig. 12, the selection cut, $p_{T}^{2}<250 \mathrm{MeV}^{2} / c^{2}$, is shown by the arrows.

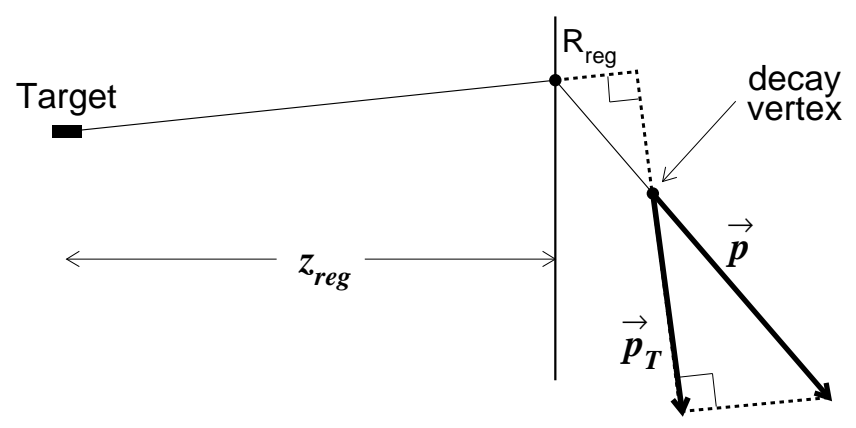

FIG. 11: Illustration of the transverse momentum, $\vec{p}_{T}$, for a kaon that scatters in the regenerator and then decays at the point labeled "decay vertex." The line connecting the target to $\mathrm{R}_{\text {reg }}$ represents the kaon trajectory upstream of the regenerator, $\vec{p}$ is the measured momentum of the $\pi^{+} \pi^{-}$system, and $z_{\text {reg }}$ is the distance from the target to the downstream end of the regenerator.
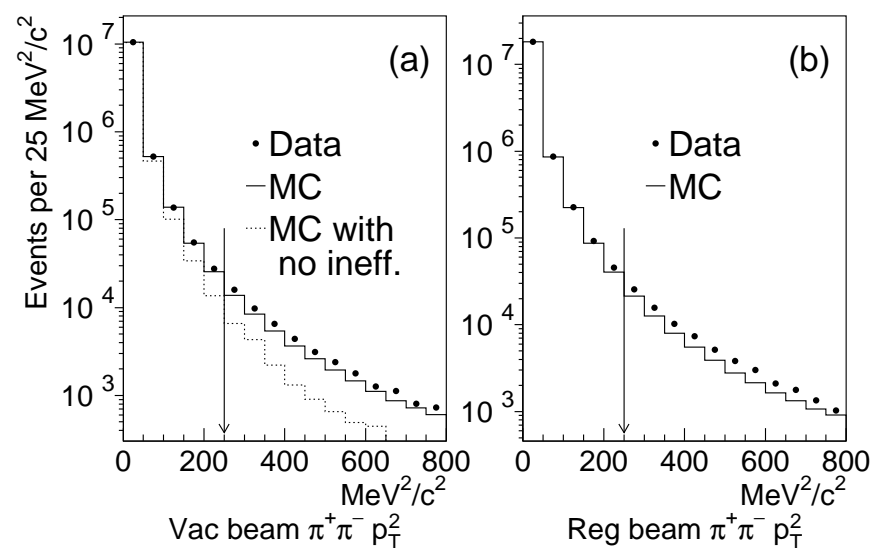

FIG. 12: $\pi^{+} \pi^{-} p_{T}^{2}$ distributions after all other $K \rightarrow \pi^{+} \pi^{-}$ analysis cuts in (a) the vacuum beam and (b) the regenerator beam. The background-subtracted data are shown as dots, and the histogram shows the MC prediction. The selection cut is shown by the arrows. The dotted histogram in the vacuum beam shows the MC prediction without the effects of wire inefficiency, delayed hits, and accidentals (Sec. II E 2).

Next, we describe selection requirements that define apertures. For $K \rightarrow \pi^{+} \pi^{-}$, unlike $K \rightarrow \pi^{0} \pi^{0}$ decays, the edges of the Mask Anti and Collar Anti vetos are not useful apertures because of the difficulty in simulating the interactions of charged pions. For the MA, a charged pion can pass through a small amount of lead-scintillator near 
TABLE I: Systematic uncertainties in $\operatorname{Re}\left(\epsilon^{\prime} / \epsilon\right)$ from the charged mode trigger and analysis. The uncertainties in the 3rd column, which also appear in the systematics summary (Table VIII), are the quadratic sum of contributions in the 2nd column.

\begin{tabular}{|c|c|c|}
\hline $\begin{array}{l}\text { Source of } \\
\text { uncertainty }\end{array}$ & \multicolumn{2}{|c|}{$\begin{array}{c}\text { Uncertainty on } \operatorname{Re}\left(\epsilon^{\prime} / \epsilon\right) \\
\left(\times 10^{-4}\right)\end{array}$} \\
\hline Trigger & & 0.58 \\
\hline L1 \& L2 & 0.20 & \\
\hline L3 filter & 0.54 & \\
\hline Track reconstruction & & 0.32 \\
\hline Alignment and calibration & 0.28 & \\
\hline Momentum scale & 0.16 & \\
\hline Selection efficiency & & 0.47 \\
\hline$p_{T}^{2}$ cut & 0.25 & \\
\hline DC efficiency modeling & 0.37 & \\
\hline DC resolution modeling & 0.15 & \\
\hline Apertures & & 0.30 \\
\hline Wire spacing & 0.22 & \\
\hline Regenerator edge & 0.20 & \\
\hline
\end{tabular}

the edge without leaving a veto signal, and multiple scattering in the MA results in a poorly reconstructed vertex. For the CA, the difficulty is with pions that miss the CA, but interact in the CsI calorimeter; backscattering from these pion interactions can still deposit sufficient energy in the CA to produce a veto signal. To avoid these apertures, we require that the track projections be away from the physical boundaries of the veto detectors. In a similar fashion, the outer CsI aperture is defined by track projections so that the acceptance does not depend on the CsI energy profile from charged pions.

If two tracks pass within the same drift chamber cell or adjacent cells, a hit from one track can obscure a hit from the other track. Since this effect is difficult to simulate, we use a track-separation requirement of two offset-cells (Fig. 3) between the tracks in both the $x$ and $y$ views for each chamber; offset-cells are used so that tracks near a wire, which have the poorest position resolution, are not near the boundary which defines the track-separation cut. This track-separation requirement results in an effective inner aperture.

\section{Systematic Uncertainties from $\pi^{+} \pi^{-}$Trigger, Reconstruction, and Selection}

In this section, we discuss the $\operatorname{Re}\left(\epsilon^{\prime} / \epsilon\right)$ systematic uncertainties from the charged mode trigger, reconstruction, and selection, which are summarized in Table If. Systematic uncertainties related to background and acceptance are discussed in Sections III F 5 and IV A.

Trigger: The Level 1 and Level 2 trigger requirements are studied with $K_{e 3}$ events collected in a trigger based only on CsI calorimeter energy, and contribute a $0.20 \times 10^{-4}$ uncertainty to $\operatorname{Re}\left(\epsilon^{\prime} / \epsilon\right)$. The L3 uncer- tainty is determined using a 1\% sample of charged triggers that pass L1 and L2, and are saved without requiring L3. From this sample, $3 \times 10^{5}$ events pass all $K \rightarrow \pi^{+} \pi^{-}$ analysis cuts. Applying the L3 requirement to this sample results in a $2.2 \sigma$ shift in the vacuum-to-regenerator ratio; the same analysis on $\mathrm{MC}$ events results in no shift from L3. These shifts in the vacuum-to-regenerator ratio correspond to a $(0.45 \pm 0.20) \times 10^{-4}$ shift in $\operatorname{Re}\left(\epsilon^{\prime} / \epsilon\right)$, which leads to an uncertainty of $0.54 \times 10^{-4}$ using Eq. 局.

Trajectory and Momentum Reconstruction: The effects of detector misalignment (Sec. II C 1 ) are studied by evaluating the change in $\operatorname{Re}\left(\epsilon^{\prime} / \epsilon\right)$ as the following are changed within their measured uncertainties: transverse chamber offsets and rotations, non-orthogonality between the $x$ and $y$ wire planes, and the $z$-locations of the drift chambers. The time-to-distance calibration is varied to change the average SOD value within its uncertainty. A $R e\left(\epsilon^{\prime} / \epsilon\right)$ uncertainty of $0.28 \times 10^{-4}$ is assigned based on these tests. The kaon mass is known to $0.031 \mathrm{MeV}$ [31], leading to a momentum-scale uncertainty of $1 \times 10^{-4}$, and a $\operatorname{Re}\left(\epsilon^{\prime} / \epsilon\right)$ uncertainty of $0.16 \times 10^{-4}$.

Selection Efficiency: In the charged mode analysis, $p_{T}^{2}$ is the only variable for which $\operatorname{Re}\left(\epsilon^{\prime} / \epsilon\right)$ is sensitive to the cut value. Figure 12 shows the $p_{T}^{2}$ data-MC comparison in both beams after background subtraction (Sec. III F), and also illustrates the importance of simulating details of the drift chamber performance. Increasing the $p_{T}^{2}$ cut value from 250 to $500 \mathrm{MeV}^{2} / c^{2}$ changes $\operatorname{Re}\left(\epsilon^{\prime} / \epsilon\right)$ by $(-0.23 \pm 0.05) \times 10^{-4}$, leading to a systematic uncertainty of $0.25 \times 10^{-4}$. There is no further statistically significant variation if the $p_{T}^{2}$ cut value is increased beyond $500 \mathrm{MeV}^{2} / c^{2}$.

The uncertainty in modeling the drift chamber efficiency is related to the effects of delayed hits and early accidentals. The delayed-hit probability (Sec. II E 2) predicted by the $\mathrm{MC}$ is compared to data in various regions of the chambers and for different time periods. Residual data-MC differences do not exceed $10 \%$ of the effect, which corresponds to a $\operatorname{Re}\left(\epsilon^{\prime} / \epsilon\right)$ systematic uncertainty of $0.21 \times 10^{-4}$.

There is a component of the early accidental inefficiency that may not be modeled properly because the total TDC range covers only about $2 / 3$ of the relevant early time window that is prior to the in-time window. A systematic error of $0.30 \times 10^{-4}$ is assigned based on the change in $\operatorname{Re}\left(\epsilon^{\prime} / \epsilon\right)$ when accidental hits from only half of the early time window are used to obscure simulated in-time hits. The total uncertainty from modeling the effects of delayed hits and accidentals is $0.37 \times 10^{-4}$ on $\operatorname{Re}\left(\epsilon^{\prime} / \epsilon\right)$.

The modeling of the drift chamber resolutions is checked by comparing the widths of the SOD distributions between data and MC; they agree to within 5\% which corresponds to a systematic error of $0.15 \times 10^{-4}$ on $\operatorname{Re}\left(\epsilon^{\prime} / \epsilon\right)$. 
Apertures: The drift chamber track-separation cut (Fig. 3) depends on the wire spacing, which is known to $20 \mu \mathrm{m}$ on average [32]. The bias in $\operatorname{Re}\left(\epsilon^{\prime} / \epsilon\right)$ from variations in the wire spacing is $(-0.16 \pm 0.12) \times 10^{-4}$ leading to an uncertainty of $0.22 \times 10^{-4}$. The $0.45 \mathrm{~mm}$ uncertainty on the effective regenerator edge (Fig. 7b) leads to a small uncertainty in the expected number of $K_{S}$ decays, and results in a systematic error of $0.20 \times 10^{-4}$ on $\operatorname{Re}\left(\epsilon^{\prime} / \epsilon\right)$.

\section{D. $K \rightarrow \pi^{0} \pi^{0}$ Reconstruction and Selection}

The strategy to identify $K \rightarrow \pi^{0} \pi^{0}$ decays is to reconstruct four photon clusters in the CsI calorimeter that are consistent with coming from two neutral pions, and to reduce background with kinematic cuts. Since the reconstruction of $K \rightarrow \pi^{0} \pi^{0}$ and $K_{L} \rightarrow \pi^{0} \pi^{0} \pi^{0}$ decays is almost identical, most of the discussion will be valid for both these decay modes.

The calorimeter reconstruction begins by determining the energy and position of each cluster found in the Level 2 trigger. The neutral decay mode analysis requires two additional corrections that are not relevant for the $K_{L} \rightarrow \pi^{ \pm} e^{\mp} \nu$ calibration. First, a correction is applied to account for energy shared among nearby clusters. The second correction, described in detail at the end of this section, accounts for a small energy scale difference between data and Monte Carlo.

Each cluster is required to have a transverse energy distribution consistent with that of a photon. This requirement rejects decays that have significant accidental energy overlapping a photon cluster; for $K \rightarrow \pi^{0} \pi^{0}$ decays, it also rejects background from $K_{L} \rightarrow \pi^{0} \pi^{0} \pi^{0}$ decays in which two or more photons overlap at the CsI calorimeter. Sensitivity to modeling the transverse energy distribution is reduced by requiring that all clusters be separated from each other by at least $7.5 \mathrm{~cm}$. To reduce the dependence on the MC trigger simulation, the energy of each cluster is required to be greater than $3 \mathrm{GeV}$.

To reconstruct each neutral pion, we assume that two photon clusters originate from a $\pi^{0}$ decay. In the small angle approximation, the distance between the $\pi^{0}$ decay vertex and the CsI calorimeter is given by

$$
d_{\pi^{0}}=r_{12} \sqrt{E_{\gamma_{1}} E_{\gamma_{2}}} / m_{\pi^{0}},
$$

where $r_{12}$ is the distance between the two photon clusters, $E_{\gamma_{1}}$ and $E_{\gamma_{2}}$ are the two photon energies, and $m_{\pi^{0}}$ is the known mass of the neutral pion. The number of possible photon pairings is 3 for $K \rightarrow \pi^{0} \pi^{0}$ and 15 for $K_{L} \rightarrow \pi^{0} \pi^{0} \pi^{0}$ decays. For each possible photon pairing we compute the quantity

$$
\chi_{\pi^{0}}^{2} \equiv \sum_{j=1}^{N_{\pi^{0}}}\left[\frac{d_{\pi^{0}}^{j}-d_{a v g}}{\sigma_{d}^{j}}\right]^{2},
$$

where $N_{\pi^{0}}$ is the number of neutral pions (2 or 3$), d_{\pi^{0}}^{j}$ is the distance between the CsI and the vertex of the $j^{\prime}$ th $\pi^{0}, d_{\text {avg }}$ is the weighted average of all the $d_{\pi^{0}}^{j}$, and $\sigma_{d}^{j}$ is the energy-dependent $\pi^{0}$-vertex resolution, which is roughly $40 \mathrm{~cm}(30 \mathrm{~cm})$ at the upstream (downstream) end of the decay region. The photon pairing with the minimum $\chi_{\pi^{0}}^{2}$ value is used because this pairing corresponds to the best agreement of the $\pi^{0}$ vertices. To reduce the chance of choosing the wrong photon pairing, we require that the minimum $\chi_{\pi^{0}}^{2}$ value be less than 12 for $K \rightarrow \pi^{0} \pi^{0}$ decays, and less than 24 for $K_{L} \rightarrow \pi^{0} \pi^{0} \pi^{0}$ decays. After all selection cuts, the probability that the minimum $\chi_{\pi^{0}}^{2}$ value gives the wrong pairing is $0.006 \%$ for $K \rightarrow \pi^{0} \pi^{0}$ and $0.02 \%$ for $K_{L} \rightarrow \pi^{0} \pi^{0} \pi^{0}$. The $z$-vertex used in the analysis is given by $Z_{\mathrm{CsI}}-d_{\text {avg }}$, where $Z_{\mathrm{CsI}}$ is the $z$-position of the mean shower depth in the CsI. For $K \rightarrow \pi^{0} \pi^{0}$ decays, the $z$-vertex resolution is $\sim 30 \mathrm{~cm}$ near the regenerator and $\sim 20 \mathrm{~cm}$ near the vacuumwindow.

The beam assignment is made by comparing the $x$ component of the "center-of-energy" to the regenerator $x$ position. The center-of-energy is defined to be

$$
x_{\text {coe }} \equiv \frac{\sum x_{i} E_{i}}{\sum E_{i}}, \quad y_{\text {coe }} \equiv \frac{\sum y_{i} E_{i}}{\sum E_{i}},
$$

where $E_{i}$ are the cluster energies, $x_{i}$ and $y_{i}$ are the cluster coordinates at the CsI calorimeter, and the index $i$ runs over the photons. The center-of-energy is the point at which the kaon would have intercepted the plane of the CsI if it had not decayed. The value of $\left\{x_{\text {coe }}, y_{\text {coe }}\right\}$ typically lies well inside the beam-holes, except for kaons that are scattered by a large angle in either the regenerator or the defining collimator (Sec. IIIF). The centerof-energy resolution is $\sim 1 \mathrm{~mm}$, which is much smaller than the beam separation. An event is assigned to the regenerator beam if $x_{\text {coe }}$ has the same sign as the regenerator $x$-position, or to the vacuum beam if $x_{\text {coe }}$ has the opposite sign.

Significant background rejection comes from the invari-

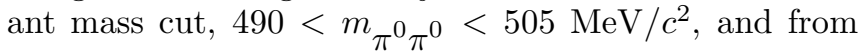
the photon veto cuts. To calculate the invariant mass, the decay vertex is assumed to be on the line joining the target and the center-of-energy, at a distance $d_{\pi^{0}}$ from the calorimeter. Figures $10 \mathrm{c}, \mathrm{d}$ show the invariant mass distributions. In the vacuum beam, events in the mass side-band regions are mostly from $K_{L} \rightarrow \pi^{0} \pi^{0} \pi^{0}$ decays in which two of the six photons are not detected. In the regenerator beam, the side-band regions include comparable contributions from $\pi^{0} \pi^{0}$ pairs produced in the lead of the regenerator (Section IIIF 1), $K \rightarrow \pi^{0} \pi^{0}$ events with the wrong photon pairing, and $K_{L} \rightarrow \pi^{0} \pi^{0} \pi^{0}$ decays.

The photon veto cuts are the same as in the charged mode analysis, with three additional cuts to reduce background: (i) the energy of extra isolated EM clusters is required to be below $0.6 \mathrm{GeV}$ instead of $1.0 \mathrm{GeV}$ in the charged analysis, (ii) the photon-equivalent energy in the beam-hole veto behind the CsI must be less than $5 \mathrm{GeV}$, 
and (iii) the photon-equivalent energy in the Collar Anti that surrounds the CsI beam-holes (Fig. 9) must be less than $1 \mathrm{GeV}$.

Five apertures define the $K \rightarrow \pi^{0} \pi^{0}$ acceptance.

1. The CsI inner aperture near the beam holes is defined by the CA (Fig. 9).

2. The outer CsI aperture is defined by rejecting events in which a photon hits the outer-most layer of crystals (Fig. 5). This cut is applied based on the location of the "seed" crystals that have the maximum energy in each cluster.

3. The upstream aperture in the vacuum beam is defined by the MA (Fig. 8b).

4. The upstream edge in the regenerator beam is defined by the lead at the downstream end of the regenerator (Figure 0 b).

5. The requirement of at least $7.5 \mathrm{~cm}$ between photons results in an effective inner aperture.

Since we do not measure photon angles, the transverse momentum is unknown and therefore cannot be used to reject events in which a kaon scatters in the collimator or regenerator. Instead, we use the center-of-energy (Eq. 9) to define a variable called "Ring Number:"

$$
\mathrm{RING}=40000 \times \operatorname{Max}\left(\Delta x_{\text {coe }}^{2}, \Delta y_{\text {coe }}^{2}\right),
$$

where $\Delta x_{\text {coe }}\left(\Delta y_{\text {coe }}\right)$ is the $x$-distance ( $y$-distance) of the center-of-energy from the center of the closest beam hole. The RING value is the area, in $\mathrm{cm}^{2}$, of the smallest square that is centered on the beam hole and contains the point $\left\{x_{c o e}, y_{c o e}\right\}$. The vacuum and regenerator RING distributions are shown in Fig. 13. The beam size at the CsI is approximately $9 \times 9 \mathrm{~cm}^{2}$, corresponding to RING values less than $81 \mathrm{~cm}^{2}$. The RING distribution between 100 and $150 \mathrm{~cm}^{2}$ is sensitive to scattering in the upstream beryllium absorbers $(z \simeq 19 \mathrm{~m}$, Fig 11). We require RING $<110 \mathrm{~cm}^{2}$, which is a compromise between background reduction and sensitivity to the beam halo simulation.

The final part of the $K \rightarrow \pi^{0} \pi^{0}$ analysis is to match the photon energy scale for data and Monte Carlo. This matching is performed after the background subtraction that will be described in Sec. IIIF. Since the energy scale affects the determination of both the kaon energy and the $z$-vertex position (through Eq. (7), events can migrate into and out of the selected event sample depending on the energy scale. The final energy scale is adjusted to match the data and $\mathrm{MC}$ reconstructed $z$-vertex distribution of $K \rightarrow \pi^{0} \pi^{0}$ decays at the regenerator edge, as shown in Fig. 14a,b. Using $\sim 10^{6}$ events near the regenerator edge, the energy scale is determined in $10 \mathrm{GeV}$ wide kaon energy bins, which is the binning used to extract $\operatorname{Re}\left(\epsilon^{\prime} / \epsilon\right)$. On average, the regenerator edge in data lies $\sim 5 \mathrm{~cm}$ upstream of the MC edge as seen in Fig. 14c; the data-MC
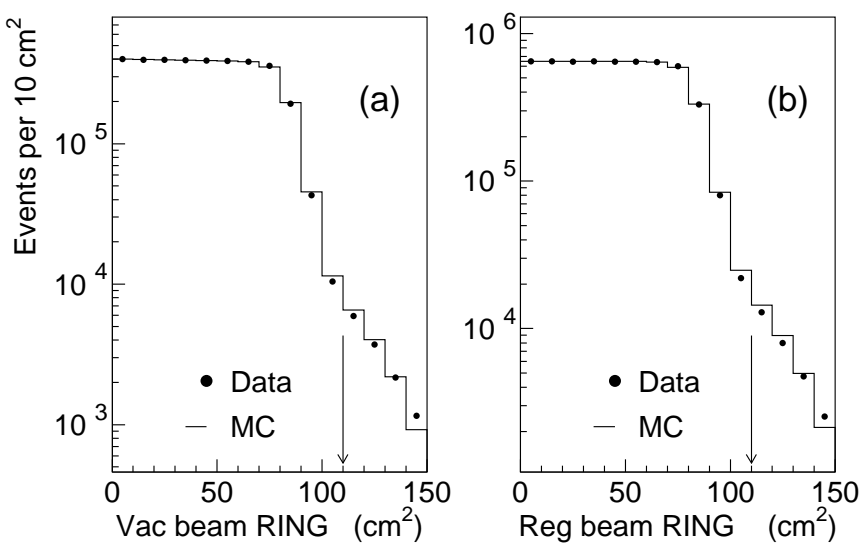

FIG. 13: Vacuum and regenerator RING distributions for $K \rightarrow \pi^{0} \pi^{0}$ events after all other analysis cuts and after background subtraction. The data (MC) are shown by the dots (histogram). The vertical arrows show the signal selection of RING $<110 \mathrm{~cm}^{2}$.

regenerator edge difference varies by a few centimeters depending on the kaon energy.

Since the vertex distance from the CsI is proportional to the cluster energy scale (Eq. 7), the multiplicative energy scale correction is $\sim 0.9992$, which corresponds roughly to the $-5 \mathrm{~cm}$ data-MC difference divided by the 61 meter distance between the regenerator and the CsI. The regenerator edge shift in each $10 \mathrm{GeV}$ kaon energy bin (Fig. 14c) is converted to an energy scale correction, and is applied to each cluster in data.

\section{E. Systematic Uncertainties from $\pi^{0} \pi^{0}$ Trigger, Reconstruction, and Selection}

In this section, we discuss the $\operatorname{Re}\left(\epsilon^{\prime} / \epsilon\right)$ systematic uncertainties from the neutral mode trigger, reconstruction, and selection, which are summarized in Table II. Systematic uncertainties related to background and acceptance are discussed in Sections IIIF 5 and IVA.

Trigger: The Level 1 CsI "energy-sum" trigger is studied using the large $K_{L} \rightarrow \pi^{ \pm} e^{\mp} \nu$ sample in the charged trigger which does not have L1 CsI requirements (Sec. II C 5). The Level 2 cluster-counter is studied using a half-million $K_{L} \rightarrow \pi^{0} \pi^{0} \pi^{0}$ decays from a trigger that requires only Level 1 . The Level 3 systematic uncertainty is based on $1.3 \times 10^{5} K \rightarrow \pi^{0} \pi^{0}$ events that satisfy all analysis cuts and that were accepted online without requiring L3. The combined $\operatorname{Re}\left(\epsilon^{\prime} / \epsilon\right)$ systematic uncertainty from the trigger is $0.18 \times 10^{-4}$.

Cluster Reconstruction: The understanding of the cluster reconstruction in the CsI calorimeter, and in particular the energy scale, results in the largest systematic uncertainty in the $\operatorname{Re}\left(\epsilon^{\prime} / \epsilon\right)$ analysis. After matching the 

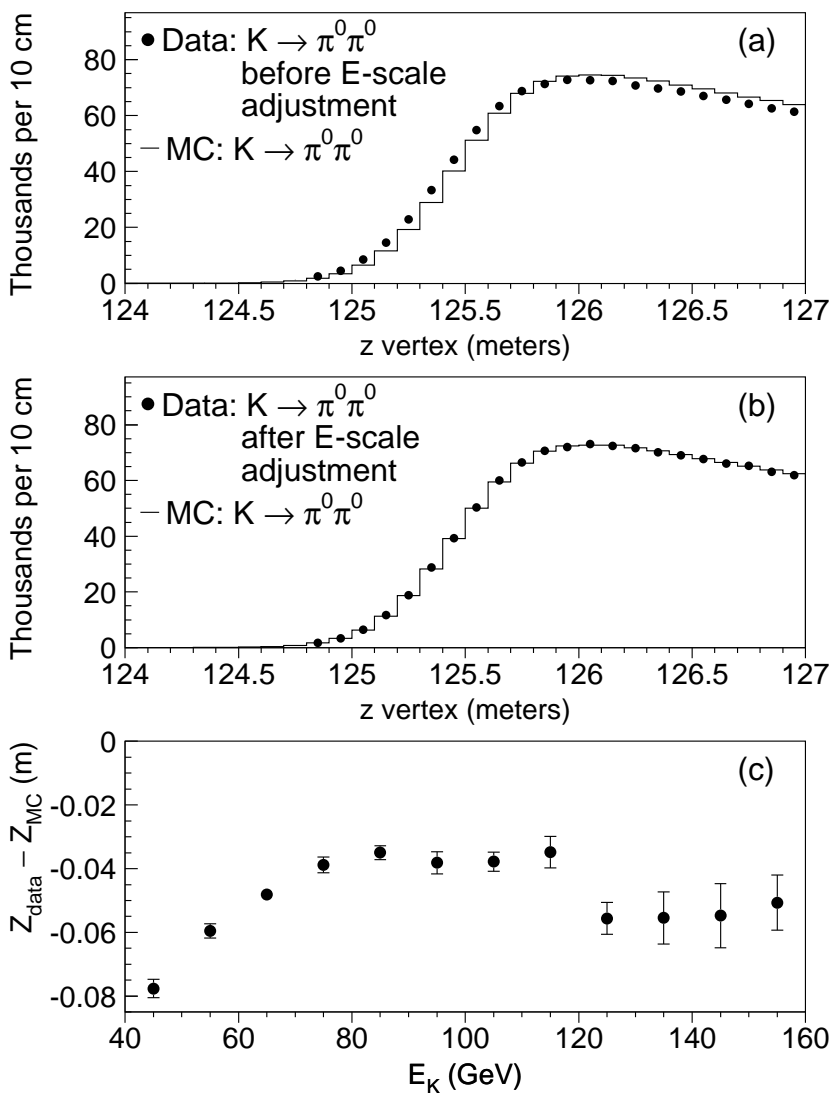

FIG. 14: (a) Reconstructed $K \rightarrow \pi^{0} \pi^{0} z$-vertex near the regenerator edge for data (dots) using the $K_{e 3}$ electron calibration, and for MC (histogram). (b) Same as (a) after the final energy scale adjustment is applied. (c) Data-MC difference in the reconstructed $z$-vertex (meters) in $10 \mathrm{GeV}$ kaon energy bins, before the final energy scale adjustment.

data and $\mathrm{MC}$ at the regenerator edge, we use a variety of other modes that have one or more $\pi^{0} \mathrm{~s}$ in the final state to check how well the data and MC match at other $z$-locations. All of these crosscheck samples are collected at the same time as $K \rightarrow \pi \pi$ decays, and therefore the detector conditions and calibration are precisely the same as for the $K \rightarrow \pi^{0} \pi^{0}$ sample. Data-MC comparisons of the reconstructed neutral vertex, relative to either a charged mode vertex or the known vacuum-window location, are made in the vacuum beam for the following:

1. $K \rightarrow \pi^{0} \pi_{D}^{0}$, where $\pi_{D}^{0}$ refers to $\pi^{0} \rightarrow e^{+} e^{-} \gamma$; the reconstructed $\pi^{0} \rightarrow \gamma \gamma$ vertex is compared to the $e^{+} e^{-} \gamma$ vertex;

2. $K_{L} \rightarrow \pi^{+} \pi^{-} \pi^{0}$ in which the reconstructed $\pi^{0} \rightarrow$ $\gamma \gamma$ vertex is compared to the $\pi^{+} \pi^{-}$vertex;

3. $\eta \rightarrow \pi^{0} \pi^{0} \pi^{0}$, where $\eta$ mesons are produced by beam interactions in the vacuum-window at $z=$ $159 \mathrm{~m}$;

4. $\pi^{0} \pi^{0}$ pairs produced by beam interactions in the vacuum-window at $z=159 \mathrm{~m}$.
TABLE II: Systematic uncertainties in $R e\left(\epsilon^{\prime} / \epsilon\right)$ from the neutral mode trigger and analysis. The uncertainties in the $3 \mathrm{rd}$ column, which also appear in the systematics summary (Table VIII), are the quadratic sum of contributions in the 2nd column.

\begin{tabular}{l|c|c}
\hline \hline Source of & \multicolumn{2}{|c}{$\begin{array}{c}\text { Uncertainty on } R e\left(\epsilon^{\prime} / \epsilon\right) \\
\left(\times 10^{-4}\right)\end{array}$} \\
uncertainty & 0.10 & $\mathbf{0 . 1 8}$ \\
\hline Trigger & 0.13 & \\
L1 trigger & 0.08 & \\
L2 trigger & 1.27 & $\mathbf{1 . 4 7}$ \\
L3 filter & 0.66 & \\
\hline Cluster reconstruction & \\
Energy scale & 0.35 & $\mathbf{0 . 3 7}$ \\
Non-linearity & 0.24 & \\
Position reconstruction & 0.20 & \\
\hline Selection efficiency & 0.20 & $\mathbf{0 . 4 8}$ \\
RING cut & & \\
$\chi_{\pi^{0}}^{2}$ cut & 0.42 & \\
Transverse shape & 0.18 & \\
\hline Apertures & 0.04 & \\
Collar Anti & 0.15 & \\
Mask Anti & - & \\
Reg edge & \\
CsI size & \\
Photon separation &
\end{tabular}

Additional crosschecks at the regenerator edge include $\pi^{0} \pi^{0}$ pairs produced by neutron interactions in the regenerator, and $K^{\star} \rightarrow \pi^{0} K_{S}$ with $K_{S} \rightarrow \pi^{+} \pi^{-}$. A summary of the neutral vertex crosschecks is given in Fig. 15a; they are all consistent with the nominal energy scale correction except for the vacuum-window $\pi^{0} \pi^{0}$ pairs.

Since the vacuum-window $\pi^{0} \pi^{0}$ pairs result in the largest discrepancy, a discussion of the analysis of these events is given here. The selection of vacuum-window $\pi^{0} \pi^{0}$ pairs is similar to that for the $K \rightarrow \pi^{0} \pi^{0}$ decay mode; the main difference is that $K \rightarrow \pi^{0} \pi^{0}$ decays are excluded by selecting events in which the $\pi^{0} \pi^{0}$ invariant mass is at least $15 \mathrm{MeV} / \mathrm{c}^{2}$ away from the kaon mass. The vacuum-window $\pi^{0} \pi^{0}$ sample consists of 45000 events in the vacuum beam. The vacuum-window $z$-location is known with $1 \mathrm{~mm}$ precision using charged two-track events produced in the vacuum-window. The simulation of $\pi^{0} \pi^{0}$ pairs is tuned to match data and MC distributions of reconstructed energy, RING, and $\pi^{0} \pi^{0}$ invariant mass. Figure 15b shows the reconstructed vacuumwindow $\pi^{0} \pi^{0}$ vertex for data and $\mathrm{MC}$ after the regenerator edge $z$-distribution has been matched. The data-MC comparison is complicated by the helium and drift chamber immediately downstream of the vacuumwindow since this extra material is also a source of $\pi^{0} \pi^{0}$ pairs. The production of $\pi^{0} \pi^{0}$ pairs is simulated separately in the vacuum-window, helium, and drift chamber. To evaluate the data-MC discrepancy in the $z$ vertex distribution, a fit is used to determine the relative contribution from each material along with the data-MC vertex shift. The result of this analysis is that the data $z$-vertex distribution at the vacuum-window lies 
$[2.46 \pm 0.16$ (stat) \pm 0.33 (syst) $] \mathrm{cm}$ downstream of the $\mathrm{MC}$ distribution for the 1997 sample; for the 1996 sample, the corresponding shift is $[1.89 \pm 0.24$ (stat) \pm 0.33 (syst) $] \mathrm{cm}$. The data-MC shifts are evaluated separately in the 1996 and 1997 samples because of the different CsI PMT signal integration times.
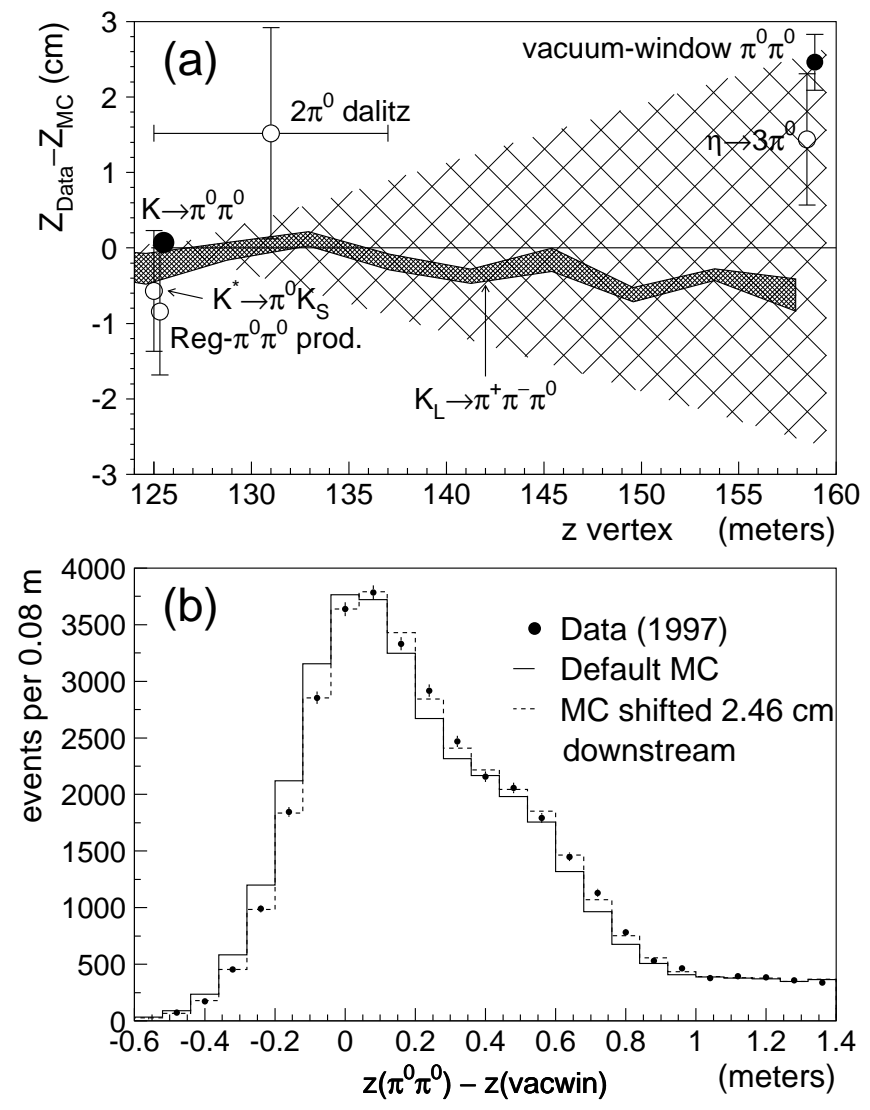

FIG. 15: (a) The difference between the reconstructed data and MC neutral vertex at different locations within the decay region (1997 sample). The $K \rightarrow \pi^{0} \pi^{0}$ mode at $z=125 \mathrm{~m}$ (solid dot) has a data-MC difference of zero by definition since the regenerator edge has been matched. The vacuum-window $\pi^{0} \pi^{0}$ crosscheck at $z=159 \mathrm{~m}$ (solid dot) shows the largest discrepancy; the open circles and $K_{L} \rightarrow \pi^{+} \pi^{-} \pi^{0}$ band show other crosschecks. The hatched region shows the range of discrepancies covered by the assigned systematic uncertainty. (b) The reconstructed $\pi^{0} \pi^{0}$ vertex distribution relative to the vacuum-window for data after matching the regenerator edge (dots), for the default MC (histogram), and for the MC shifted $2.46 \mathrm{~cm}$ downstream (dashed histogram).

The data-MC " $z$-shift" at the vacuum-window is translated into a $\operatorname{Re}\left(\epsilon^{\prime} / \epsilon\right)$ uncertainty by introducing a CsI energy scale distortion to data such that the data and MC $z$-vertex distributions match at both the regenerator and vacuum-window edges; this distortion changes $\operatorname{Re}\left(\epsilon^{\prime} / \epsilon\right)$ by $-1.08 \times 10^{-4}$ and $-1.37 \times 10^{-4}$ for the 1996 and 1997 data samples, respectively. The combined systematic uncertainty on $\operatorname{Re}\left(\epsilon^{\prime} / \epsilon\right)$ is $1.27 \times 10^{-4}$. The energy scale distortion leading to this uncertainty varies linearly with decay vertex in both beams, and corresponds to the hatched region in Fig. 15; non-linear energy scale variations as a function of decay vertex are ruled out because they introduce data-MC discrepancies in other distributions.

Some reconstructed quantities in the analysis do not depend on the CsI energy scale, but are sensitive to energy non-linearities. For example, the $m_{\pi^{0}} \pi^{0}$ peak varies by $0.2 \mathrm{MeV} / \mathrm{c}^{2}$ for kaon energies between 40 and 160 $\mathrm{GeV}$, and the data-MC $z$-difference at the regenerator edge (Fig. 14c) varies by $4 \mathrm{~cm}$ over the same kaon energy range. Such data-MC discrepancies can be reproduced with cluster-energy distortions based on energy, angle, position, and pedestal shift. These distortions are not used in the final result, but are used to determine a systematic uncertainty of $0.66 \times 10^{-4}$ on $\operatorname{Re}\left(\epsilon^{\prime} / \epsilon\right)$.

Cluster position reconstruction is studied using electrons from $K_{e 3}$ decays and comparing the position reconstructed from the CsI to the position of the electron track projected to the mean shower depth in the CsI. The position differences are parameterized and simulated; the maximum $\operatorname{Re}\left(\epsilon^{\prime} / \epsilon\right)$ shift of $0.35 \times 10^{-4}$ is assigned as a systematic uncertainty.

Selection Efficiency: There are three variables for which $\operatorname{Re}\left(\epsilon^{\prime} / \epsilon\right)$ is sensitive to the cut value. (i) Varying the RING cut between 100 and $150 \mathrm{~cm}^{2}$ changes $\operatorname{Re}\left(\epsilon^{\prime} / \epsilon\right)$ by $0.24 \times 10^{-4}$ (Fig. 13). (ii) Relaxing the $\chi_{\pi^{0}}^{2}$ requirement such that the inefficiency from this cut is reduced by a factor of 3 changes $\operatorname{Re}\left(\epsilon^{\prime} / \epsilon\right)$ by $0.20 \times 10^{-4}$. (iii) Removing the transverse energy distribution requirement changes $\operatorname{Re}\left(\epsilon^{\prime} / \epsilon\right)$ by $0.20 \times 10^{-4}$. These three changes added in quadrature contribute an uncertainty of $0.37 \times 10^{-4}$ to $\operatorname{Re}\left(\epsilon^{\prime} / \epsilon\right)$.

Apertures: The aperture uncertainties are mainly from the Collar-Anti (CA, Fig. 9) and Mask-Anti (MA, Fig. 8b). Their effective sizes and positions are measured with $100 \mu m$ precision using $K_{L} \rightarrow \pi^{ \pm} e^{\mp} \nu$ decays, resulting in $\operatorname{Re}\left(\epsilon^{\prime} / \epsilon\right)$ uncertainties of $0.42 \times 10^{-4}$ and $0.18 \times 10^{-4}$ from the CA and MA, respectively. The CsI calorimeter size is known to better than $1 \mathrm{~mm}$ from surveys, resulting in a $0.15 \times 10^{-4}$ uncertainty on $\operatorname{Re}\left(\epsilon^{\prime} / \epsilon\right)$. The $0.1 \mathrm{~mm}$ uncertainty on the effective regenerator edge (Fig. $7 \mathrm{~b}$ ) leads to a $\operatorname{Re}\left(\epsilon^{\prime} / \epsilon\right)$ uncertainty of $0.04 \times 10^{-4}$. Varying the minimum allowed photon separation between $5 \mathrm{~cm}$ and $20 \mathrm{~cm}$ results in no significant change in $\operatorname{Re}\left(\epsilon^{\prime} / \epsilon\right)$. The total $\operatorname{Re}\left(\epsilon^{\prime} / \epsilon\right)$ uncertainty from the neutral mode apertures is $0.48 \times 10^{-4}$.

\section{F. Background to $K \rightarrow \pi^{+} \pi^{-}$and $K \rightarrow \pi^{0} \pi^{0}$}

Two types of background are relevant for this analysis. First, there is "non- $\pi \pi$ " background from misidentification of high branching-ratio decay modes such as semileptonic $K_{e 3}$ and $K_{\mu 3}$ in the charged mode, and $K_{L} \rightarrow \pi^{0} \pi^{0} \pi^{0}$ in the neutral mode. The second type 
of background is from kaons that scatter in the regenerator or the defining collimator and then decay into two pions. Kaon scattering is the same for both the charged and neutral decay modes; it can be largely eliminated in the charged mode analysis using the reconstructed total transverse momentum of the decay products, but the lack of a photon trajectory measurement does not allow a similar reduction in the neutral mode analysis.

Regenerator and collimator scattering affect the $K \rightarrow \pi^{0} \pi^{0}$ reconstruction in two ways. First, a kaon can scatter at a small angle and still reconstruct within the same beam; this "in-beam" background has different regeneration properties and acceptance compared to unscattered kaons, which can cause a bias in $\operatorname{Re}\left(\epsilon^{\prime} / \epsilon\right)$, as well as in kaon parameters such as $\Delta \phi$ and $\Delta m$. Second, a kaon that undergoes large-angle scattering can traverse from one beam to the other beam and then decay, leading to the wrong beam assignment in the neutral mode analysis; this "crossover background" is mostly $K_{S} \rightarrow \pi^{0} \pi^{0}$. Background from regenerator scattering is roughly ten times larger than from scattering in the defining collimator.

All backgrounds are simulated, normalized to data, and then subtracted from the $K \rightarrow \pi \pi$ signal samples. The background-subtraction procedure is described in the following sections, and the background-to-signal ratios are summarized in Table [II].

\section{Non- $\pi \pi$ Background}

Charged pion identification and kinematic cuts (Sec. III B) eliminate most of the charged mode non- $\pi \pi$ background; only a $0.09 \%$ contribution from the semileptonic $K_{\mu 3}$ and $K_{e 3}$ processes remains to be subtracted in the vacuum beam. Both semileptonic background distributions are simulated, and then normalized to data using events reconstructed outside the invariant mass and $p_{T}^{2}$ signal region.

The only substantial non- $\pi \pi$ background in the neutral mode is from $K_{L} \rightarrow \pi^{0} \pi^{0} \pi^{0}$ with undetected photons or photons that have merged at the CsI calorimeter. This $0.11 \%$ background is simulated and then normalized to data using sidebands in the $\pi^{0} \pi^{0}$ invariant mass distribution in the vacuum beam. The decay $K_{L} \rightarrow \pi^{0} \gamma \gamma$ has a branching fraction of $1.7 \times 10^{-6}$ 31; it contributes $2 \times 10^{-5}$ background in the vacuum beam and is ignored. We also ignore $\Xi^{0} \rightarrow \Lambda \pi^{0}$ with $\Lambda \rightarrow n \pi^{0}$, which contributes less than $10^{-5}$ background.

The charged and neutral decay modes both have misidentification background associated with hadronic production in the lead plate of the last regenerator module (Fig. 7b). In both the charged and neutral data samples, this "regenerator-hadron" production is easily isolated in the reconstructed $z$-vertex distribution at the regenerator edge. This background is almost entirely rejected by the $p_{T}^{2}$ cut in the charged decay mode; the remaining $10^{-5}$ background is ignored. In the neutral decay mode, the regenerator $-\pi^{0} \pi^{0}$ background is $8 \times 10^{-5}$ $\left(2 \times 10^{-5}\right)$ in the regenerator (vacuum) beam, and is included in the background simulation.

\section{Collimator Scattering Background}

Scattering in the defining collimators is studied using $K \rightarrow \pi^{+} \pi^{-}$and $K_{L} \rightarrow \pi^{+} \pi^{-} \pi^{0}$ decays in the vacuum beam. Figure 16 shows the $y$ vs. $x$ distribution of the kaon trajectory projected back to the $z$ position of the defining collimator for high $p_{T}^{2}$ vacuum beam events that satisfy all other $K \rightarrow \pi^{+} \pi^{-}$requirements. The square bands show kaons that scattered from the defining collimator edges before decaying. The events in Fig. 16 that lie outside the collimator scattering bands are mainly from semileptonic decays. To determine the number of collimator scatters accurately, the roughly $10 \%$ semileptonic component is subtracted. In the charged decay mode, the background from collimator scattering is only $0.01 \%$, and is small mainly because of the $p_{T}^{2}$ cut; in the neutral decay mode, this background is about $0.1 \%$ and therefore requires an accurate simulation.

The MC simulation propagates each kaon to the defining collimator, and checks if the kaon strikes the collimator at either the upstream end or anywhere along the 3 meter long inner surface. Kaons that hit the collimator are traced through the steel and allowed to scatter back into the beam. A kaon that scatters in the collimator is parameterized to be either pure $K_{S}$ or pure $K_{L}$, with the relative amount adjusted to match the $z$-vertex distribution for the collimator scattering sample shown in Fig. 16.

The MC treatment of collimator scattering is the same in both the vacuum and regenerator beams. About $1 / 3$ of the collimator-scattered kaons hit the Mask Anti (MA, Fig. 8), and can then punch through and exit the MA as either a $K_{L}$ or $K_{S}$. Based on measurements from data, the MC includes a kaon punch-through probability of $60 \%$, and a $K_{S}$ to $K_{L}$ ratio of about 50 for kaons that exit the MA.

\section{Regenerator Scattering Background}

In the charged mode analysis, the regenerator scattering background is $0.074 \%$ in the regenerator beam, and is not present in the vacuum beam. In the neutral mode analysis, the corresponding background levels are $1.13 \%$ in the regenerator beam and $0.25 \%$ in the vacuum beam.

Regenerator scattering is more complicated than collimator scattering, particularly in the time dependence of $K \rightarrow \pi \pi$ decays resulting from the coherent $K_{L}-K_{S}$ mixture. Figure 17 shows the observed proper decay time distributions for kaons that scatter with small $p_{T}^{2}$ $\left(2500-10^{4} \mathrm{MeV}^{2} / c^{2}\right)$ and large $p_{T}^{2}\left(>10^{5} \mathrm{MeV}^{2} / c^{2}\right)$, and for the unscattered $K \rightarrow \pi^{+} \pi^{-}$signal $\left(p_{T}^{2}<\right.$ $\left.250 \mathrm{MeV}^{2} / c^{2}\right)$. Note that the proper time distribution 


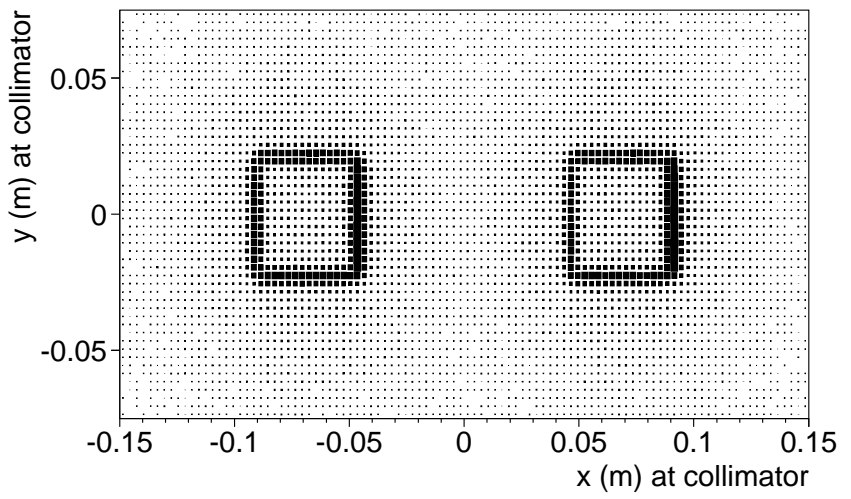

FIG. 16: $y$ vs. $x$ distribution of the kaon trajectory projected back to the $z$ position of the defining collimator in the vacuum beam. The sample shown here is after all $K \rightarrow \pi^{+} \pi^{-}$analysis cuts, except that the $p_{T}^{2}$ cut is changed to $p_{T}^{2}>1000 \mathrm{MeV}^{2} / c^{2}$. The square bands correspond to the edges of the defining collimators.

depends strongly on the $p_{T}^{2}$ value. Large- $p_{T}^{2}$ scattering contributes mainly to crossover background, while small$p_{T}^{2}$ scattering contributes mostly to in-beam background. A detailed description of the regenerator scattering background is needed for the neutral decay mode because the $K \rightarrow \pi^{0} \pi^{0}$ sample includes events with $p_{T}^{2}$ values up to about $3 \times 10^{4} \mathrm{MeV}^{2} / c^{2}$ in the regenerator beam, and up to $5 \times 10^{5} \mathrm{MeV}^{2} / c^{2}$ in the vacuum beam.

A $K \rightarrow \pi \pi$ decay from a kaon that has scattered in the regenerator, referred to as a "regenerator scattering decay," is described in the MC using a function that is fit to acceptance-corrected $K \rightarrow \pi^{+} \pi^{-}$data after subtracting collimator scattering and semileptonic decays. The acceptance correction allows us to fit the "true" regenerator scattering decay distribution, so that the scattering simulation can be used to predict background for both charged and neutral mode decays. As described in Appendix B, the fit function depends on proper time, $p_{T}^{2}$, and kaon momentum. To remove non- $\pi^{+} \pi^{-}$background from charged tracks produced in the regenerator lead (Fig. $7 \mathrm{~b}$ ), the fit excludes decays within $0.2 K_{S}$ lifetimes of the regenerator edge. To avoid the $p_{T}^{2}$ tail from coherent events, only decays with $p_{T}^{2}>2500 \mathrm{MeV}^{2} / c^{2}$ are used in the fit. The fit momentum region is the same as in the signal analysis: 40 to $160 \mathrm{GeV} / c$.

As discussed in Section II C 3, there are two processes that contribute to regenerator scattering. The first process is diffractive scattering, which is identical in the charged and neutral mode analyses because no energy is deposited in the regenerator or photon vetos. The second process is inelastic scattering, which is slighlty different in the two modes because of different photon veto requirements. To address this charged-neutral difference, the fit function is based on a phenomenological model that has separate terms for diffractive and inelastic scattering. The $p_{T}^{2}$ distribution is significantly steeper for diffractive scattering than for inelastic scattering, and this difference allows for the two scattering processes to be distinguished.

Using the fit function to simulate the $K \rightarrow \pi^{0} \pi^{0}$ scattering background, and normalizing to data events in the range $300<$ RING $<800 \mathrm{~cm}^{2}$, we find that the neutral mode veto requirements suppress inelastic scattering by an additional $16 \%$ compared to the charged mode veto requirements. This $16 \%$ charged-neutral difference in the inelastic component corresponds to a $3 \%$ charged-neutral difference in the total regenerator scattering background.

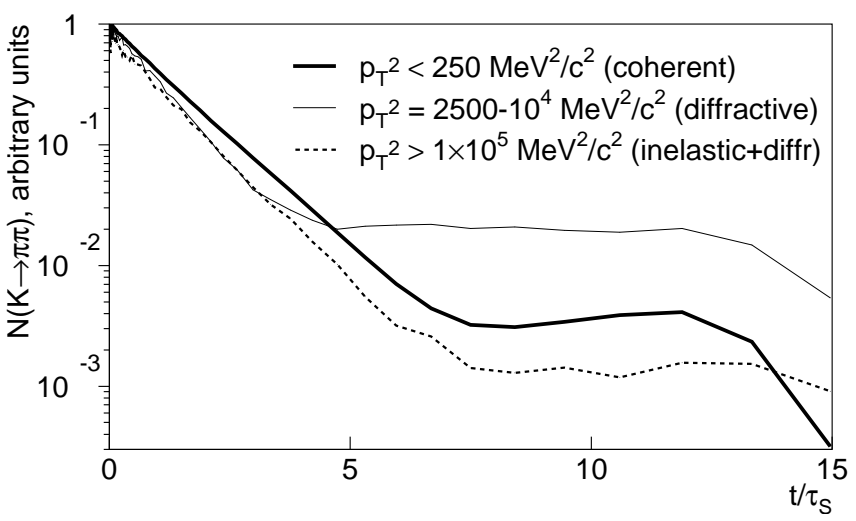

FIG. 17: Acceptance-corrected distribution of the number of regenerator beam $K \rightarrow \pi^{+} \pi^{-}$decays vs. the number of $K_{S}$ lifetimes $\left(t / \tau_{S}\right)$, for kaon momenta between 40 and $50 \mathrm{GeV} / c$. The proper time $t=0$ corresponds to the downstream edge of the regenerator. The $p_{T}^{2}$ ranges are indicated on the plot for each curve. The thick curve is for coherent $K \rightarrow \pi^{+} \pi^{-}$ decays. The thin solid curve corresponds to small $p_{T}^{2}$ and is mostly from diffractive scattering. The dashed curve is for large $p_{T}^{2}$ events, which are due to both inelastic and diffractive scattering. The fluctuations are due to statistics. All $K \rightarrow \pi^{+} \pi^{-}$cuts except $p_{T}^{2}$ have been applied. Backgrounds from collimator scattering and semileptonic decays have been subtracted.

\section{Summary of Backgrounds}

Figure 18 shows the vacuum and regenerator beam $p_{T}^{2}$ distributions after all other charged mode analysis requirements. MC simulations of the background processes are also shown. The regenerator beam background is mostly from regenerator scattering, and the vacuum beam background is mostly from semileptonic decays. Figure 19 shows the neutral mode RING distribution in both beams, along with MC background simulations. Figure 20 shows the neutral mode background-to-signal ratio $(\mathrm{B} / \mathrm{S})$ as a function of the $K \rightarrow \pi^{0} \pi^{0}$ decay vertex. In the regenerator beam, the main background is from regenerator scattering. In the vacuum beam, the largest sources of background are collimator scattering between $110 \mathrm{~m}$ and $125 \mathrm{~m}$, crossover regenerator scattering between $125 \mathrm{~m}$ and $140 \mathrm{~m}$, and $K_{L} \rightarrow \pi^{0} \pi^{0} \pi^{0}$ decays for $z>140 \mathrm{~m}$. 
Table III summarizes the background levels for both decay modes. The charged mode background level is $\sim 10^{-3}$ in both beams; the neutral background level is $1.2 \%$ in the regenerator beam and $0.5 \%$ in the vacuum beam. The background subtraction results in corrections to $\operatorname{Re}\left(\epsilon^{\prime} / \epsilon\right)$ of $-12.5 \times 10^{-4}$ for the neutral decay mode and $-0.2 \times 10^{-4}$ for the charged decay mode.
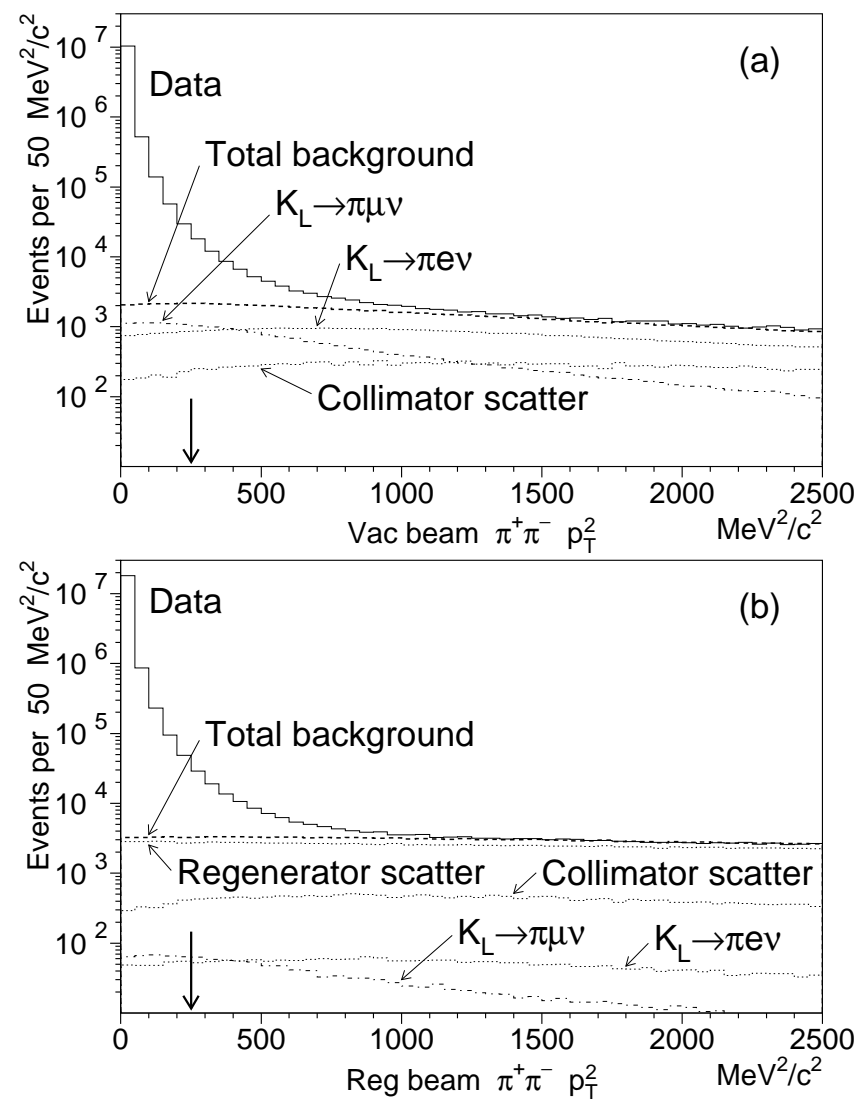

FIG. 18: Data $p_{T}^{2}$ distribution after all other $K \rightarrow \pi^{+} \pi^{-}$ selection cuts for (a) the vacuum beam and (b) the regenerator beam. The Monte Carlo predictions for the background components are overlaid. Events with $p_{T}^{2}<250 \mathrm{MeV}^{2} / c^{2}$ (vertical arrow) are included in the final $K \rightarrow \pi^{+} \pi^{-}$sample.

\section{Systematic Uncertainties from Background}

The uncertainties in $\operatorname{Re}\left(\epsilon^{\prime} / \epsilon\right)$ resulting from the background subtraction are shown in the last column of Table III. In the $K \rightarrow \pi^{+} \pi^{-}$analysis, the background contributes an uncertainty of $0.20 \times 10^{-4}$ on $\operatorname{Re}\left(\epsilon^{\prime} / \epsilon\right)$; this uncertainty is based on changes in $\operatorname{Re}\left(\epsilon^{\prime} / \epsilon\right)$ when background rejection cuts are varied for the $\pi^{+} \pi^{-}$invariant mass, $E / p$, and the minimum pion momentum.

In the $K \rightarrow \pi^{0} \pi^{0}$ analysis, the background contributes an uncertainty of $1.07 \times 10^{-4}$ on $\operatorname{Re}\left(\epsilon^{\prime} / \epsilon\right)$, and is mostly from the $5 \%$ uncertainty on the background level for inbeam regenerator scattering. This background subtrac-
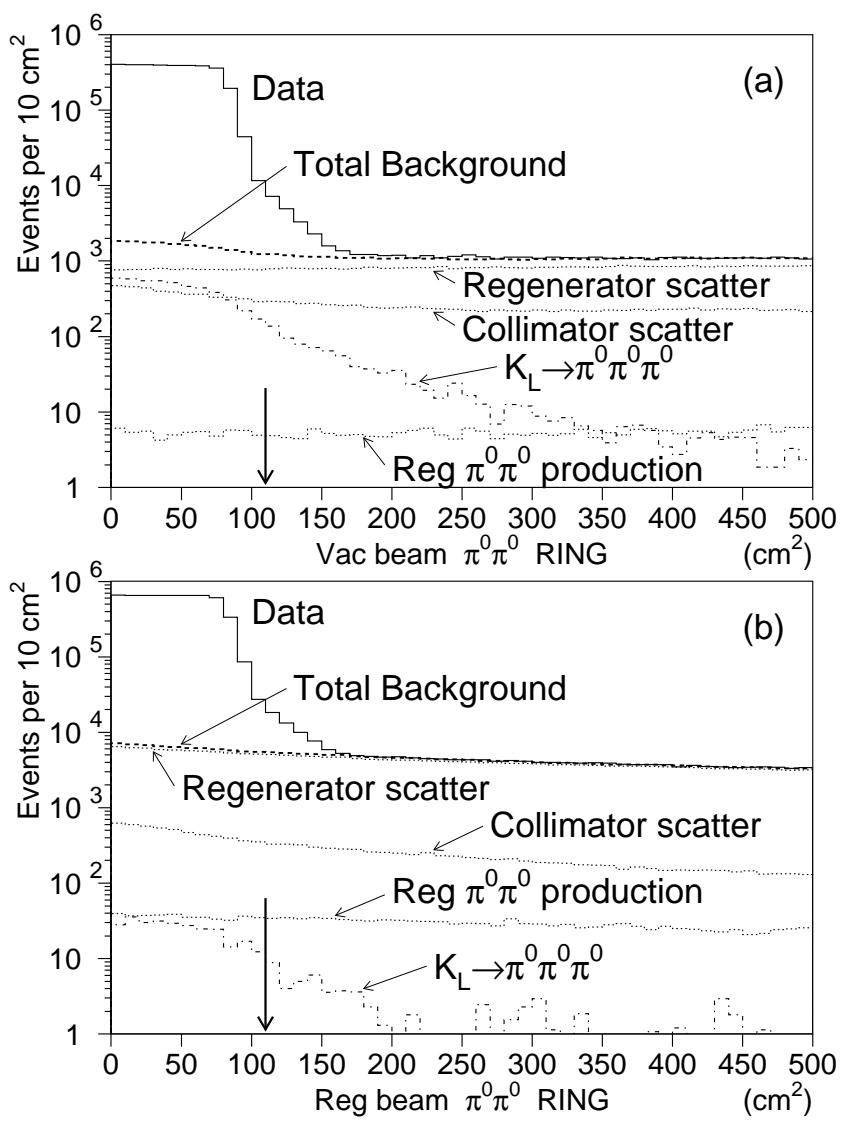

FIG. 19: Data RING distribution after all other $K \rightarrow \pi^{0} \pi^{0}$ selection cuts for (a) the vacuum beam and (b) the regenerator beam. The Monte Carlo predictions for the background components are overlaid. Events with RING $<110 \mathrm{~cm}^{2}$ (vertical arrow) are included in the final $K \rightarrow \pi^{0} \pi^{0}$ sample.

tion depends largely on modeling the acceptance for high $p_{T}^{2} K \rightarrow \pi^{+} \pi^{-}$events.

To check our understanding of the detector acceptance at high $p_{T}^{2}$, we use $\pi^{+} \pi^{-}$pairs from $K_{L} \rightarrow \pi^{+} \pi^{-} \pi^{0}$ decays. Comparing the data and $\mathrm{MC} \pi^{+} \pi^{-} p_{T}^{2}$ distributions, we limit the data-MC difference to be less than $0.5 \%$ per $10000 \mathrm{MeV}^{2} / c^{2}$. To convert this limit on the " $p_{T}^{2}$ slope" into a potential bias on $\operatorname{Re}\left(\epsilon^{\prime} / \epsilon\right)$, we weight the $p_{T}^{2}$ distribution in the neutral mode background simulation by this slope; the resulting $0.4 \times 10^{-4}$ change in $\operatorname{Re}\left(\epsilon^{\prime} / \epsilon\right)$ is included as a systematic uncertainty.

Imperfections in the phenomenological parameterization and fitting of the $K \rightarrow \pi^{+} \pi^{-}$scattering distribution are estimated by comparing charged mode data to the MC simulation. The maximum data-MC difference in the scattering distribution is $3 \%$ of the background level, which corresponds to a $0.50 \times 10^{-4}$ uncertainty for $\operatorname{Re}\left(\epsilon^{\prime} / \epsilon\right)$.

There is also an uncertainty in how the scattering distribution, measured with $K \rightarrow \pi^{+} \pi^{-}$decays, is used to simulate background for $K \rightarrow \pi^{0} \pi^{0}$ decays. As mentioned in Section IIIF 3, the observed charged-neutral difference of $3 \%$ in the regenerator scattering level is ac- 


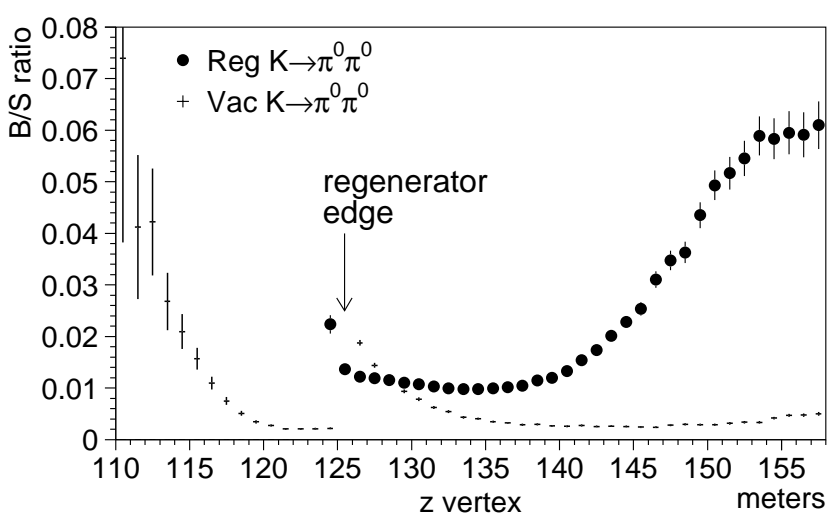

FIG. 20: $\quad K \rightarrow \pi^{0} \pi^{0}$ background-to-signal (B/S) ratio vs. $z$ vertex in 1 meter bins. The regenerator (vacuum) beam is represented by solid dots (crosses). The vertical arrow shows the location of the downstream edge of the regenerator.

TABLE III: The background-to-signal ratio (B/S) for each subtracted background component, for each decay mode, and for each beam. The $\operatorname{Re}\left(\epsilon^{\prime} / \epsilon\right)$ systematic uncertainty associated with each background component is listed in the column labeled " $\sigma_{\text {syst }}$ ".

\begin{tabular}{|c|c|c|c|}
\hline $\begin{array}{l}\text { Background } \\
\text { process }\end{array}$ & $\begin{array}{c}\text { Vac } \\
\text { B/S }(\%)\end{array}$ & $\begin{array}{c}\text { Reg } \\
\text { B/S }(\%)\end{array}$ & $\begin{array}{c}\sigma_{\text {syst }} \\
\left(\times 10^{-4}\right)\end{array}$ \\
\hline \multicolumn{4}{|c|}{$K \rightarrow \pi^{+} \pi^{-}$Analysis: } \\
\hline$K_{e 3}$ & 0.036 & 0.001 & 0.12 \\
\hline$K_{\mu 3}$ & 0.054 & 0.002 & 0.12 \\
\hline Collimator scattering & 0.010 & 0.010 & 0.01 \\
\hline Regenerator scattering & - & 0.074 & 0.10 \\
\hline Total charged & 0.100 & 0.087 & 0.20 \\
\hline \multicolumn{4}{|c|}{ 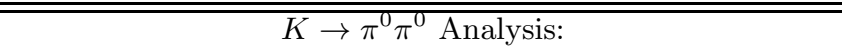 } \\
\hline$K_{L} \rightarrow \pi^{0} \pi^{0} \pi^{0}$ & 0.107 & 0.003 & 0.07 \\
\hline Reg- $\pi^{0} \pi^{0}$ production & 0.002 & 0.008 & 0.05 \\
\hline Collimator scattering & 0.123 & 0.094 & 0.10 \\
\hline Regenerator scattering & 0.252 & 1.130 & 1.06 \\
\hline Total neutral & 0.484 & 1.235 & 1.07 \\
\hline
\end{tabular}

counted for by a $16 \%$ reduction in the inelastic scattering component in the neutral mode background simulation (Appendix B). If we ignore differences between diffractive and inelastic scattering, and simply reduce the total scattering level by $3 \%$ in the neutral mode simulation, $\operatorname{Re}\left(\epsilon^{\prime} / \epsilon\right)$ changes by $+0.3 \times 10^{-4}$. We assign $0.3 \times 10^{-4}$ as the systematic uncertainty on $\operatorname{Re}\left(\epsilon^{\prime} / \epsilon\right)$ to account for the uncertainty in the inelastic-to-diffractive ratio for $K \rightarrow \pi^{0} \pi^{0}$ decays, and to account for possible chargedneutral differences in the $p_{T}^{2}$ distribution.

We have also checked the effect of variations in the analysis requirements on the background subtraction. The most significant effect is from increasing the regenerator-veto threshold from $8 \mathrm{MeV}$ to $24 \mathrm{MeV}$ in both the $K \rightarrow \pi^{+} \pi^{-}$kaon scattering analysis and the $K \rightarrow \pi^{0} \pi^{0}$ signal analysis. This change doubles the inelastic regenerator scattering background and shifts
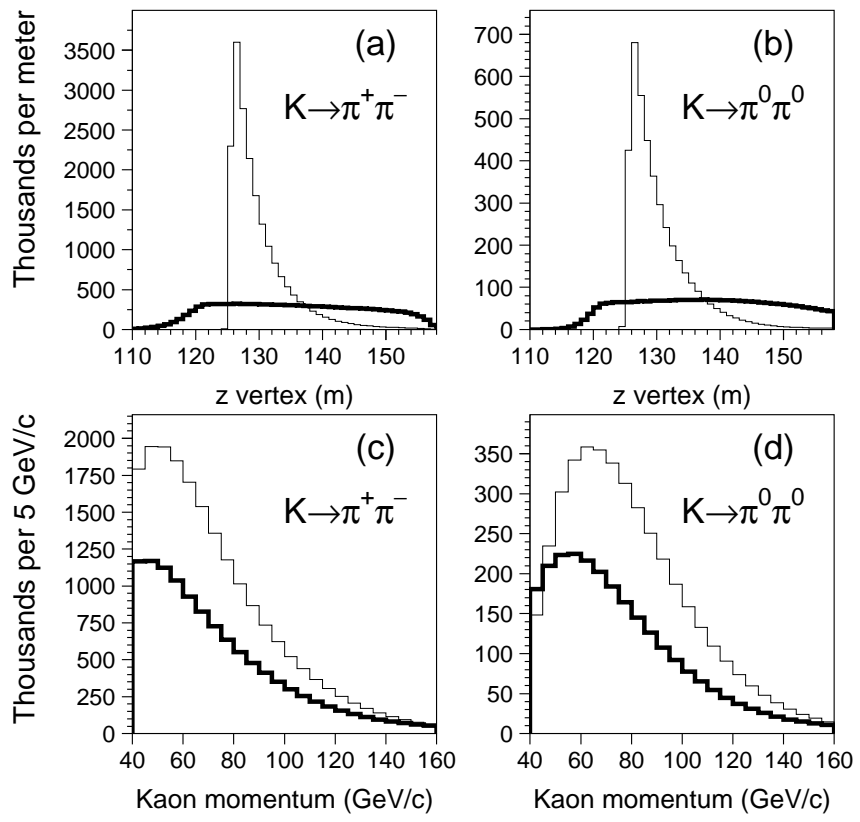

FIG. 21: (a) $z$-vertex distribution for reconstructed $K \rightarrow \pi^{+} \pi^{-}$decays for the vacuum beam (thick) and regenerator beam (thin histogram). (b) $z$-vertex distributions for reconstructed $K \rightarrow \pi^{0} \pi^{0}$ decays. (c) Kaon momentum distributions for reconstructed $K \rightarrow \pi^{+} \pi^{-}$decays. (d) Kaon momentum distributions for reconstructed $K \rightarrow \pi^{0} \pi^{0}$ decays. All $K \rightarrow \pi \pi$ analysis cuts have been applied and background has been subtracted.

$\operatorname{Re}\left(\epsilon^{\prime} / \epsilon\right)$ by $(+0.7 \pm 0.3) \times 10^{-4}$, leading to an additional systematic uncertainty of $0.8 \times 10^{-4}$.

The other neutral mode background sources (Table III) have a much smaller effect on the measurement than regenerator scattering. The $\operatorname{Re}\left(\epsilon^{\prime} / \epsilon\right)$ uncertainty from all neutral mode backgrounds is $1.07 \times 10^{-4}$.

\section{G. Analysis Summary}

The numbers of events after all event selection requirements and background subtraction are given in Table IV. The measurement of $\operatorname{Re}\left(\epsilon^{\prime} / \epsilon\right)$ is statistically limited by the 3.3 million $K_{L} \rightarrow \pi^{0} \pi^{0}$ decays. Figure 21 shows the $z$-vertex and kaon momentum distributions for the four event samples.

TABLE IV: $K \rightarrow \pi \pi$ event totals after all analysis requirements and background subtraction.

\begin{tabular}{l|c|c}
\hline \hline & Vacuum Beam & Regenerator Beam \\
\hline$K \rightarrow \pi^{+} \pi^{-}$ & $11,126,243$ & $19,290,609$ \\
$K \rightarrow \pi^{0} \pi^{0}$ & $3,347,729$ & $5,555,789$ \\
\hline \hline
\end{tabular}




\section{EXTRACTING PHYSICS PARAMETERS}

To measure physics parameters with the event samples described in the previous section, we correct for detector acceptance and perform a fit to the data. The acceptance correction and the associated systematic error are described in Sections IV A IVB. The fitting program used to extract physics results from the event samples is described in Section IV . Systematic uncertainties associated with fitting are discussed in Section IVD.

\section{A. The Acceptance Correction}

A Monte Carlo simulation is used to determine the acceptance, which is the fraction of $K \rightarrow \pi \pi$ decays that satisfy the reconstruction and event selection criteria. The very different $K_{L}$ and $K_{S}$ lifetimes cause a difference between the average acceptance for decays in the two beams. Correcting for the acceptance difference in the momentum and $z$-vertex range used in this analysis, the measured value of $\operatorname{Re}\left(\epsilon^{\prime} / \epsilon\right)$ shifts by $\sim 85 \times 10^{-4}$. About $85 \%$ of this correction is the result of detector geometry, which is known precisely from optical survey and measurements with data. The remaining part of the acceptance correction depends on detailed detector response and resolution in the simulation. Including accidental activity in the simulation results in corrections to $R e\left(\epsilon^{\prime} / \epsilon\right)$ that are about $-0.9 \times 10^{-4}$ and $-0.5 \times 10^{-4}$ for the charged and neutral decay modes, respectively. As will be discussed in Section IV B, comparisons of data and MC $z$-vertex distributions allow us to estimate the systematic uncertainty associated with the acceptance correction.

For a given range of kaon $p$ and $z$, the acceptance is defined as

$$
A_{p, z}=N_{p, z}^{r e c} / N_{p, z}^{g e n},
$$

where $N_{p, z}^{r e c}\left(N_{p, z}^{g e n}\right)$ is the number of reconstructed (generated) Monte Carlo events in the specified $p, z$ range. The generated $p$ and $z$ ranges are slightly larger than the ranges used in the analysis to account for the effects of resolution. Fig. 22 shows the acceptance as a function of $z$ for $70<p<80 \mathrm{GeV} / c$. The $K \rightarrow \pi \pi$ MC samples used to calculate the detector acceptance correspond to 4.7 times the $K \rightarrow \pi^{+} \pi^{-}$data sample and 10.4 times the $K \rightarrow \pi^{0} \pi^{0}$ data sample. The resulting statistical uncertainties on $\operatorname{Re}\left(\epsilon^{\prime} / \epsilon\right)$ from the acceptance correction are $0.41 \times 10^{-4}$ and $0.40 \times 10^{-4}$ for the charged and neutral decay modes, respectively.

As will be described in Section IV , we extract $R e\left(\epsilon^{\prime} / \epsilon\right)$ using twelve $10 \mathrm{GeV} / c$ bins in kaon momentum and a single, integrated $z$ bin. This $p$ binning reduces sensitivity to the momentum dependence of the detector acceptance and to our understanding of the kaon momentum spectrum. The use of $p$ bins also allows us to account for the momentum dependence of the regeneration amplitude.

\section{B. Systematic Uncertainty From The Acceptance Correction}

We evaluate the quality of the simulation by comparing the data and Monte Carlo $z$-vertex distributions in the vacuum beam, where the generated $z$ distribution depends only on the well known $K_{L}$ lifetime. Imperfections in the understanding of detector size and efficiency would change the number of reconstructed events in a non-uniform way along the decay region, and would result in a data-MC difference in the $z$-vertex distribution.

The procedure for converting the data-MC vertex comparison into a systematic uncertainty on $\operatorname{Re}\left(\epsilon^{\prime} / \epsilon\right)$ is as follows. Since $\operatorname{Re}\left(\epsilon^{\prime} / \epsilon\right)$ is measured in $10 \mathrm{GeV} / c$ kaon momentum bins, we weight the number of MC events in each energy bin so that the data and MC kaon momentum distributions agree. We then compare the data and the weighted $\mathrm{MC} z$ distributions, and fit a line to the data/MC ratio as a function of $z$. The slope of this line, $s$, is called an acceptance " $z$-slope." To a good approximation, a $z$-slope affects the measured value of $\operatorname{Re}\left(\epsilon^{\prime} / \epsilon\right)$ as $s \Delta z / 6$, where $\Delta z$ is the difference of the mean $z$ values for the vacuum and regenerator beam vertex distributions, and the factor 6 arises from converting a bias on the vacuum-to-regenerator ratio to a bias on $\operatorname{Re}\left(\epsilon^{\prime} / \epsilon\right) . \Delta z=5.6 \mathrm{~m}$ and $\Delta z=7.2 \mathrm{~m}$ in the charged and neutral $\pi \pi$ modes, respectively. Equation 5 is used to convert the bias on $\operatorname{Re}\left(\epsilon^{\prime} / \epsilon\right)$ to a systematic uncertainty. The uncertainty in $\tau_{L}$ [31], which affects the MC $z$-vertex distribution, contributes a negligible uncertainty of $0.034 \times 10^{-4} \mathrm{~m}^{-1}$ to the $z$-slope.

Figure 23 shows the data-MC $z$-vertex comparisons for the charged and neutral $\pi \pi$ decay modes, and for the high statistics $K_{L} \rightarrow \pi^{ \pm} e^{\mp} \nu$ and $K_{L} \rightarrow \pi^{0} \pi^{0} \pi^{0}$ modes. The $z$-slope in $K_{L} \rightarrow \pi^{+} \pi^{-}$is $(-0.70 \pm 0.30) \times 10^{-4} \mathrm{~m}^{-1}$, and leads to a systematic uncertainty of $0.79 \times 10^{-4}$ in $\operatorname{Re}\left(\epsilon^{\prime} / \epsilon\right)$. This charged $z$-slope has a significance of $2.3 \sigma$, and is mostly from the first $20 \%$ of the data sample (i.e., data collected at the start of the 1997 run). The very small $K_{e 3} z$-slope is shown as a crosscheck, but is not used to set the systematic error because of the different particle types in the final state. To assign a systematic uncertainty for the neutral decay mode acceptance, we use $K_{L} \rightarrow \pi^{0} \pi^{0} \pi^{0}$ decays. This decay mode has the same particle type in the final state as $K \rightarrow \pi^{0} \pi^{0}$ decays, and the $K_{L} \rightarrow \pi^{0} \pi^{0} \pi^{0}$ reconstruction is more sensitive to the effects of nearby clusters, energy leakage at the calorimeter edges, and low photon energies. Using a sample of 50 million reconstructed $K_{L} \rightarrow \pi^{0} \pi^{0} \pi^{0}$ decays in both data and MC, the $z$-slope is $(+0.23 \pm 0.19) \times 10^{-4} \mathrm{~m}^{-1}$ leading to a neutral mode acceptance uncertainty of $0.39 \times 10^{-4}$ on $\operatorname{Re}\left(\epsilon^{\prime} / \epsilon\right)$. The $K \rightarrow \pi^{0} \pi^{0} z$-slope of $(+0.60 \pm 0.53) \times 10^{-4} \mathrm{~m}^{-1}$ is consistent with the $z$-slope in $K_{L} \rightarrow \pi^{0} \pi^{0} \pi^{0}$ decays. The uncertainty in the $z$-dependence of the acceptance for each decay mode is included in the summary of systematic uncertainties shown in Table VIII. 


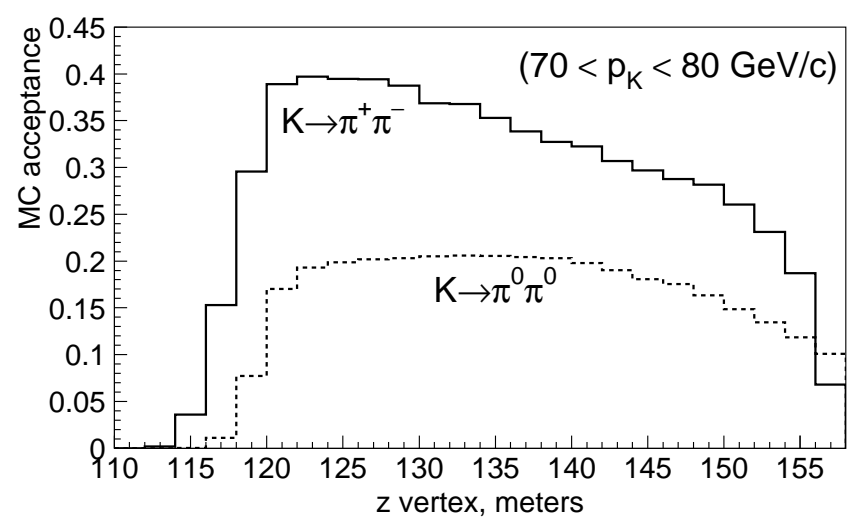

FIG. 22: $\quad$ MC acceptance vs. $z$-vertex (2 meter bins) for $K \rightarrow \pi \pi$ decays with momentum range $70-80 \mathrm{GeV} / c$. The solid (dotted) histogram refers to the charged (neutral) decay mode as indicated on the plot.

\section{Fitting Decay Distributions}

For pure $K_{L}$ and $K_{S}$ beams, the event yields (Table IV]) and acceptance for each mode (Fig. 22) would be sufficient to determine $\operatorname{Re}\left(\epsilon^{\prime} / \epsilon\right)$ from the acceptancecorrected double ratio. The regenerator, however, produces a coherent $K_{S}-K_{L}$ mixture, so that a simple double ratio underestimates $\operatorname{Re}\left(\epsilon^{\prime} / \epsilon\right)$ by a few $\times 10^{-4}$. A proper treatment of the regenerator, as well as target- $K_{S}$ in both beams, is included in a fitting program. In addition to extracting $\operatorname{Re}\left(\epsilon^{\prime} / \epsilon\right)$, this fitting program is also used to make measurements of the kaon parameters $\Delta m$, $\tau_{S}, \phi_{+-}$, and $\Delta \phi$; the fitting procedure described below applies to both $\operatorname{Re}\left(\epsilon^{\prime} / \epsilon\right)$ and these kaon parameters. Each fit has different conditions related to the $z$-binning and CPT assumptions, which are summarized in Table $\mathrm{V}$.

The fitting procedure is to minimize the $\chi^{2}$ between background-subtracted data yields and a prediction function, and uses the MINUIT [33 program. The prediction function $(\mathcal{P})$ for each beam and decay mode is

$$
\mathcal{P}_{p, z}=N_{p, z}^{\pi \pi} \times A_{p, z},
$$

where $N_{p, z}^{\pi \pi}$ is the calculated number of $K \rightarrow \pi \pi$ decays in the specified $p, z$ range, and $A_{p, z}$ is the detector acceptance determined by a Monte Carlo simulation (Eq. 111). Note that $N_{p, z}^{\pi \pi}$ includes full propagation of the kaon state from the target up to the decay point, as in the $\mathrm{MC}$ simulation (Sec. II E 1).

For all fits, the prediction function is computed in $1 \mathrm{GeV} / c p$-bins and 2 meter $z$-bins. To evaluate the $\chi^{2}$ in the $\operatorname{Re}\left(\epsilon^{\prime} / \epsilon\right)$ fit, the event yields and prediction function are integrated in $10 \mathrm{GeV} / c$-wide $p$-bins, and each $p$-bin is integrated over the the full $z$-range from $110 \mathrm{~m}$ to $158 \mathrm{~m}$. For the other kaon parameter fits, the event yields and prediction function are integrated over $10 \mathrm{GeV} / c \times 2 \mathrm{~m}$ $p-z$ bins.

To simplify the discussion that follows, the target- $K_{S}$ component is ignored. For a pure $K_{L}$ beam, the number
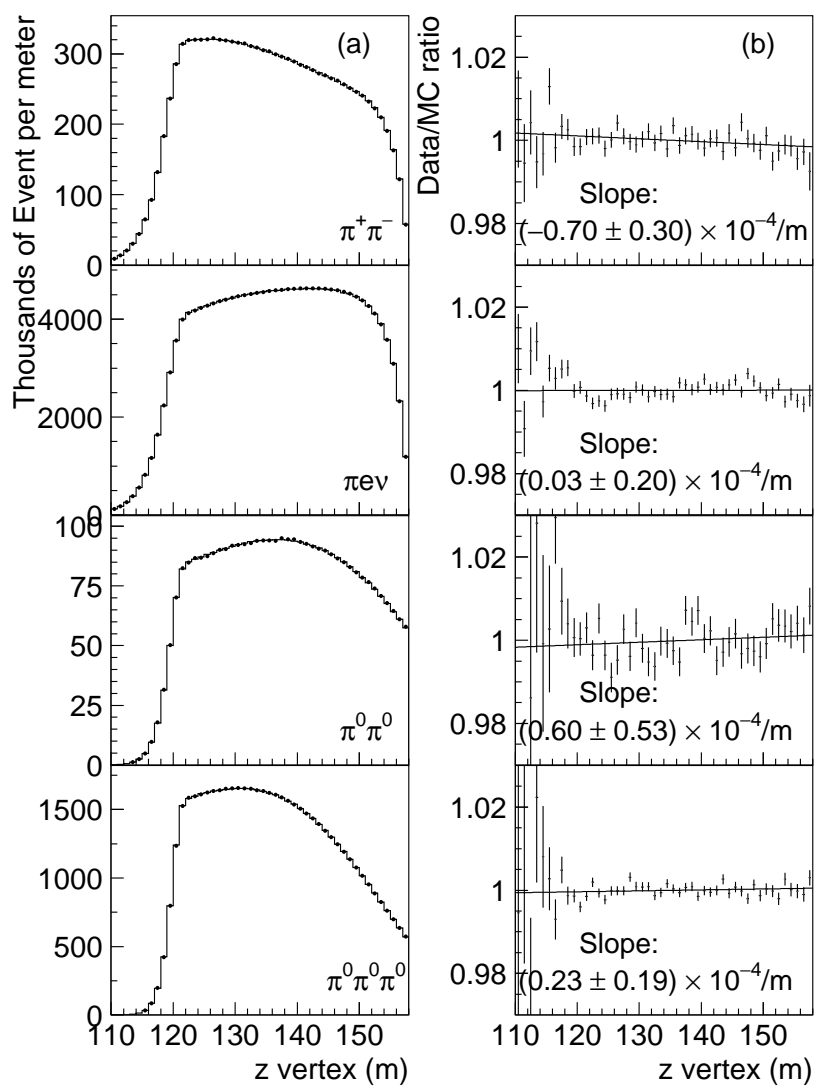

FIG. 23: Comparison of the vacuum beam $z$ distributions for data (dots) and MC (histogram). The data-to-MC ratios on the right are fit to a line, and the $z$-slopes (see text) are shown. The neutral distributions are for the combined $1996+1997$ samples; the charged distributions are for 1997 only.

of $K \rightarrow \pi \pi$ decays is

$$
N_{p, z}^{\pi \pi} \propto \mathcal{F}(p)|\eta|^{2} e^{-t / \tau_{L}},
$$

where $t=m_{K}\left(z-z_{\text {reg }}\right) / p$ is the measured proper time relative to decays at the regenerator edge, $\eta=\eta_{+-}\left(\eta_{00}\right)$ for charged (neutral) decays, $\mathcal{F}(p)$ is the kaon flux, and $\tau_{L}$ is the $K_{L}$ lifetime. The vacuum beam decay distribution is determined by $\tau_{L}$; the total event yield is proportional to $|\eta|^{2}$ and the kaon flux.

For a pure $K_{L}$ beam incident on the $\mathrm{KTeV}$ regenerator, the number of decays downstream of the regenerator is

$$
\begin{array}{r}
N_{p, z}^{\pi \pi} \propto \mathcal{F}_{R}(p) T_{r e g}(p)\left[|\rho(p)|^{2} e^{-t / \tau_{S}}+|\eta|^{2} e^{-t / \tau_{L}}+\right. \\
\left.2|\rho||\eta| \cos \left(\Delta m t+\phi_{\rho}-\phi_{\eta}\right) e^{-t / \tau_{a v g}}\right]
\end{array}
$$

where $\phi_{\eta}=\arg (\eta),|\rho|$ and $\phi_{\rho}$ are the magnitude and phase of the coherent regeneration amplitude [42], $1 / \tau_{\text {avg }} \equiv\left(1 / \tau_{S}+1 / \tau_{L}\right) / 2, \mathcal{F}_{R}(p)$ is the kaon flux upstream of the regenerator, and $T_{\text {reg }}(p)$ is the kaon flux transmission through the regenerator. The prediction function accounts for decays inside the regenerator by using the effective regenerator edge (Fig. $7 \mathrm{~b}$ ) as the start 


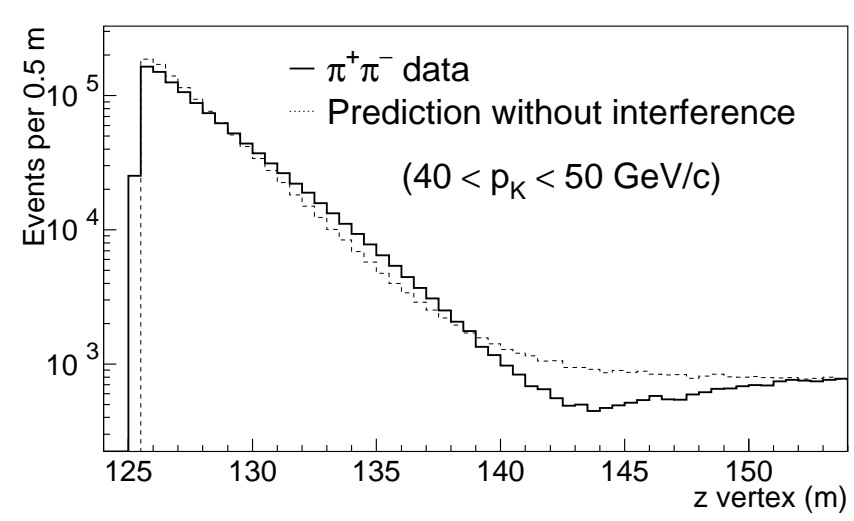

FIG. 24: $z$ decay distribution of $K \rightarrow \pi^{+} \pi^{-}$decays in the regenerator beam, for the restricted momentum range 40$50 \mathrm{GeV} / c$. The MC prediction (dashed) is without the interference term that is proportional to " $2|\rho||\eta| "$ in Eq. 14 .

TABLE V: Fit conditions used to analyze $K \rightarrow \pi \pi$ data. " $z$ binning" refers to using $2 \mathrm{~m} z$-bins in the regenerator beam. "Assume CPT" means that Eq. 15 is a fit constraint. Free parameters common to all fits, but not shown in the table, include the regeneration parameters $\left|f_{-}(70 \mathrm{GeV} / c)\right|$ and $\alpha$, and the kaon flux in each $10 \mathrm{GeV} / c$ momentum bin, $\mathcal{F}\left(p_{1}\right)-$ $\mathcal{F}\left(p_{12}\right)$.

\begin{tabular}{|c|c|c|c|}
\hline \multirow[b]{2}{*}{$\begin{array}{l}\text { Fit } \\
\text { Type }\end{array}$} & \multicolumn{3}{|c|}{ Fit Conditions } \\
\hline & $z$ binning & $\begin{array}{l}\text { Assume } \\
\text { CPT }\end{array}$ & $\begin{array}{l}\text { Free } \\
\text { Parameters }\end{array}$ \\
\hline$\overline{R e}\left(\epsilon^{\prime} / \epsilon\right)$ & No & $\overline{\text { Yes }}$ & $\overline{R e}\left(\epsilon^{\prime} / \epsilon\right)$ \\
\hline$\Delta m, \tau_{S}$ & Yes & Yes & $\Delta m, \tau_{S}$ \\
\hline$\phi_{+-}$ & Yes & No & $\Delta m, \tau_{S}, \phi_{+-}$ \\
\hline$\Delta \phi$ & Yes & No & $\begin{array}{l}\Delta m, \tau_{S}, \phi_{+-} \\
\operatorname{Re}\left(\epsilon^{\prime} / \epsilon\right), \operatorname{Im}\left(\epsilon^{\prime} / \epsilon\right)\end{array}$ \\
\hline
\end{tabular}

of the decay region. All three terms in Eq. 14 are important, as illustrated in Fig. 24, which shows interference effects in the regenerator-beam $z$-vertex distribution.

Next, we discuss how the various factors in Eqs. 13 14 are treated in the fits. The average vacuum-toregenerator kaon flux ratio $\left(\mathcal{F} / \mathcal{F}_{R}\right)$ and the average regenerator transmission $\left(T_{\text {reg }}\right)$ cancel in the $R e\left(\epsilon^{\prime} / \epsilon\right)$ fit as explained in Appendix A. To account for the momentum dependence of the regeneration amplitude in the fits (see below), we need to know the momentumdependence of $\mathcal{F} /\left(\mathcal{F}_{R} T_{\text {reg }}\right)$; it is measured from the vacuum-to-regenerator ratio of $K_{L} \rightarrow \pi^{+} \pi^{-} \pi^{0}$ decays, and is found to vary linearly by $(+7.0 \pm 0.7) \%$ between 40 and $160 \mathrm{GeV} / c$. This variation in $\mathcal{F} /\left(\mathcal{F}_{R} T_{\text {reg }}\right)$ is mostly from the momentum dependence of the regenerator transmission, and to a lesser extent from the movable absorber transmission.

The $K_{L}$ lifetime is taken from [31]. The values of $\Delta m$ and $\tau_{S}$ are fixed to our measurements (Sec. VIA) for the $\operatorname{Re}\left(\epsilon^{\prime} / \epsilon\right)$ fit, and are floated in the fits for the other kaon parameters. In the fits that assume CPT symmetry, $\phi_{+-}$ and $\phi_{00}$ are set equal to the superweak phase:

$$
\phi_{\eta}=\phi_{S W}=\tan ^{-1}(2 \Delta m / \Delta \Gamma) .
$$

The final component of the prediction function is the regeneration amplitude. We use a model that relates $\rho$ to the difference between the forward kaon-nucleon scattering amplitudes for $K^{0}$ and $\overline{K^{0}}$ :

$$
\rho \propto f_{-} \equiv \hbar \frac{f(0)-\bar{f}(0)}{p},
$$

where $f(0)$ and $\bar{f}(0)$ are the forward scattering amplitudes for $K^{0}$ and $\overline{K^{0}}$, respectively, and $p$ is the kaon momentum. Additional factors that contribute to $\rho$ are described in 34.

For an isoscalar target and high kaon momentum, $f_{-}$ can be approximated by a single Regge trajectory corresponding to the $\omega$ meson. In that case, Regge theory 35 predicts that the magnitude of $f_{-}$should vary with kaon momentum as a power law:

$$
\left|f_{-}(p)\right|=\left|f_{-}(70 \mathrm{GeV} / c)\right|\left(\frac{p}{70 \mathrm{GeV} / c}\right)^{\alpha} .
$$

The complex phase of $f_{-}$can be determined from its momentum dependence through an integral dispersion relation, with the requirement that the forward scattering amplitudes be analytic functions. This "analyticity" requirement yields a constant phase for a power-law momentum dependence:

$$
\phi_{f_{-}}=-\frac{\pi}{2}(2+\alpha)
$$

In practice, the kaon-nucleon interactions in carbon are screened due to rescattering processes. The effects of screening modify the momentum dependence of $\left|f_{-}(p)\right|$ as well as its phase. Screening corrections are evaluated using Glauber theory formalism [36, 37] for diffractive scattering, and using various models [38 for inelastic scattering. The screening corrections in the prediction function used in the $\operatorname{Re}\left(\epsilon^{\prime} / \epsilon\right)$ fit result in a $10 \%$ correction to $\alpha$, and a $0.36 \times 10^{-4}$ shift in $\operatorname{Re}\left(\epsilon^{\prime} / \epsilon\right)$.

\section{Systematic Uncertainties from Fitting}

Uncertainties from the fitting procedure are summarized in Table VI and discussed below. These uncertainties are mainly related to regenerator properties, and contribute $0.30 \times 10^{-4}$ to the $\operatorname{Re}\left(\epsilon^{\prime} / \epsilon\right)$ uncertainty.

The uncertainty on the momentum dependence of the regenerator transmission corresponds to a $0.19 \times 10^{-4}$ uncertainty on $\operatorname{Re}\left(\epsilon^{\prime} / \epsilon\right)$. The sensitivity to target- $K_{S}$ is checked by floating the $K^{0} / \overline{K^{0}}$ flux ratio (Sec. IIE 1 ) in the fit; this changes the target- $K_{S}$ component by $(2.5 \pm$ $1.6) \%$ of itself, and leads to a systematic uncertainty of $0.12 \times 10^{-4}$ on $\operatorname{Re}\left(\epsilon^{\prime} / \epsilon\right)$. 
TABLE VI: Systematic uncertainties in $R e\left(\epsilon^{\prime} / \epsilon\right)$ from fitting.

\begin{tabular}{lc}
\hline \hline Source of Uncertainty & $\begin{array}{r}\operatorname{Re}\left(\epsilon^{\prime} / \epsilon\right) \text { Uncertainty } \\
\left(\times 10^{-4}\right)\end{array}$ \\
\hline Regenerator transmission & 0.19 \\
Target- $K_{S}$ & 0.12 \\
$\Delta m$ and $\tau_{S}$ & 0.11 \\
Regenerator screening & 0.15 \\
$\phi_{\rho}$ (analyticity) & 0.07 \\
1996 vs. $1997 K_{S} / K_{L}$ flux ratio & 0.05 \\
$\tau_{L}[31]$ & 0.02 \\
\hline Total & 0.30 \\
\hline \hline
\end{tabular}

The dependence of $\operatorname{Re}\left(\epsilon^{\prime} / \epsilon\right)$ on $\Delta m$ and $\tau_{S}$ is

$$
\begin{aligned}
\Delta R e\left(\epsilon^{\prime} / \epsilon\right)= & \left(+0.05 \times 10^{-4}\right) \times \frac{\Delta m-5261}{15} \\
& +\left(-0.06 \times 10^{-4}\right) \times \frac{\tau_{S}-89.65}{0.07},
\end{aligned}
$$

where $\Delta m$ and $\tau_{S}$ are in units of $10^{6} \hbar \mathrm{s}^{-1}$ and $10^{-12} \mathrm{~s}$, respectively. Each numerator in Eq. 19 is the difference between the true value and the $\mathrm{KTeV}$ measurement (Sec. VI A); the denominators are the total KTeV uncertainties on $\Delta m$ and $\tau_{S}$. Since the $\mathrm{KTeV}$ measurements of $\Delta m$ and $\tau_{S}$ are anti-correlated, the systematic uncertainty on $\operatorname{Re}\left(\epsilon^{\prime} / \epsilon\right)$ due to variations in these parameters is $0.11 \times 10^{-4}$.

There are also uncertainties in $\operatorname{Re}\left(\epsilon^{\prime} / \epsilon\right)$ associated with the analyticity relation and screening correction used to predict the regeneration phase $\phi_{\rho}$. It has been argued that the analyticity assumption is good to $0.35^{\circ}$ in the E773 experiment 39], which included kaon momenta down to $30 \mathrm{GeV} / c$. A smaller deviation from analyticity is expected with the $40 \mathrm{GeV} / c$ minimum momentum cut used in this analysis; this leads to a $0.25^{\circ}$ uncertainty in $\phi_{\rho}$ and a $0.07 \times 10^{-4}$ uncertainty in $\operatorname{Re}\left(\epsilon^{\prime} / \epsilon\right)$. Using different screening models in the fit leads to a $\operatorname{Re}\left(\epsilon^{\prime} / \epsilon\right)$ uncertainty of $0.15 \times 10^{-4}$.

The fitting program uses the same $K_{S} / K_{L}$ flux ratio for the charged and neutral decay modes. Since the 1996 $K \rightarrow \pi^{+} \pi^{-}$sample is excluded, we consider the possibility of a change in the kaon flux ratio between 1996 and 1997. The kaon flux ratio depends only on the physical properties of the movable absorber and regenerator. The density of these two elements could change between the two years because of a possible few degree temperature difference, leading to a systematic uncertainty of $0.05 \times 10^{-4}$ on $\operatorname{Re}\left(\epsilon^{\prime} / \epsilon\right)$.

Although fitting uncertainties in the $R e\left(\epsilon^{\prime} / \epsilon\right)$ measurement are a small part of the total uncertainty, they are more significant in the measurements of $\Delta m, \tau_{S}$, and $\phi_{+-}$(Table VII . Uncertainties in the regenerator transmission and in the analyticity assumption contribute the largest uncertainties in the measurements of $\Delta m$ and $\tau_{S}$. The uncertainty in the regenerator screening model
TABLE VII: Fitting uncertainties in $\Delta m, \tau_{S}, \phi_{+-}$, and $\Delta \phi$.

\begin{tabular}{|c|c|c|c|c|}
\hline \multirow[b]{2}{*}{$\begin{array}{l}\text { Source of } \\
\text { Uncertainty }\end{array}$} & \multicolumn{4}{|c|}{$\sigma_{\text {syst }}$ for: } \\
\hline & $\begin{array}{c}\Delta m \\
\left(\times 10^{6} \hbar / s\right)\end{array}$ & $\begin{array}{c}\tau_{S} \\
\left(\times 10^{-12} \mathrm{~s}\right)\end{array}$ & $\begin{array}{l}\phi_{+-} \\
\left({ }^{\circ}\right)\end{array}$ & $\begin{array}{l}\Delta \phi \\
\left({ }^{\circ}\right)\end{array}$ \\
\hline Regen. transmission & 10.0 & 0.020 & 0.07 & 0.01 \\
\hline Target- $K_{S}$ & 1.4 & 0.017 & 0.13 & 0.01 \\
\hline Regen. screening & 3.0 & 0.020 & 0.75 & 0.03 \\
\hline$\phi_{\rho}$ (analyticity) & 8.1 & 0.030 & 0.25 & 0.00 \\
\hline$\tau_{L}$ & 0.0 & 0.001 & 0.00 & 0.00 \\
\hline Total & 13.3 & 0.045 & 0.80 & 0.03 \\
\hline
\end{tabular}
" $\sigma_{\text {syst }}$ " refers to systematic uncertainty.

contributes an uncertainty of $0.75^{\circ}$ in the $\phi_{+-}$measurement. Fitting uncertainties have a negligible effect on the measurement of $\Delta \phi$ because of cancellations between the charged and neutral decay modes.

\section{MEASUREMENT OF $R e\left(\epsilon^{\prime} / \epsilon\right)$}

The KTeV measurement of $\operatorname{Re}\left(\epsilon^{\prime} / \epsilon\right)$ uses the background-subtracted $K \rightarrow \pi^{+} \pi^{-}$and $K \rightarrow \pi^{0} \pi^{0}$ samples in the vacuum and regenerator beams, the prediction for the $K \rightarrow \pi \pi$ acceptances using the Monte Carlo, and the fitting program. Section VA presents the $R e\left(\epsilon^{\prime} / \epsilon\right)$ result and a summary of the systematic uncertainties. Section VB presents several crosschecks, including a "reweighting" technique which does not use a Monte Carlo acceptance correction.

\section{A. The $R e\left(\epsilon^{\prime} / \epsilon\right)$ Result}

There are 48 measured quantities that enter into the $R e\left(\epsilon^{\prime} / \epsilon\right)$ fit: the observed numbers of $K \rightarrow \pi^{+} \pi^{-}$and $K \rightarrow \pi^{0} \pi^{0}$ decays in the vacuum and regenerator beams, each in twelve $10 \mathrm{GeV} / c$ wide $p$ bins. Within each momentum bin we use the $z$-integrated yield from $110 \mathrm{~m}$ to $158 \mathrm{~m}$. There are 27 fit parameters including 24 kaon fluxes, two regeneration parameters, and $\operatorname{Re}\left(\epsilon^{\prime} / \epsilon\right)$. Therefore, the number of degrees of freedom in the fit is $48-27=21$. CPT symmetry is assumed (Eq. 15), and the values of $\Delta m$ and $\tau_{S}$ are from our measurements described in Sec. VIA.

For the combined 1996 and 1997 datasets, we obtain

$$
\begin{array}{ccc}
\operatorname{Re}\left(\epsilon^{\prime} / \epsilon\right) & = & (20.71 \pm 1.48) \times 10^{-4} \\
\left|f_{-}(70 \mathrm{GeV} / c)\right| & = & 1.2070 \pm 0.0003 \text { mbarns } \\
\alpha & = & -0.5426 \pm 0.0008 \\
\chi^{2} / \text { dof } & = & 27.6 / 21
\end{array}
$$

where the errors reflect the statistical uncertainties. Including the systematic uncertainty,

$$
\begin{aligned}
\operatorname{Re}\left(\epsilon^{\prime} / \epsilon\right) & =[20.71 \pm 1.48(\text { stat }) \pm 2.39 \text { (syst) }] \times 10^{-4} \\
& =(20.7 \pm 2.8) \times 10^{-4}
\end{aligned}
$$


TABLE VIII: Summary of systematic uncertainties in $R e\left(\epsilon^{\prime} / \epsilon\right)$. Uncertainties from the charged and neutral decay modes are presented in more detail in Tables If and II.

\begin{tabular}{lcc}
\hline \hline & \multicolumn{2}{c}{$\operatorname{Re}\left(\epsilon^{\prime} / \epsilon\right)$} \\
Source of uncertainty & $K \rightarrow \pi^{+} \pi^{-}$ & $K \rightarrow \pi^{0} \pi^{0}$ \\
from: & 0.18 \\
Trigger & 0.58 & 1.47 \\
CsI energy, position recon & - & - \\
Track reconstruction & 0.32 & 0.37 \\
Selection efficiency & 0.47 & 0.48 \\
Apertures & 0.30 & 1.07 \\
Background & 0.20 & 0.39 \\
z-dependence of acceptance & 0.79 & 0.40 \\
MC statistics & 0.41 & 0.30 \\
Fitting & \multicolumn{2}{c}{2.39} \\
\hline TOTAL & \multicolumn{2}{c}{} \\
\hline \hline
\end{tabular}

where the contributions to the systematic uncertainty are summarized in Table VIII. The systematic uncertainties from the charged and neutral decay modes contribute $1.26 \times 10^{-4}$ and $2.00 \times 10^{-4}$, respectively. The largest uncertainties are from the CsI energy reconstruction $\left(1.47 \times 10^{-4}\right)$, neutral mode background subtraction $\left(1.07 \times 10^{-4}\right), z$-dependence of the acceptance in the charged decay mode $\left(0.79 \times 10^{-4}\right)$, and the charged mode Level 3 filter $\left(0.54 \times 10^{-4}\right)$.

\section{B. $\operatorname{Re}\left(\epsilon^{\prime} / \epsilon\right)$ Crosschecks}

\section{Consistency Among Data Subsets}

We have performed several crosschecks of our result by dividing the $K \rightarrow \pi \pi$ samples into subsets and checking the consistency of $\operatorname{Re}\left(\epsilon^{\prime} / \epsilon\right)$ and other parameters among the different subsets. Figure 25 shows the $\operatorname{Re}\left(\epsilon^{\prime} / \epsilon\right)$ result in roughly month-long time periods, in each regenerator position, and for the two magnet polarities. These comparisons all show good agreement. The first data point labeled $96 / 97$ a corresponds to the current analysis applied to the sample used in our previous publication [20; this reanalysis is discussed in Appendix G.

To check the dependence on kaon momentum, 12 separate fits are done in $10 \mathrm{GeV} / c$ momentum bins. The free parameters in each fit are $\operatorname{Re}\left(\epsilon^{\prime} / \epsilon\right)$ and $\left|f_{-}(70 \mathrm{GeV} / c)\right|$, which is proportional to the regeneration amplitude at $70 \mathrm{GeV} / c$. The momentum dependence of the regeneration amplitude within each $10 \mathrm{GeV} / c$ bin is described by the power-law, $\rho \sim p^{\alpha}$, where $\alpha$ is fixed to the value found in the nominal fit (Eq. 20). The $\chi^{2}$ per degree of freedom is $20.7 / 11$ for $\operatorname{Re}\left(\epsilon^{\prime} / \epsilon\right)$ vs. $p$ (Fig. 26a) and 5.2/11 for $\left|f_{-}(70 \mathrm{GeV} / c)\right|$ vs. $p$ (Fig. 26 b); the combined $\chi^{2} /$ dof is $25.9 / 22$. The scatter of $\operatorname{Re}\left(\epsilon^{\prime} / \epsilon\right)$ in the higher momentum bins is not present in the kaon parameter measurements (Fig. 28), and a linear fit to $\operatorname{Re}\left(\epsilon^{\prime} / \epsilon\right)$ vs. $p$ has no significant slope $(<0.8 \sigma)$. The $140-150 \mathrm{GeV} / c$ bin, which accounts for 6.3 of the $\chi^{2}$ in Fig. 26a, also contributes 6.7

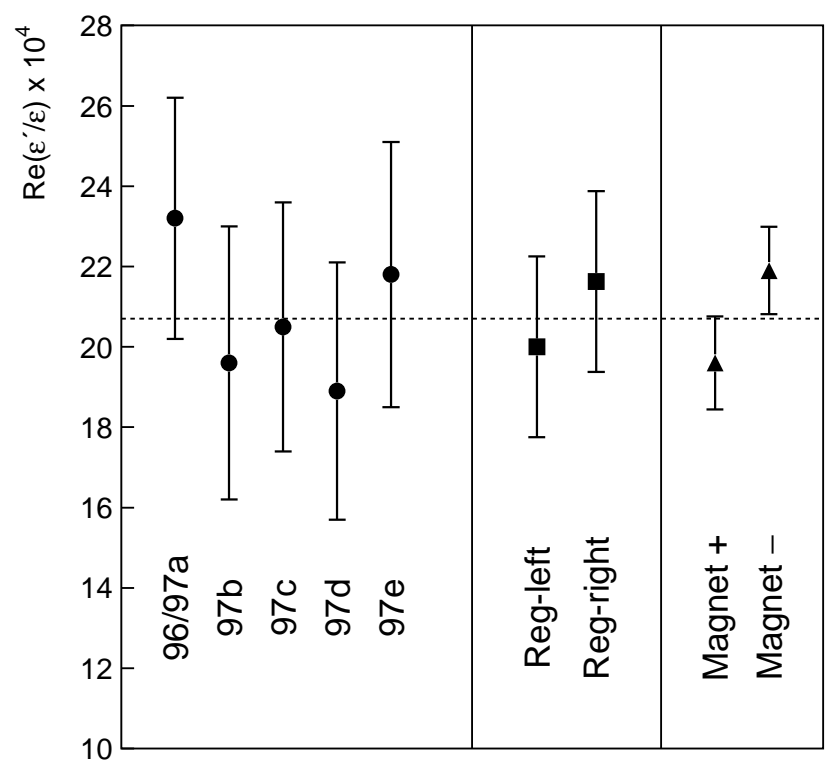

FIG. 25: $\operatorname{Re}\left(\epsilon^{\prime} / \epsilon\right)$ consistency checks. The first five data points correspond roughly to one-month time periods. RegLeft and Reg-Right correspond to the two regenerator positions. Magnet+ and Magnet- correspond to the two magnet polarities, with the $K \rightarrow \pi^{0} \pi^{0}$ sample common to both results. The uncertainties shown within each category are independent statistical errors.

to the $\chi^{2}$ in the nominal fit (Eq. 20), with nearly equal contributions from all four $K \rightarrow \pi \pi$ samples.

Another crosscheck is that $\alpha$, which describes the regeneration power law (Eq. 17), should be the same for both the charged and neutral decays. A separate fit in each decay mode results in

$$
\begin{gathered}
\alpha_{+-}=-0.5421 \pm 0.0009 \text { (stat) } \\
\alpha_{00}=-0.5445 \pm 0.0017 \text { (stat) }
\end{gathered}
$$

which agree to within $1.2 \sigma$.

The target- $K_{S}$ correction is checked in a separate $R e\left(\epsilon^{\prime} / \epsilon\right)$ fit that uses only those events with kaon momenta below $100 \mathrm{GeV} / c$ and a $z$-vertex farther than $124 \mathrm{~m}$ from the $\mathrm{BeO}$ target. This sample has a negligible $K_{S}$ component, and is therefore described by essentially pure $K_{L}$ beams entering the decay region. Using this sub-sample, the change in $R e\left(\epsilon^{\prime} / \epsilon\right)$ from the nominal result (Eq. 21) is $(+0.85 \pm 0.89) \times 10^{-4}$, where the error reflects the uncorrelated statistical uncertainty.

\section{2. $\operatorname{Re}\left(\epsilon^{\prime} / \epsilon\right)$ From Reweighting Technique}

As a final crosscheck of our "standard" analysis, we also measure $\operatorname{Re}\left(\epsilon^{\prime} / \epsilon\right)$ using a reweighting technique that does not depend on a Monte Carlo acceptance correction. 


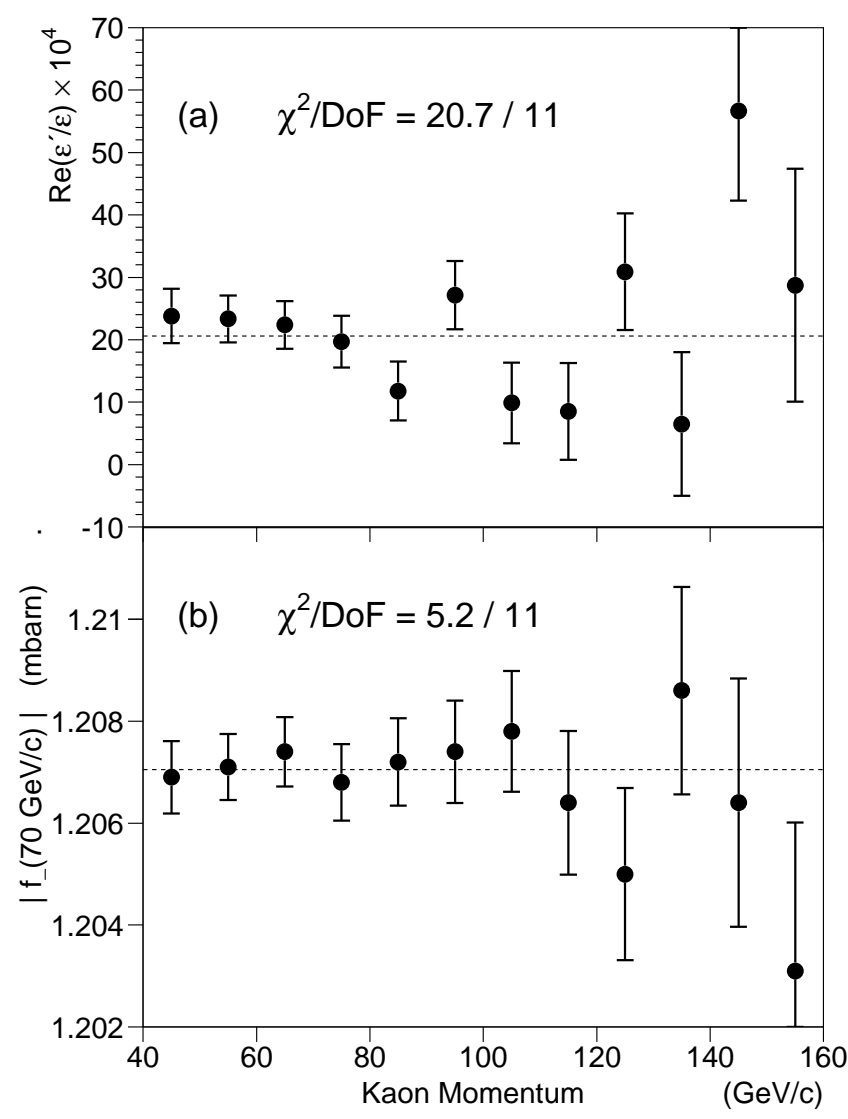

FIG. 26: Results of twelve independent fits in $10 \mathrm{GeV} / c$ wide $p$ bins for: (a) $\operatorname{Re}\left(\epsilon^{\prime} / \epsilon\right)$ and (b) $\left|f_{-}(70 \mathrm{GeV} / c)\right|$. Within each kaon momentum bin, $\operatorname{Re}\left(\epsilon^{\prime} / \epsilon\right)$ and $\left|f_{-}(70 \mathrm{GeV} / c)\right|$ are extracted from the same fit. Only statistical errors are shown. Each dashed line shows the average of all momentum bins.

This technique is similar to that used by the NA48 experiment [21. The "local acceptance" of $K \rightarrow \pi \pi$ decays in each $p-z$ bin $(1 \mathrm{GeV} / c \times 2 \mathrm{~m})$ is nearly identical in both beams, with the only difference arising from the effects of accidental activity. In this method, a weight is applied to vacuum beam events such that the regenerator and weighted vacuum beam events have the same statistical sampling of decay vertex and kaon energy. With the same local acceptance in the two beams as a function of $p$ and $z$, an ideal weight function eliminates differences in the reconstruction efficiencies and resolutions for $K \rightarrow \pi \pi$ in the two beams. The weight factor, which is applied eventby-event, is the a priori ratio of the regenerator beam and vacuum beam decay rates,

$$
W(p, z)=\frac{d \Gamma_{r e g} / d t(p, z)}{d \Gamma_{v a c} / d t(p, z)} .
$$

The functions $d \Gamma_{v a c} / d t$ and $d \Gamma_{r e g} / d t$ are similar to those given by Equations 13 and 14, respectively, with the modification that they are constrained to vanish upstream of the regenerator.

The $z$ distribution in each beam, without the weight factor, is shown in Fig. 21a-b, and the pion track
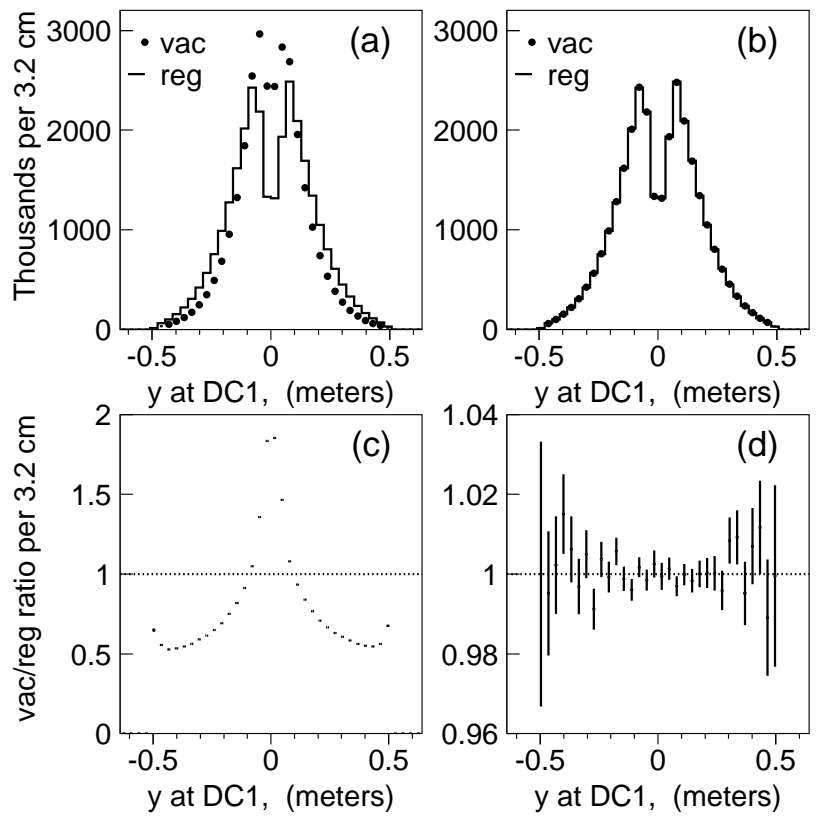

FIG. 27: (a) $K \rightarrow \pi^{+} \pi^{-}$track illumination in the DC1 $y$ view for vacuum (dots) and regenerator (histogram) beams. (b) The same track illumination after applying the weight factor to the vacuum beam. The vacuum-to-regenerator beam ratios are shown for each case in (c) and (d); note the different vertical scales for the ratios.

$y$-illumination at the first drift chamber is shown in Fig. 27a. The differences between the vacuum and regenerator beam distributions are due to the average acceptance difference coming from the different $K_{L}$ and $K_{S}$ lifetimes. The effect of $W(p, z)$ is that the vacuum beam distributions match those in the regenerator beam (Fig. 27b). The main drawback to this reweighting method is that the statistical uncertainty is increased by a factor of 1.7 compared to the standard analysis, because of the loss of vacuum beam events through the reweighting function. In addition, the reweighting technique is more sensitive to the neutral energy reconstruction because the weight factor (Eq. 23) depends on kaon energy.

The event reconstruction and selection are very similar to that of the standard analysis. In the $K \rightarrow \pi^{0} \pi^{0}$ mode, the event selection cuts are identical. The most significant difference in the reweighting analysis is the energy scale correction, where the absolute energy scale is corrected as opposed to the relative data-MC scale. The energy scale correction is derived from the difference between the $\gamma \gamma$ and $\pi^{+} \pi^{-} z$-vertex reconstructed in $K_{L} \rightarrow \pi^{+} \pi^{-} \pi^{0}$.

For the $K \rightarrow \pi^{+} \pi^{-}$selection, the reweighting analysis differs from the standard analysis by adding a center-ofenergy "RING" cut (Eq. 10). This cut is the same for neutral and charged events, and eliminates the need to correct for the effect of kaon scattering in the movable absorber upstream of the regenerator (Fig. 1). 
$R e\left(\epsilon^{\prime} / \epsilon\right)$ is extracted in a fit which compares the background-subtracted yields to a prediction function. The prediction function is the same as Eqs. 13-14, except that in the vacuum beam, both the data and the prediction function include the weight factor. As in the standard fit, there are 48 measured inputs, which correspond to the numbers of $K \rightarrow \pi \pi$ events in each $10 \mathrm{GeV} / c$ wide kaon momentum bin, for both decay modes and both regenerator positions. The free parameters in the fit include 24 kaon fluxes, the regeneration amplitude and power-law slope (Eq. 17), and $\operatorname{Re}\left(\epsilon^{\prime} / \epsilon\right)$. In the reweighting fit, the two regeneration parameters also appear in the vacuum beam fit functional via the reweighting function, where they are not varied. We find that the value of $\operatorname{Re}\left(\epsilon^{\prime} / \epsilon\right)$ is quite insensitive to the regeneration parameters used in the reweighting function. As in the standard $\operatorname{Re}\left(\epsilon^{\prime} / \epsilon\right)$ fit, $\Delta m$ and $\tau_{S}$ are fixed to the values from the measurements in Sec. VIA. There are a total of 27 free parameters and 21 degrees of freedom in the reweighting method fit.

The systematic errors for the reweighting analysis are shown in Table IX. In the neutral decay mode, the largest systematic uncertainty results from the sensitivity to the minimum photon cluster energy. The other large source of systematic uncertainty results from accidental activity. The level of accidental activity in the detector is slightly different for the two beams; this affects the local acceptance differently in the vacuum and regenerator beams and is not accounted for by the weight factor. In the standard analysis, accidental effects are accounted for in the Monte Carlo acceptance correction.

To determine the uncorrelated statistical uncertainty between the standard and reweighting analyses, a large number of Monte Carlo samples are generated and fit with both methods; the uncorrelated uncertainty results mainly from the effective loss in statistics in the reweighting method. The uncorrelated systematic error is mainly from the uncertainty in the minimum cluster energy requirement in the reweighting analysis; there are also contributions from the acceptance correction in the standard analysis and accidental effects in the reweighting analysis.

The reweighting and standard analyses were both applied to the part of the 1997 data sample that was not used in the previous publication, corresponding to roughly $3 / 4$ of the total sample. There is good agreement between the two analyses: the difference between the reweighting and standard $\operatorname{Re}\left(\epsilon^{\prime} / \epsilon\right)$ results is

$$
\begin{aligned}
\Delta\left[\operatorname{Re}\left(\epsilon^{\prime} / \epsilon\right)\right] & =[+1.5 \pm 2.1(\text { stat }) \pm 3.3(\text { syst })] \times 10^{-4} \\
& =(+1.5 \pm 3.9) \times 10^{-4}
\end{aligned}
$$

where the errors reflect the uncorrelated uncertainty between the two methods.
TABLE IX: Systematic Uncertainties in $\operatorname{Re}\left(\epsilon^{\prime} / \epsilon\right)$ from the reweighting analysis.

\begin{tabular}{l|l|c}
\hline \hline & Source of & $\begin{array}{c}\operatorname{Re}\left(\epsilon^{\prime} / \epsilon\right) \text { Uncertainty } \\
\left(\times 10^{-4}\right)\end{array}$ \\
\hline Sample & Uncertainty & 1.31 \\
& Backgrounds & 2.93 \\
& Reconstruction & 0.41 \\
& Trigger & 1.46 \\
\hline Charged & Accidental Bias & 0.16 \\
& Backgrounds & 0.87 \\
& Reconstruction & 1.09 \\
& Trigger & 0.81 \\
& Accidental Bias & 0.20 \\
& Regenerator location & 0.70 \\
\hline Common & Ring Number cut & 0.30 \\
& Reweighting Parameters & 3.98 \\
\hline Total & &
\end{tabular}

\section{MEASUREMENTS OF KAON PARAMETERS}

The regenerator beam decay distribution allows measurements of the kaon parameters $\tau_{S}$ and $\Delta m$, and CPT tests based on measurements of $\phi_{+-}$and $\Delta \phi$. The main difference compared to the $\operatorname{Re}\left(\epsilon^{\prime} / \epsilon\right)$ fit is that we fit the shape of decay distribution instead of the integrated yield. All of the fits discussed below use 2 meter wide $z$-bins in the regenerator beam from $124 \mathrm{~m}$ to $158 \mathrm{~m}$. In the vacuum beam, one $z$-bin from $110 \mathrm{~m}$ to $158 \mathrm{~m}$ is used to determine the kaon flux in each $10 \mathrm{GeV} / c$ momentum bin.

A $z$-binned fit increases the sensitivity to migrations in $z$. To allow for such migrations near the regenerator edge, we include an extra " $z$-shift" parameter which is the shift in the effective regenerator edge relative to the nominal value calculated in Section II C 3. In all fits, the charged and neutral data are consistent with no $z$-shift at the regenerator edge.

Systematic errors for the kaon parameter measurements are evaluated in a manner similar to the $\operatorname{Re}\left(\epsilon^{\prime} / \epsilon\right)$ analysis, and are summarized in Table $\mathrm{X}$. The sensitivity to $z$ migration is most pronounced in the $\Delta \phi$ uncertainty related to CsI energy reconstruction. The complicated $z$ dependence of the regenerator scattering background (Fig. 20) also contributes significant uncertainties to results obtained from $z$-binned fits.

\section{A. Measurement of $\Delta m$ and $\tau_{S}$}

To measure $\Delta m$ and $\tau_{S}$, we fit the charged and neutral modes separately and then combine results according to the statistical and uncorrelated systematic errors. We assume CPT symmetry (Eq. 15) by dynamically setting the value of $\phi_{\eta}$ equal to the superweak phase using the floated values of $\Delta m$ and $\tau_{S}$. The fit values of $\tau_{S}$ and $\Delta m$ are independent of the value of $\operatorname{Re}\left(\epsilon^{\prime} / \epsilon\right)$. 
TABLE X: Systematic uncertainties in $\Delta m, \tau_{S}, \phi_{+-}$, and $\Delta \phi$. " $\sigma_{\text {syst }}$ " refers to systematic uncertainty. The uncertainty associated with fitting is common to both decay modes. The "combined total" uncertainty is explained in the text.

\begin{tabular}{|c|c|c|c|c|}
\hline \multirow[b]{2}{*}{$\begin{array}{l}\text { Source of } \\
\text { Uncertainty }\end{array}$} & \multicolumn{4}{|c|}{$\sigma_{\text {syst }}$ for: } \\
\hline & $\begin{array}{c}\Delta m \\
\left(\times 10^{6} \hbar / s\right)\end{array}$ & $\begin{array}{c}\tau_{S} \\
\left(\times 10^{-12} \mathrm{~s}\right)\end{array}$ & $\begin{array}{l}\phi_{+-} \\
\left({ }^{\circ}\right)\end{array}$ & $\begin{array}{l}\Delta \phi \\
\left({ }^{\circ}\right)\end{array}$ \\
\hline \multicolumn{5}{|c|}{$K \rightarrow \pi^{+} \pi^{-}$Analysis: } \\
\hline Trigger & 0.2 & 0.004 & 0.10 & 0.02 \\
\hline Track reconstruction & 0.6 & 0.032 & 0.02 & 0.02 \\
\hline Selection efficiency & 3.2 & 0.011 & 0.35 & 0.06 \\
\hline Apertures & 2.8 & 0.038 & 0.76 & 0.09 \\
\hline Background & 0.8 & 0.002 & 0.01 & 0.01 \\
\hline Acceptance & 1.2 & 0.026 & 0.14 & 0.06 \\
\hline MC statistics & 2.6 & 0.012 & 0.28 & 0.05 \\
\hline Fitting & 13.3 & 0.045 & 0.80 & 0.03 \\
\hline$K \rightarrow \pi^{+} \pi^{-}$Total & 14.3 & 0.074 & 1.20 & 0.14 \\
\hline \multicolumn{5}{|c|}{$\bar{~} K \rightarrow \pi^{0} \pi^{0}$ Analysis: } \\
\hline Trigger & 0.4 & 0.013 & - & 0.03 \\
\hline CsI reconstruction & 8.1 & 0.094 & - & 0.37 \\
\hline Selection efficiency & 5.0 & 0.035 & - & 0.06 \\
\hline Apertures & 2.2 & 0.040 & - & 0.14 \\
\hline Background & 7.0 & 0.030 & - & 0.14 \\
\hline Acceptance & 2.0 & 0.030 & - & 0.05 \\
\hline MC statistics & 3.3 & 0.016 & - & 0.06 \\
\hline Fitting & 13.3 & 0.045 & - & 0.03 \\
\hline$K \rightarrow \pi^{0} \pi^{0}$ Total & 18.3 & 0.126 & - & 0.43 \\
\hline Combined Total & 14.2 & 0.069 & - & 0.50 \\
\hline
\end{tabular}

TABLE XI: $\Delta m$ and $\tau_{S}$ results for the regenerator beam charged and neutral data samples. The first uncertainty is statistical; the second is systematic.

\begin{tabular}{l|c|c|c}
\hline \hline $\begin{array}{c}\text { decay } \\
\text { mode }\end{array}$ & $\begin{array}{c}\Delta m \\
\left(\times 10^{6} \hbar_{\mathrm{s}}^{-1}\right)\end{array}$ & $\begin{array}{c}\tau_{S} \\
\left(\times 10^{-12} \mathrm{~s}\right)\end{array}$ & $\chi^{2} /$ dof \\
\hline$\pi^{+} \pi^{-}$ & $5266.7 \pm 5.9 \pm 14.3$ & $89.650 \pm 0.028 \pm 0.074$ & $228 / 199$ \\
$\pi^{0} \pi^{0}$ & $5237.3 \pm 10.6 \pm 18.3$ & $89.637 \pm 0.050 \pm 0.126$ & $195 / 199$ \\
\hline \hline
\end{tabular}

For each charged and neutral mode fit, there are 216 measured input quantities. The number of vacuum beam decays in each $10 \mathrm{GeV} / c$ momentum bin gives 12 inputs; the number of regenerator beam decays in each $2 \mathrm{~m} \times 10 \mathrm{GeV} / c$ bin adds $17 \times 12=204$ inputs. The floated parameters include the kaon flux in each of 12 momentum bins, the magnitude and phase of the regeneration amplitude, a $z$-shift parameter, $\Delta m$, and $\tau_{S}$; these 17 floated parameters lead to 199 degrees of freedom. The results of separate fits to the charged and neutral mode data are shown in Table XI. The difference between the charged and neutral mode results, after accounting for the common systematic uncertainty described below, is $1.6 \sigma$ for $\Delta m$ and $0.1 \sigma$ for $\tau_{S}$.

Systematic errors arising from data analysis are larger in the neutral decay mode than in the charged mode, primarily because of larger background and uncertainties in the CsI energy reconstruction. The systematic uncer- tainties due to regeneration properties (screening, attenuation, and analyticity) are more significant than in the $\operatorname{Re}\left(\epsilon^{\prime} / \epsilon\right)$ analysis because there is no cancellation between charged and neutral mode data. These uncertainties in the regeneration properties are common to the charged and neutral mode fits, and are applied to the final result after averaging. The common systematic uncertainty is $13.3 \times 10^{6} \hbar \mathrm{s}^{-1}$ on $\Delta m$, and $0.045 \times 10^{-12} \mathrm{~s}$ on $\tau_{S}$.

We combine the charged and neutral mode results weighted by the statistical uncertainty and the independent parts of the systematic uncertainty. The results are

$$
\begin{aligned}
\Delta m & =(5261 \pm 15) \times 10^{6} \hbar \mathrm{s}^{-1}, \\
\tau_{S} & =(89.65 \pm 0.07) \times 10^{-12} \mathrm{~s},
\end{aligned}
$$

which correspond to a superweak phase of

$$
\phi_{S W}=(43.38 \pm 0.10)^{\circ} .
$$

\section{B. Measurement of $\phi_{+-}$and $\phi_{+-}-\phi_{S W}$}

The fit for $\phi_{+-}$is similar to the $\Delta m-\tau_{S}$ fit. The main difference is that we remove the CPT assumption (Eq. 15) and float $\phi_{+-}$in addition to $\Delta m$ and $\tau_{S}$. The fit is performed to $K \rightarrow \pi^{+} \pi^{-}$data only. Compared to the $\Delta m-\tau_{S}$ fit, we have the same number of measured inputs $(216)$ and one additional free parameter $\left(\phi_{+-}\right)$, for a total of $216-18=198$ degrees of freedom.

There is a large correlation among $\phi_{+-}, \Delta m$, and $\tau_{S}$, which is illustrated in Fig. 31 (Appendix D). The $\Delta m-\tau_{S}$ correlation is much stronger than in a fit using the CPT assumption, and therefore results in a larger statistical uncertainty on $\Delta m$ and $\tau_{S}$. The $\phi_{+-}$statistical uncertainty in our fit is 2.4 times larger than in a fit with a fixed value of $\tau_{S}$.

The $0.76^{\circ}$ systematic uncertainty from apertures (Table $\mathrm{X}$ ) is mainly from the cell separation cut at the drift chambers (Sec. III Q). Different screening models result in a $0.75^{\circ}$ uncertainty on $\phi_{+-}$(Table VII). The value of $\phi_{+-}$depends on the regeneration phase $\phi_{\rho}$; a $0.25^{\circ}$ uncertainty from the analyticity assumption leads to a $0.25^{\circ}$ error on $\phi_{+-}$. The total systematic uncertainty on $\phi_{+-}$is $1.20^{\circ}$.

The results of the fit are:

$$
\begin{aligned}
\phi_{+-} & =[44.12 \pm 0.72(\text { stat }) \pm 1.20(\text { syst })]^{\circ} \\
& =(44.12 \pm 1.40)^{\circ} \\
\Delta m & =[5288 \pm 23(\text { stat })] \times 10^{6} \hbar_{\mathrm{s}^{-1}} \\
\tau_{S} & =[89.58 \pm 0.08(\text { stat })] \times 10^{-12} \mathrm{~s} \\
\chi^{2} / \nu & =223.6 / 197 .
\end{aligned}
$$

Next, we fit the deviation from the superweak phase, $\phi_{+-}-\phi_{S W}$, which is a direct test of CPT symmetry. Compared to the $\phi_{+-}$fit shown above, the fit for $\phi_{+-}-\phi_{S W}$ results in slightly reduced statistical and systematic uncertainties because the value of $\phi_{S W}$ is computed dynamically using the floated values of $\Delta m$ and 
$\tau_{S}($ Eq. 15), and is less sensitive to the correlations. The result of this fit is

$$
\begin{aligned}
\phi_{+-}-\phi_{S W} & =[+0.61 \pm 0.62(\text { stat }) \pm 1.01(\text { syst })]^{\circ} \\
& =(+0.61 \pm 1.19)^{\circ},
\end{aligned}
$$

and the $\chi^{2}$ is the same as for the $\phi_{+-}$fit (Eq. 28).

\section{Measurement of $\Delta \phi$}

The measurement of $\Delta \phi$ is performed in a simultaneous fit to neutral and charged mode data. The number of measured inputs is 432 , which is simply twice the number used in the $\Delta m-\tau_{S}$ fits, since both charged and neutral modes are used in the same fit. The floated parameters include the charged and neutral kaon fluxes in each of 12 momentum bins $(12+12=24)$, the regeneration amplitude and phase, one $z$-shift term in charged and one in neutral, the real and imaginary parts of $\epsilon^{\prime} / \epsilon, \Delta m, \tau_{S}$, and $\phi_{+-}$; these 33 floated parameters lead to 399 degrees of freedom. Note that the fit uses $\operatorname{Im}\left(\epsilon^{\prime} / \epsilon\right)$ instead of $\Delta \phi$

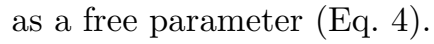

The fit for $\Delta \phi$ benefits from the cancellation of uncertainties in the regenerator properties. Also, there is little correlation with the other kaon parameters such as $\Delta m$, $\tau_{S}$, and the phase of $\epsilon$. Consequently, systematic uncertainties due to regenerator properties are small. The largest systematic uncertainty of $0.37^{\circ}$ is from the CsI cluster reconstruction in the neutral mode analysis.

There is a correlation between the real and imaginary parts of $\epsilon^{\prime} / \epsilon$, with a correlation coefficient of -0.565 . As a result, the statistical uncertainty for $\operatorname{Re}\left(\epsilon^{\prime} / \epsilon\right)$ is increased by about $20 \%$ compared to the standard fit that sets $\operatorname{Im}\left(\epsilon^{\prime} / \epsilon\right)=0$.

The results of the fit without CPT assumptions are

$$
\begin{aligned}
\operatorname{Im}\left(\epsilon^{\prime} / \epsilon\right) & =[-22.9 \pm 12.8(\text { stat }) \pm 26.2(\text { syst })] \times 10^{-4} \\
& =(-22.9 \pm 29.1) \times 10^{-4} \\
\operatorname{Re}\left(\epsilon^{\prime} / \epsilon\right) & =[+22.5 \pm 1.9(\text { stat })] \times 10^{-4} \\
\chi^{2} / \nu & =425 / 398 .
\end{aligned}
$$

In terms of $\Delta \phi$, the result is

$$
\begin{aligned}
\Delta \phi & =[+0.39 \pm 0.22(\text { stat }) \pm 0.45 \text { (syst) }]^{\circ} \\
& =(+0.39 \pm 0.50)^{\circ} .
\end{aligned}
$$

\section{Kaon Parameter Crosschecks}

The $K \rightarrow \pi \pi$ samples are divided into various subsets, among which we check the consistency of $\Delta m, \tau_{S}, \phi_{+-}$, and $\Delta \phi$. For all four measurements, we find good agreement between five month-long time periods, the two regenerator positions, and the two magnet polarities. The consistency of $\Delta m, \tau_{S}, \phi_{+-}$, and $\Delta \phi$ as a function of kaon momentum is shown in Fig. 28. There is good agreement among the 12 momentum bins in both the charged and neutral decay modes. Allowing each parameter to have a slope as a function of kaon momentum, the significance of each slope is between $0.5 \sigma$ and $1.5 \sigma$, consistent with no momentum dependence.

To check the dependence on proper decay time, the regenerator beam $K \rightarrow \pi \pi$ samples are divided into subsets with proper time less than and greater than $3 K_{S}$ lifetimes relative to the regenerator edge. This test is also sensitive to the significant background variations as a function of decay vertex (Fig. 20). The entire vacuum beam samples are used to determine the kaon flux in each $10 \mathrm{GeV} / c$ momentum bin, and the statistical uncertainty from the vacuum beam data is subtracted for these comparisons. For the sample with decays near the regenerator edge, $85 \%$ of the $K \rightarrow \pi \pi$ decay rate is from the $K_{S}$ term that is proportional to $|\rho|^{2}$ in Eq. 14; for the other sample, $42 \%$ of the $K \rightarrow \pi \pi$ decay rate is from the $K_{S}$ term. Fig. 29 shows consistent results between the two proper time ranges for the kaon parameter measurements. Note that the measurement of $\phi_{+-}$, which has strong correlations with $\Delta m$ and $\tau_{S}$, is more sensitive to early decay times; the measurement of $\Delta \phi$, which is very weakly correlated with $\Delta m$ and $\tau_{S}$, is more sensitive to later decay times.

\section{CONCLUSIONS}

In this paper, we report an improved measurement of direct $\mathrm{CP}$ violation in the decay of the neutral kaon:

$$
\begin{aligned}
R e\left(\epsilon^{\prime} / \epsilon\right) & =[20.7 \pm 1.48(\text { stat }) \pm 2.39(\text { syst })] \times 10^{-4} \\
& =(20.7 \pm 2.8) \times 10^{-4}
\end{aligned}
$$

This result, which supersedes reference [20], is consistent with the measurement from the NA48 collaboration [21, 40 $R e\left(\epsilon^{\prime} / \epsilon\right)=(15.3 \pm 2.6) \times 10^{-4}$. The average of our result and measurements from [18, 19, 21] gives $R e\left(\epsilon^{\prime} / \epsilon\right)=(17.2 \pm 1.8) \times 10^{-4}$ with a $13 \%$ confidence level.

In addition, we report new measurements of the $K_{L^{-}}$ $K_{S}$ mass difference and the $K_{S}$ lifetime:

$$
\begin{aligned}
& \Delta m=(5261 \pm 15) \times 10^{6} \hbar \mathrm{s}^{-1} \\
& \tau_{S}=(89.65 \pm 0.07) \times 10^{-12} \mathrm{~s}
\end{aligned}
$$

where CPT symmetry is assumed. Although these results are consistent with individual previous measurements used in the PDG averages 31], our results each differ from the PDG averages by more than two standard deviations. The $\mathrm{KTeV} \tau_{S}$ measurement is consistent with a recent NA48 measurement [4], and both results are much more precise than the PDG average.

Finally, we measure phase differences

$$
\begin{aligned}
\phi_{+-}-\phi_{S W} & =(+0.61 \pm 1.19)^{\circ} \\
\Delta \phi \equiv \phi_{00}-\phi_{+-} & =(+0.39 \pm 0.50)^{\circ}
\end{aligned}
$$



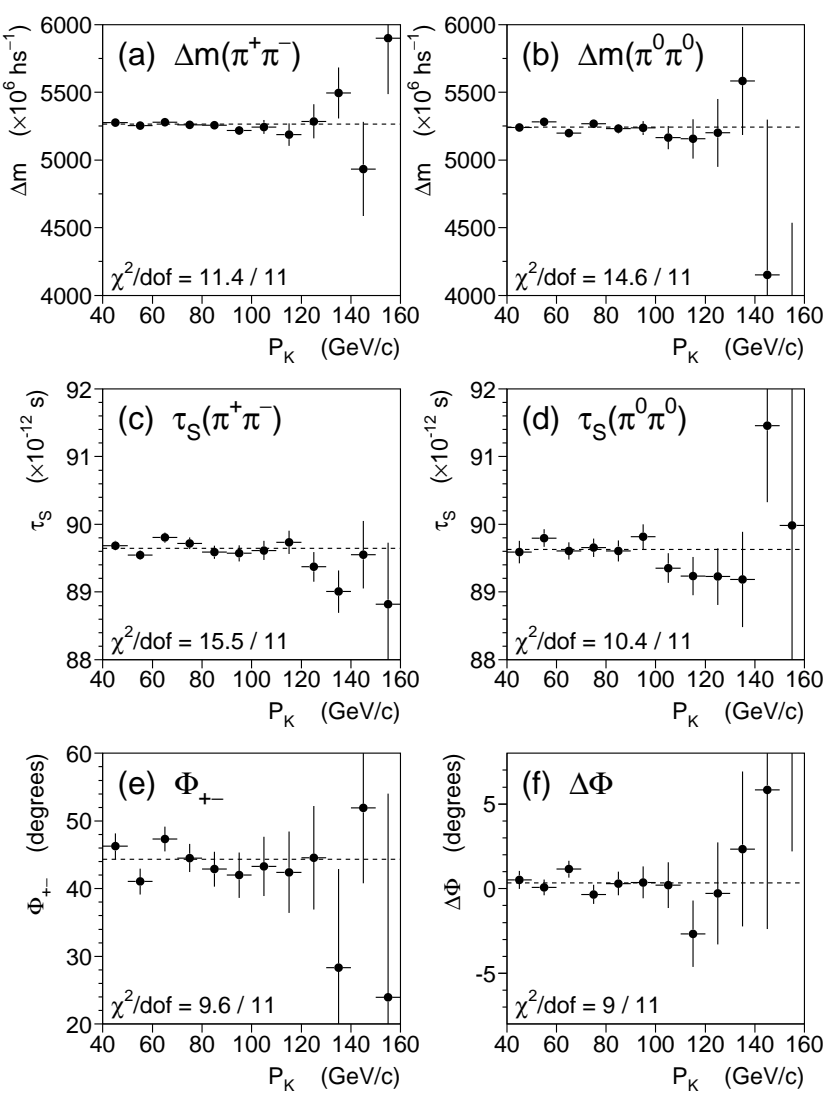

FIG. 28: Kaon parameters as a function of kaon momentum and decay mode are shown for: (a) $\Delta m$ from $\pi^{+} \pi^{-}$, (b) $\Delta m$ from $\pi^{0} \pi^{0}$, (c) $\tau_{S}$ from $\pi^{+} \pi^{-}$, (d) $\tau_{S}$ from $\pi^{0} \pi^{0}$, (e) $\phi_{+-}$, and (f) $\Delta \phi$. Each dashed horizontal line is the average of all momentum bins, and the $\chi^{2} /$ dof noted on each plot is for the consistency relative to the dashed line.

which are consistent with the CPT-symmetry prediction of zero. These phase differences are extracted from fits in which $\Delta m$ and $\tau_{S}$ are free parameters to avoid the CPT assumptions used to extract the nominal values in Eq. 32 . The $\Delta \phi$ result can be expressed in terms of $\operatorname{Im}\left(\epsilon^{\prime} / \epsilon\right)$ :

$$
\operatorname{Im}\left(\epsilon^{\prime} / \epsilon\right)=(-22.9 \pm 29.1) \times 10^{-4}
$$

\section{ACKNOWLEDGMENTS}

We gratefully acknowledge the support and effort of the Fermilab staff and the technical staffs of the participating institutions for their vital contributions. This work was supported in part by the U.S. Department of Energy, The National Science Foundation and The Ministry of Education and Science of Japan. In addition, A.R.B., E.B. and S.V.S. acknowledge support from the NYI program of the NSF; A.R.B. and E.B. from the Alfred P. Sloan Foundation; E.B. from the OJI program of the DOE; K.H., T.N. and M.S. from the Japan Society for the Promotion of Science; and R.F.B. from the Fundação
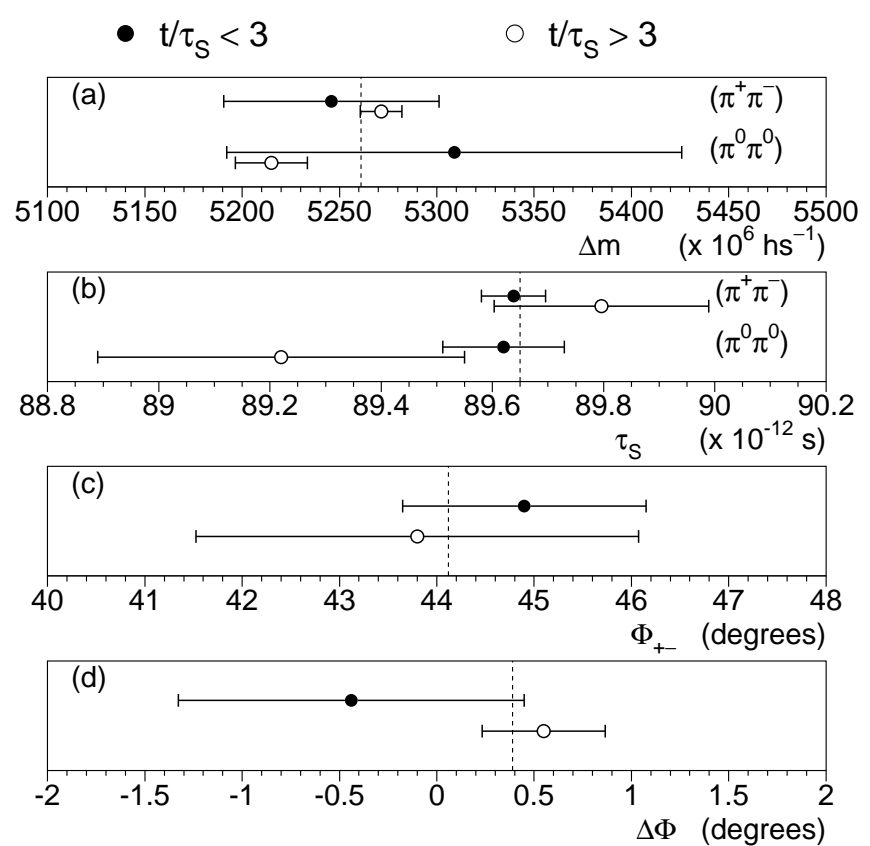

FIG. 29: Dots (open circles) show the kaon parameter measurements using regenerator beam data with proper time less (greater) than three $K_{S}$ lifetimes relative to the regenerator edge. For (a) $\Delta m$ and (b) $\tau_{S}$, the charged and neutral mode results are shown separately. For each pair of measurements (dot and open circle), the error bars reflect statistical uncertainties and are independent. Each vertical dashed line shows the nominal result using all of the data.

de Amparo à Pesquisa do Estado de São Paulo. P.S.S. acknowledges support from the Grainger Foundation.

\section{APPENDIX A: PRINCIPLES OF THE KTEV $R e\left(\epsilon^{\prime} / \epsilon\right)$ MEASUREMENT}

A simplified treatment of the $\mathrm{KTeV}$ measurement technique is presented here to illustrate some of the important cancellations that reduce systematic uncertainties in the $\operatorname{Re}\left(\epsilon^{\prime} / \epsilon\right)$ measurement. The measurement is based on the number of reconstructed $K \rightarrow \pi^{+} \pi^{-}$and $K \rightarrow \pi^{0} \pi^{0}$ decays in the vacuum and regenerator beams. To illustrate how these four quantities are related to $\operatorname{Re}\left(\epsilon^{\prime} / \epsilon\right)$, it is convenient to ignore the $K_{S}-K_{L}$ interference in both beams. In this simplified case, the four measured quantities are related to experimental parameters as follows:

$$
\begin{aligned}
N\left(\text { vac } \pi^{+} \pi^{-}\right) & \simeq \frac{\tau_{L}}{\tau_{S}} B_{S}^{+-} \mathcal{F}_{V}^{+-} \mathcal{A}_{V}^{+-}\left|\eta_{+-}\right|^{2} \frac{L}{\gamma c \tau_{L}}(\mathrm{~A} 1) \\
N\left(\text { reg } \pi^{+} \pi^{-}\right) & \simeq B_{S}^{+-} \mathcal{F}_{R}^{+-} \mathcal{A}_{R}^{+-}|\rho|^{2} T_{r e g} \quad(\mathrm{~A} 2) \\
N\left(\text { vac } \pi^{0} \pi^{0}\right) & \simeq \frac{\tau_{L}}{\tau_{S}} B_{S}^{00} \mathcal{F}_{V}^{00} \mathcal{A}_{V}^{00}\left|\eta_{00}\right|^{2} \frac{L}{\gamma c \tau_{L}} \\
N\left(\operatorname{reg} \pi^{0} \pi^{0}\right) & \simeq B_{S}^{00} \mathcal{F}_{R}^{00} \mathcal{A}_{R}^{00}|\rho|^{2} T_{r e g},
\end{aligned}
$$


where the $N$ 's are the observed number of $K \rightarrow \pi \pi$ decays in each beam, $B_{S}^{+-(00)}$ is the branching fraction of $K_{S} \rightarrow \pi^{+} \pi^{-}\left(\pi^{0} \pi^{0}\right), \mathcal{F}_{V}^{+-(00)}$ are the vacuum beam kaon fluxes, $\mathcal{F}_{R}^{+-(00)}$ are the kaon fluxes just upstream of the regenerator, the $\mathcal{A}$ 's are the acceptances determined by a Monte Carlo simulation, $L \sim 40 \mathrm{~m}$ is the length of the useful decay region, $T_{\text {reg }} \sim 0.18$ is the kaon flux transmission through the regenerator, $\gamma=E_{K} / M_{K} \sim 140$ is the average kaon boost, and $\rho \sim 0.03$ is the regeneration amplitude for forward scatters. The factor $L / \gamma c \tau_{S}$ is not present because essentially all of the $K_{S}$ decay within the decay region. The charged mode vacuum-to-regenerator single ratio is

$$
r_{+-}=\left|\frac{\eta_{+-}}{\rho}\right|^{2} \frac{\tau_{L}}{\tau_{S}} \cdot \frac{\mathcal{F}_{V}^{+-}}{\mathcal{F}_{R}^{+-}} \cdot \frac{\mathcal{A}_{V}^{+-}}{\mathcal{A}_{R}^{+-}} \cdot \frac{L}{\gamma c \tau_{L}} \cdot \frac{1}{T_{\text {reg }}}
$$

and similarly the neutral mode single ratio is obtained with +- replaced by 00 . To get a typical value of the single ratio, use $|\eta / \rho| \sim 0.07, \tau_{L} / \tau_{S}=580, \mathcal{F}_{V} / \mathcal{F}_{R} \simeq 2.32$ due to the movable absorber (Fig. 2), $\mathcal{A}_{V} / \mathcal{A}_{R} \sim 0.8$ and $L / \gamma c \tau_{L} \sim 0.02$; this gives $r \sim 0.6$, which shows that $\mathrm{KTeV}$ is designed to collect roughly the same statistics in the vacuum and regenerator beams. The statistical precision on $\operatorname{Re}\left(\epsilon^{\prime} / \epsilon\right)$ is limited by the number of vacuum beam $K \rightarrow \pi^{0} \pi^{0}$ decays; with $\mathcal{F}_{V}^{00} \simeq 2 \mathrm{MHz}$ and $\mathcal{A}_{V}^{00} \sim 0.05$, the rate of $K_{L} \rightarrow \pi^{0} \pi^{0}$ is $\sim 2 \mathrm{~Hz}$. The $5 \%$ acceptance used here is defined relative to all kaons and includes a livetime factor of 0.7 ; it is therefore smaller than the acceptance shown in Figure 22 that is defined within a specific momentum and $z$-vertex range.

The desired quantity, $\left|\eta_{+-} / \eta_{00}\right|^{2}$, is proportional to the experimentally measured double ratio, $r_{+-} / r_{00}$. The factors $\tau_{L} / \tau_{S}, T_{\text {reg }},|\rho|$, and $L / \gamma c \tau_{L}$ cancel in the double ratio. For the kaon flux cancellation, we use the constraint that the vacuum-to-regenerator kaon flux ratio is the same for both the charged and neutral decay modes,

$$
R_{F} \equiv \mathcal{F}_{V+-} / \mathcal{F}_{R+-}=\mathcal{F}_{V 00} / \mathcal{F}_{R 00} \simeq 2.32 \text {. }
$$

Note that Eq. A6 requires equal live-time for the vacuum and regenerator beams; the charged and neutral mode live-times, which are more difficult to control experimentally, do not have to be the same. If the beam collimation system results in small kaon-flux differences between the two neutral beams, switching the regenerator position between the two beams ensures that Eq. A6 is satisfied. The only quantities in the double ratio that do not cancel are the acceptances, for which we use a detailed Monte Carlo simulation.

The quantities $r_{+-}$and $r_{00}$ can be measured at different times provided that the following are the same for both measurements: (i) the kaon transmission in both the movable absorber and regenerator, (ii) the regeneration amplitude $\rho$ (iii) the distance between the primary $\mathrm{BeO}$ target and regenerator.

When Equations A1 A4 are modified to account for $K_{S}-K_{L}$ interference, there is no algebraic solution for $\operatorname{Re}\left(\epsilon^{\prime} / \epsilon\right)$; results are extracted from a fit described in Section IV G.

\section{APPENDIX B: FUNCTION FOR KAON SCATTERING IN THE REGENERATOR}

This appendix describes the function used to model the $K \rightarrow \pi \pi$ decay distribution for kaons that scatter in the regenerator. The function depends on decay time, $p_{T}^{2}$, and kaon momentum, and is also used to separate diffractive and inelastic contributions. The functional form is

$$
N_{\text {regscat }} \propto \sum_{j=1}^{6} A_{j} e^{\alpha_{j} p_{t}^{2}}\left|\hat{\rho}_{j} e^{\Lambda_{S} t}+\eta e^{\Lambda_{L} t}\right|^{2}
$$

where $A_{j}, \alpha_{j},\left|\hat{\rho}_{j}\right|$ and $\phi_{\hat{\rho}_{j}}$ are the 24 fit parameters, $\Lambda_{S, L}=i m_{S, L}-\frac{1}{2} \Gamma_{S, L}$, and $t$ is the proper time of the decay. Note that each $\hat{\rho}_{j}$ is an independent regeneration amplitude for scattering. The terms in Eq. B1 can be roughly associated with the known properties of kaon scattering in carbon, hydrogen and lead. In addition to these 24 parameters, there are two extra parameters that describe the momentum dependence of the phase $\left(\phi_{\hat{\rho}_{j}}\right)$ and $p_{T}^{2}$ slope $\left(\alpha_{j}\right)$ associated with diffractive scattering from the lead at the downstream edge of the regenerator. Of the 26 parameters in Eq. B1, 8 are fixed based on previously measured properties of kaon scattering. An additional 12 parameters are used to float the momentum dependence of $N_{\text {regscat }}$ in $10 \mathrm{GeV} / c$ bins; the momentum dependence for scattered kaons varies by only a few percent compared to that of unscattered kaons. The total number of free parameters in the regenerator scattering function is $18+12=30$.

The $\alpha_{j}$ parameters, which describe the exponential $p_{T}^{2}$ dependence, are used to distinguish between inelastic and diffractive scattering. The term with the broadest $p_{T}^{2}$ distribution has $\alpha^{-1}=2.4 \times 10^{5} \mathrm{MeV}^{2} / c^{2}$, and is identified with inelastic scattering. The other terms have much steeper $p_{T}^{2}$ distributions, with $5000<\alpha^{-1}<$ $70000 \mathrm{MeV}^{2} / c^{2}$, and are associated with diffractive scattering. Figure 30 shows the diffractive and inelastic contributions to regenerator scattering.

After determining the shapes of the diffractive and inelastic scattering distributions, the next step is the absolute normalization of the background relative to the coherent $K \rightarrow \pi \pi$ signal. In the charged analysis, we define $N_{\text {scat }}^{+-}$to be the number of reconstructed $K \rightarrow \pi^{+} \pi^{-}$ events with $p_{T}^{2}>2500 \mathrm{MeV}^{2} / c^{2}$, and $N_{\text {coh }}^{+-}$to be the number of coherent events with $p_{T}^{2}<250 \mathrm{MeV}^{2} / c^{2}$ (Fig. 18). The scattering level is adjusted in the simulation so that the reconstructed $N_{\text {scat }}^{+-} / N_{\text {coh }}^{+-}$ratio is the same in the data and MC. Note that $N_{\text {scat }}^{+-}$in data is obtained after subtracting collimator scatters and semileptonic decays.

In the neutral mode analysis, we define similar quantities in the regenerator beam: $N_{\mathrm{scat}}^{00}$ is the number of reconstructed $K \rightarrow \pi^{0} \pi^{0}$ events with $300<$ RING $<$ $800 \mathrm{~cm}^{2}$ (after subtracting the other background components) and $N_{\mathrm{coh}}^{00}$ is the number with RING $<110 \mathrm{~cm}^{2}$ 


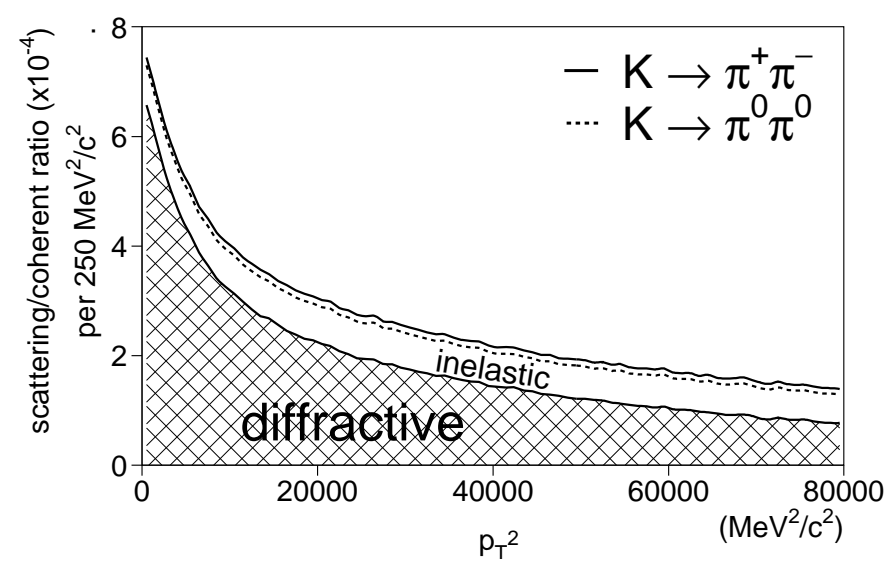

FIG. 30: Regenerator scattering $p_{T}^{2}$ distribution (solid curve) from a fit to acceptance-corrected $K \rightarrow \pi^{+} \pi^{-}$data. The normalization corresponds to the measured $0.074 \%$ scatteringto-coherent background level in the charged decay mode (Table III). The hatched (clear) region shows the diffractive (inelastic) component. The total (diffractive + inelastic) scattering-to-coherent ratio is shown for $K \rightarrow \pi^{+} \pi^{-}$(solid) and $K \rightarrow \pi^{0} \pi^{0}$ (dashed). The $3 \%$ charged-neutral difference reflects the additional $16 \%$ suppression of inelastic scattering in the neutral mode analysis.

(Fig. 19). Using the scattering-to-coherent ratio determined with acceptance-corrected $K \rightarrow \pi^{+} \pi^{-}$decays, the simulation over-predicts the $N_{\text {scat }}^{00} / N_{\text {coh }}^{00}$ ratio in data by $3 \%$; this difference results from the additional veto requirements in the neutral mode analysis, and is illustrated in Fig. 30. To match the $N_{\text {scat }}^{00} / N_{\text {coh }}^{00}$ ratio in data, the neutral mode scattering simulation is adjusted by a $16 \%$ reduction in the inelastic scattering level; note that the diffractive scattering level is not affected by the veto cuts, and is therefore the same in the charged and neutral analyses.

If we ignore the difference between diffractive and inelastic scattering, the simulated $N_{\text {scat }}^{00} / N_{\text {coh }}^{00}$ ratio can be adjusted by a $3 \%$ reduction in the total scattering level. Compared with the nominal $16 \%$ reduction in the inelastic level, a global 3\% adjustment gives equally good data-MC agreement in the RING distribution from 300 to $800 \mathrm{~cm}^{2}$ because the diffractive and inelastic distributions are very similar for $p_{T}^{2}>30000 \mathrm{MeV}^{2} / c^{2}$. In the coherent signal region, however, the diffractive and inelastic scattering distributions are different, leading to a $0.01 \%$ difference in the background prediction between these two ways of adjusting of the $N_{\text {scat }}^{00} / N_{\text {coh }}^{00}$ ratio. This difference is used in evaluating the systematic uncertainty on $\operatorname{Re}\left(\epsilon^{\prime} / \epsilon\right)$ in Section IIIF 5 .

\section{APPENDIX C: DISCUSSION OF THE PREVIOUS KTEV MEASUREMENT OF $R e\left(\epsilon^{\prime} / \epsilon\right)$}

The $\operatorname{Re}\left(\epsilon^{\prime} / \epsilon\right)$ result reported in Section $\mathrm{VA}$ includes a full reanalysis of our previously published data sample
[20]. The reanalysis of that data sample gives

$$
\begin{aligned}
\operatorname{Re}\left(\epsilon^{\prime} / \epsilon\right)= & {[23.2 \pm 3.0(\text { stat }) \pm 2.9(\text { syst })] \times 10^{-4} } \\
= & {[23.2 \pm 4.4] \times 10^{-4} } \\
& (\text { Reference }[20] \text { sample })
\end{aligned}
$$

with $\chi^{2} / \operatorname{dof}=18.7 / 21$. The $\operatorname{Re}\left(\epsilon^{\prime} / \epsilon\right)$ shift is $-4.8 \times 10^{-4}$ relative to the previous result in [20], and is larger than the previous systematic uncertainty of $2.8 \times 10^{-4}$. The shifts in $\operatorname{Re}\left(\epsilon^{\prime} / \epsilon\right)$ resulting from changes in the analysis are summarized in Table XII. The change in the regenerator scattering background for $K \rightarrow \pi^{0} \pi^{0}$ is mainly from correcting an error in the kaon scattering function. The other shifts are due to improvements and are consistent with the systematic uncertainties assigned in [20]. All of the changes in Table XII are from independent sources which are described below.

TABLE XII: Sources of the $R e\left(\epsilon^{\prime} / \epsilon\right)$ shift between reference [20] and re-analysis of the same data sample. The systematic $\sigma^{\prime}$ s $\left(\sigma_{\text {syst }}\right)$ are from 20 .

\begin{tabular}{l|c|c}
\hline \hline $\begin{array}{l}\text { Source of } \\
\text { change }\end{array}$ & $\begin{array}{r}\operatorname{Re}\left(\epsilon^{\prime} / \epsilon\right) \text { shift } \\
\left(\times 10^{-4}\right)\end{array}$ & $\begin{array}{c}\text { Number } \\
\text { of } \sigma_{\text {syst }}\end{array}$ \\
\hline Regenerator scattering & -1.7 & 2.1 \\
Collimator scattering & -0.2 & 0.6 \\
Screening & -0.3 & 1.5 \\
Regenerator transmission & -0.3 & 1.5 \\
Regenerator edge & -0.4 & 1.6 \\
Neutral analysis & +0.1 & 0.1 \\
Mask Anti MC & +0.3 & 1.3 \\
Absorber scattering MC & -0.6 & 0.6 \\
KTeV $\Delta m$ and $\tau_{S}$ & -0.5 & $3.1^{a}$ \\
MC fluctuation & -1.1 & 1.0 \\
\hline Total & -4.8 & 1.7 \\
\hline \hline
\end{tabular}

${ }^{a}$ Reference 20 used PDG98 values and errors for $\Delta m$ and $\tau_{S}$.

- The regenerator scattering function includes two extra scattering terms (Eq. B1) and two extra parameters to simulate subtle momentum-dependent features.

- Collimator scattering (Sec. IIIF 2) is measured after subtracting semileptonic decays, and the simulation includes two extra parameters to better describe the $K_{L} p_{T}^{2}$-dependence.

- In the $R e\left(\epsilon^{\prime} / \epsilon\right)$ fit (Sec. IV Q), a nuclear screening correction is used.

- The energy dependence of the regenerator transmission is measured with four times more $K_{L} \rightarrow \pi^{+} \pi^{-} \pi^{0}$ data.

- The calculation of the effective regenerator edge in the neutral decay mode (Fig. [b) includes kaon decays upstream of the last regenerator-lead piece instead of considering only decays in the last piece of lead. The effective regenerator edge in charged 
mode is based on a better technique to measure the veto threshold.

- Additional neutral mode veto cuts on hits in the trigger hodoscope and drift chambers reduce sensitivity to the transverse energy cut on CsI clusters.

- The Mask Anti simulation allows kaons and photons to punch through the lead-scintillator outside the beam-hole regions (Fig. 8).

- The absorber scattering simulation is improved to match beam shapes in a special run without the regenerator, but with the movable absorber still in place.

- We use our measurements of $\Delta m$ and $\tau_{S}$ that each differ by more than $2 \sigma$ from the PDG averages [31.

The changes listed above account for $-3.7 \times 10^{-4}$ of the shift in $\operatorname{Re}\left(\epsilon^{\prime} / \epsilon\right)$. We attribute the remaining shift of $-1.1 \times 10^{-4}$ to a $1 \sigma$ fluctuation between the two independent MC samples used for the acceptance correction.

The largest systematic uncertainty in [20] was based on the $z$-dependence of the acceptance for $K_{L} \rightarrow \pi^{+} \pi^{-}$ decays. Unfortunately, this data-MC discrepancy still remains in the reanalysis.

\section{APPENDIX D: CORRELATIONS AMONG KAON PARAMETER MEASUREMENTS}

The determination of correlations among different kaon parameters, including correlations arising from systematic uncertainties, is based on the following $\chi^{2}$ :

$$
\begin{aligned}
\chi^{2}\left(X, \alpha_{i}\right) & =(X-\bar{X}) C^{-1}(X-\bar{X})+\sum_{i} \alpha_{i}^{2} \\
\bar{X} & =\overline{X_{0}}+\sum_{i} \beta_{i} \alpha_{i} .
\end{aligned}
$$

$X$ is the vector of the measured physics quantities from the fit (e.g., $\Delta m$ and $\left.\tau_{S}\right)$, and $C$ is the covariance matrix including statistical uncertainties for both data and MC. The $\alpha_{i}$ are fit parameters representing the number of " $\sigma$ " associated with each source of systematic uncertainty that causes correlations among the physics quantities $\left(\alpha_{i}=1\right.$ corresponds to $1 \sigma$ change). $\overline{X_{0}}$ is the vector of physics quantities obtained in the nominal fit with all $\alpha_{i}=0$, and $\beta_{i}$ are the changes in the central value of $X$ corresponding to a $1 \sigma$ change in the systematic source $i$.

The total uncertainty in a physics quantity obtained from the $\chi^{2}$ in Eq. $D 1$ is equivalent to adding statistical and systematic uncertainties in quadrature. The minimization of this $\chi^{2}$ accounts for correlations among the physics quantities for each source of systematic uncertainty. We ignore correlations among the different sources of systematic uncertainty; these correlations are reduced by the grouping of systematic uncertainties shown in Table $\mathrm{X}$.
The total uncertainties and correlation coefficients for all of the fits are given in Table XIII. Note that the statistical and systematic uncertainties are larger in the fits in which CPT symmetry is not assumed. Figure 31 shows $1 \sigma$ contours of statistical and total uncertainties for the measurements of $\Delta m, \tau_{S}$, and $\phi_{+-}$. For the $\Delta m-\tau_{S}$ fit (Fig. 31a), systematic uncertainties have a significant effect on the $\Delta m-\tau_{S}$ correlation. For the $\phi_{+-}$fit (Fig. 31 bd), systematic uncertainties have a very small effect on the correlations. Figure 32 shows the correlation between the real and imaginary parts of $\epsilon^{\prime} / \epsilon$ from the fit without CPT assumptions; systematic uncertainties have a small effect on the correlation.

TABLE XIII: Total uncertainties and correlation coefficients for the three fits. The fit parameters and CPT assumptions

\begin{tabular}{|c|c|c|}
\hline \multicolumn{3}{|c|}{ I. $\Delta m-\tau_{S}$ Fit with CPT Assumption: } \\
\hline & $\Delta m$ & $\tau_{S}$ \\
\hline Total Error & $15 \times 10^{6} \hbar \mathrm{s}^{-1}$ & $0.07 \times 10^{-12} \mathrm{~s}$ \\
\hline \multicolumn{3}{|c|}{ Correlation coefficients: } \\
\hline$\Delta m$ & 1 . & \\
\hline$\tau_{S}$ & -0.396 & 1. \\
\hline
\end{tabular}
are listed above each set of values.

II. $\Delta m-\tau_{S}-\phi_{+-}$Fit without CPT Assumption:

\begin{tabular}{lccc} 
& $\Delta m$ & $\tau_{S}$ & $\phi_{+-}$ \\
\hline Total Error & $42 \times 10^{6} \hbar \mathrm{s}^{-1}$ & $0.13 \times 10^{-12} \mathrm{~s}$ & $1.40^{\circ}$ \\
\hline Correlation coefficients: & & & \\
$\Delta m$ & 1. & & \\
$\tau_{S}$ & -0.874 & 1. & \\
$\phi_{+-}$ & +0.987 & -0.898 & 1. \\
\hline \hline
\end{tabular}

III. $\epsilon^{\prime} / \epsilon$ Fit without CPT Assumption:

\begin{tabular}{lcc} 
& $\operatorname{Re}\left(\epsilon^{\prime} / \epsilon\right)$ & $\operatorname{Im}\left(\epsilon^{\prime} / \epsilon\right)$ \\
\hline Total Error & $4.0 \times 10^{-4}$ & $29.1 \times 10^{-4}$ \\
\hline Correlation coefficients: & & \\
$\operatorname{Re}\left(\epsilon^{\prime} / \epsilon\right)$ & 1. & \\
$\operatorname{Im}\left(\epsilon^{\prime} / \epsilon\right)$ & -0.647 & 1. \\
\hline \hline
\end{tabular}



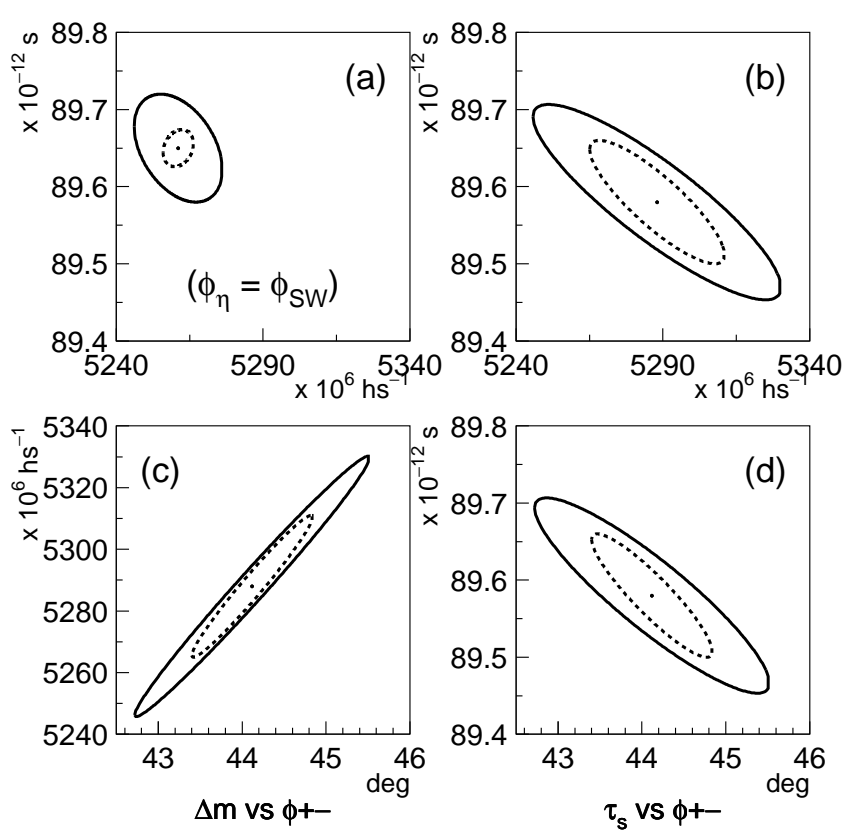

FIG. 31: $1 \sigma$ contours of statistical uncertainty (dashed) and total uncertainty (solid) for (a) $\Delta m-\tau_{S}$ fit (combined charged+neutral), and for (b)-(d) correlations from the $\phi_{+-}$ fit.

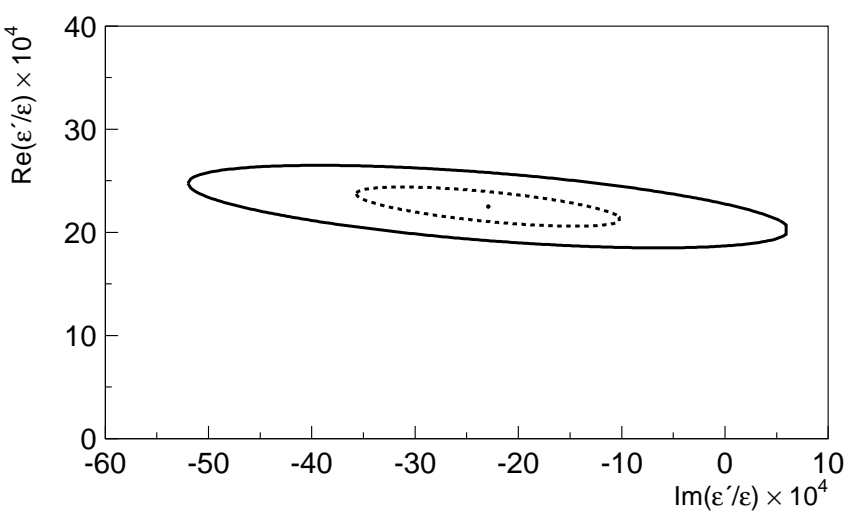

FIG. 32: $1 \sigma$ contours of statistical uncertainty (dashed) and total uncertainty (solid) for $\operatorname{Re}\left(\epsilon^{\prime} / \epsilon\right)$ vs. $\operatorname{Im}\left(\epsilon^{\prime} / \epsilon\right)$ from the fit with no CPT assumptions related to $\phi_{+-}$and $\phi_{00}$.
[1] J. H. Christenson, J. W. Cronin, V. L. Fitch, and R. Turlay, Phys. Rev. Lett. 13, 138 (1964).

[2] W. Ochs, $\pi N$ Newsletter 3, 25 (1991).

[3] M. Kobayashi and T. Maskawa, Prog. Theor. Phys. 49, 652 (1973).

[4] J. Ellis, M. K. Gaillard, and D. V. Nanopoulos, Nucl. Phys. B109, 213 (1976).

[5] F. J. Gilman and M. B. Wise, Phys. Lett. 83B, 83 (1979).

[6] T. Hambye et al., Nucl. Phys. B564, 391 (2000).

[7] H. Y. Cheng, Chin. J. Phys. 38, 1044 (2000).

[8] J. Bijnens and J. Prades, JHEP 06, 035 (2000).

[9] A. P. E. Pallante and I. Scimemi, Nucl. Phys. B617, 441 (2001).

[10] Y. Wu, Phys. Rev. D64, 016001 (2001).

[11] A. J. Buras et al., Nucl. Phys. B592, 55 (2001).

[12] S. Bertolini, J. O. Eeg, and M. Fabbrichesi, Phys. Rev. D63, 056009 (2001), hep-ph/0002234.

[13] S. Narison, Nucl. Phys. B593, 3 (2001).

[14] M. Ciuchini and G.Martinelli, Nucl. Phys. Proc. Suppl. 99B, 27 (2001).

[15] S. Aoki et al., Nucl. Phys. Proc. Suppl. 106, 332 (2002), (CP-PACS Collaboration).

[16] T. Blum et al., Nucl. Phys. Proc. Suppl. 106, 317 (2002), (RBC Collaboration).

[17] L. Wolfenstein, Phys. Rev. Lett. 13, 562 (1964).

[18] L. K. Gibbons et al., Phys. Rev. Lett. 70, 1203 (1993).

[19] G. D. Barr et al., Phys. Lett. B317, 233 (1993).

[20] A. Alavi-Harati et al., Phys. Rev. Lett. 83, 22 (1999).

[21] A. Lai et al. (NA48), Eur. Phys. J. C 22, 231 (2001).

[22] J. Graham, Ph.D thesis, The University of Chicago (2001); V. Prasad, Ph.D thesis, The University of
Chicago (2002); P. S. Shawhan, Ph.D thesis, The University of Chicago (1999).

[23] A. Roodman, in Proceedings of the Seventh International Conference on Calorimetry in High Energy Physics, edited by E. Cheu et al. (World Scientific, 1998), p. 89.

[24] J. Whitmore, Nucl. Instrum. Methods Phys. Res., Sect. A 409, 687 (1998).

[25] M. L. Good, Phys. Rev. 121, 311 (1961).

[26] P. S. Shawhan, Ph.D. thesis, The University of Chicago (1999).

[27] C. Bown et al., Nucl. Instrum. Methods Phys. Res., Sect. A 369, 248 (1996).

[28] A. J. Malensek, Fermilab Report FN-341 (errata), 1981.

[29] A. Alavi-Harati et al., Phys. Rev. Lett. 86, 761 (2001).

[30] R. Brun et al. (1994), GEANT 3.21, CERN, Geneva.

[31] Particle Data Group, Eur. Phys. J. C C15, 1 (2000).

[32] M. Woods, Ph.D. thesis, The University of Chicago (1988).

[33] F. James and M. Roos (1994), Minuit, CERN, Geneva.

[34] L. K. Gibbons et al., Phys. Rev. D 55, 6625 (1997).

[35] F. Gilman, Phys. Rev. 171, 1453 (1968).

[36] R. J. Glauber, Phys. Rev. 100, 242 (1955).

[37] V. Franco and R. J. Glauber, Phys. Rev. 142, 1195 (1966).

[38] L. Bertocchi and D. Treleani, Nuovo Cim. 50A, 338 (1979).

[39] R. A. Briere and B. Winstein, Phys. Rev. Lett. 75, 402 (1995).

[40] Using additional data collected in 2001, the NA48 collaboration recently presented an improved measurement of $\operatorname{Re}\left(\epsilon^{\prime} / \epsilon\right)=(14.7 \pm 2.2) \times 10^{-4}$ (C. Lazzeroni, XIVth 
Rencontres de Blois, France, 2002).

[41] A. Lai et al. (NA48) (2002), accepted by Phys. Lett. B; preprint CERN-EP/2002-028.

[42] Approximate expressions for the regeneration amplitude resulting from fits to $\mathrm{KTeV}$ data are $|\rho| \simeq 0.03(p / 70)^{-0.54}$ and $\phi_{\rho} \simeq\left[-34+12(p / 140-1)^{2}\right]^{\circ}$. 